\title{
Analytic descriptions for transitional nuclei near the critical point
}

\author{
M. A. Caprio* and F. Iachello \\ Center for Theoretical Physics, Sloane Physics Laboratory, Yale University, New \\ Haven, Connecticut 06520-8120, USA
}

\begin{abstract}
Exact solutions of the Bohr Hamiltonian with a five-dimensional square well potential, in isolation or coupled to a fermion by the five-dimensional spin-orbit interaction, are considered as examples of a new class of dynamical symmetry or Bose-Fermi dynamical symmetry. The solutions provide baselines for experimental studies of even-even $[\mathrm{E}(5)]$ and odd-mass $[\mathrm{E}(5 \mid 4)]$ nuclei near the critical point of the spherical to deformed $\gamma$-unstable phase transition.
\end{abstract}

PACS: 21.60.Ev, 21.10.Re, 21.60.Fw

\section{Introduction}

Dynamical symmetries have played an important role in the spectroscopy of nuclei, in particular in the description of their collective properties. A dynamical symmetry $[1,2]$ occurs when the Hamiltonian is constructed from the Casimir operators of the Lie algebras in a subalgebra chain $\left(G \supset G^{\prime} \supset\right.$ $\left.G^{\prime \prime} \supset \cdots\right)$. Dynamical symmetries yield good quantum numbers, labeling the irreducible representations of $G \supset G^{\prime} \supset G^{\prime \prime} \supset \cdots$, and make possible analytic solutions for the eigenvalues, eigenstates, and related observables. They thereby allow simple and straightforward comparison with experiment. Most applications of dynamical symmetries have been in the context of algebraic models, such as the interacting boson model (IBM) for nuclei [3]. The algebraic structure of this model is based upon $\mathrm{U}(6)$, and three dynamical symmetries arise, involving the subalgebras $\mathrm{U}(5), \mathrm{SO}(6)$, and $\mathrm{SU}(3)$. These symmetries can be related to the geometric description of nuclei [4-6], through the geometry

\footnotetext{
* Corresponding author.

Email address: mark. caprio@yale.edu (M. A. Caprio).
} 
$\mathrm{U}(6) /[\mathrm{U}(5) \otimes \mathrm{U}(1)]$ associated with $\mathrm{U}(6)[7-9]$. They are seen to describe limiting cases of geometric structure involving the quadrupole degree of freedom: spherical oscillator $[\mathrm{U}(5)], \gamma$-soft rotor $[\mathrm{SO}(6)]$, and axially symmetric rotor $[\mathrm{SU}(3)]$ structure. However, the intermediate situations between the structural limits are often of greatest interest, both for applications to actual transitional nuclei $[10,11]$ and in the study of phase transitions between the structural limits [12]. For these situations, results can usually only be obtained by numerical diagonalization of the Hamiltonian.

Recently, it has been suggested [13] that there is a class of dynamical symmetry which can be useful in the analysis of experimental data near the critical point of a phase transition. These symmetries cannot easily be formulated in algebraic models but arise naturally in models based upon the consideration of differential equations, such as the geometric description of nuclei with the Bohr Hamiltonian [4-6]. These symmetries are defined as dynamical symmetries in the traditional sense $[1,2]$, in that the Hamiltonian is written in terms of the Casimir operators of a chain of Lie algebras $G \supset G^{\prime} \supset G^{\prime \prime} \supset \cdots$, but the operators are now differential operators defined on a coordinate space restricted to a bounded domain.

A simple application of this approach is to the spectroscopy of nuclei near the critical point of the second order phase transition between the $\mathrm{U}(5)$ and $\mathrm{SO}(6)$ structural limits. The resulting description, denoted $\mathrm{E}(5)$, is simply the solution of the Bohr Hamiltonian for a five-dimensional square well potential [14], which is an idealized form of the potential near the critical point of the phase transition [13]. The first part of this article (after a brief summary of some prerequisite results for the five-dimensional square well potential) describes the algebraic properties of the E(5) dynamical symmetry and extends the calculations for electromagnetic matrix elements beyond the basic results reported in Ref. [13]. The calculations include electromagnetic operators involving higher order terms and make use of a recent construction of the angular wave functions (hyperspherical harmonics) in terms of orthogonalized products of generating functions $[15,16]$.

While dynamical symmetries in their simplest form involve purely bosonic or purely fermionic realizations of Lie algebras, dynamical symmetries can also be formulated for mixed bosonic and fermionic systems. Bose-Fermi dynamical symmetries [17] have mostly been applied within the framework of the interacting boson fermion model (IBFM) for nuclei [18]. For a nucleus consisting of a core coupled to a fermion distributed among orbitals with angular momenta $j$, the algebraic structure is based on the Lie algebra $\mathrm{U}_{B}(6) \otimes \mathrm{U}_{F}\left[\sum_{j}(2 j+1)\right]$, which can then be embedded in the superalgebra $\mathrm{U}\left[6 \mid \sum_{j}(2 j+1)\right]$ [19]. The dynamical Bose-Fermi symmetries of the IBFM have been very useful in the description of odd-mass nuclei, but the symmetries have only been applicable to nuclei at structural limits. For odd-mass nuclei, even more than for 
even-even nuclei, intermediate situations between the structural limits are of greatest interest, both for application to specific nuclei and for the study of the influence of coupled fermions on nuclear phase transitions.

In the second part of this article, the dynamical symmetry approach to describing structure near the critical point of a phase transition is extended to the odd-mass Bose-Fermi system. The simplest case of such a Bose-Fermi dynamical symmetry arises for a particle with $j=3 / 2$ coupled to the quadrupole degrees of freedom by a five dimensional spin-orbit interaction, leading to an $\mathrm{E}(5) \otimes \mathrm{U}(4)$ algebraic structure. Here we discuss the solution of the Bohr Hamiltonian with a square well potential in the presence of such a coupling. The calculation of electromagnetic observables is considered in detail. Preliminary results were reported in Ref. [20], where the notation $\mathrm{E}(5 \mid 4)$ was adopted for the model. A byproduct of the calculation is a systematic method for construction of the necessary $\operatorname{Spin}(5) \supset \operatorname{Spin}(3)$ isoscalar factors, relevant not only to the situation discussed here but to all problems involving Spin(5) coupling schemes.

The E(5) model has provided the basis for both experimental investigations and further theoretical developments [21-30]. Preliminary experimental and theoretical studies involving $\mathrm{E}(5 \mid 4)$ have also been carried out $[31,32]$. This article is meant primarily to provide a baseline for further study, both experimental and theoretical, of nuclei near the critical point of the spherical to deformed $\gamma$-unstable phase transition.

\section{Transitional symmetry for the $\gamma$-independent potential}

\subsection{Hamiltonian and solution}

We begin by considering an analytic solution describing the structure of even-even nuclei near the critical point of the transition between spherical and deformed $\gamma$-soft structure. The resulting $\mathrm{E}(5)$ model provides a benchmark for the basic features of nuclear structure soft to both $\gamma$ and $\beta$ deformation. It was presented in part in Ref. [13]. We consider it here in further detail both since it provides a basis for comparison with experimental data (e.g., Refs. [21-23, 25, 26]) and since it provides the foundation for the description of transitional odd-mass nuclei considered in Sec. 3.

For this analysis, we consider the Bohr Hamiltonian [4-6],

$$
H=\frac{\hbar^{2}}{2 B} \tilde{\pi} \cdot \tilde{\pi}+V(\alpha)
$$


Here $\alpha_{\mu}(\mu=0, \pm 1, \pm 2)$ are the quadrupole deformation coordinates, $\pi_{\mu}$ are the conjugate momenta, and standard spherical tensor notation [33] is used, with $\tilde{T}_{\mu}^{(\lambda)} \equiv(-)^{\lambda-\mu} T_{-\mu}^{(\lambda)}$. The quadrupole coordinates may be written in terms of the intrinsic deformation variables $\beta$ and $\gamma$ and the Euler angles $\left(\vartheta_{1}, \vartheta_{2}, \vartheta_{3}\right)$ as

$$
\alpha_{\mu}=\beta\left[\mathscr{D}_{\mu 0}^{(2)}(\vartheta) \cos \gamma+\frac{1}{\sqrt{2}}\left[\mathscr{D}_{\mu 2}^{(2)}(\vartheta)+\mathscr{D}_{\mu-2}^{(2)}(\vartheta)\right] \sin \gamma\right] .
$$

The Hamiltonian, reexpressed in terms of these variables, is

$$
\begin{array}{r}
H=-\frac{\hbar^{2}}{2 B}\left[\frac{1}{\beta^{4}} \frac{\partial}{\partial \beta} \beta^{4} \frac{\partial}{\partial \beta}+\frac{1}{\beta^{2}}\left(\frac{1}{\sin 3 \gamma} \frac{\partial}{\partial \gamma} \sin 3 \gamma \frac{\partial}{\partial \gamma}-\frac{1}{4} \sum_{\kappa} \frac{\hat{L}_{\kappa}^{\prime 2}}{\sin ^{2}\left(\gamma-\frac{2}{3} \pi \kappa\right)}\right)\right] \\
+V(\beta, \gamma), \quad(2.3)
\end{array}
$$

where the $\hat{L}_{\kappa}^{\prime}$ are the intrinsic frame angular momentum operators. We consider here specifically the case in which the potential is $\gamma$-independent, i.e., $V(\beta, \gamma)=V(\beta)$. A $\gamma$-independent potential suffices to describe spherical oscillator structure, deformed $\gamma$-soft structure [14], and the transition between them. This range of structural possibilities corresponds to the $\mathrm{U}(5)-\mathrm{SO}(6)$ transition in the interacting boson model (IBM) [34].

The Hamiltonian (2.3) with $\gamma$-independent potential is invariant under rotations in the five-dimensional space of the coordinates $\alpha_{\mu}$. The resulting $\mathrm{SO}(5)$ symmetry yields the five-dimensional analogue of the central force problem. A separation of "radial" $(\beta)$ and "angular" ( $\gamma$ and $\vartheta)$ variables can be carried out in the standard way $[14,35]$. The eigenfunctions are of the form

$$
\Phi(\beta, \gamma, \vartheta)=f(\beta) \Psi(\gamma, \vartheta) .
$$

The angular and radial factors satisfy the equations

$$
\left[-\frac{1}{\sin 3 \gamma} \frac{\partial}{\partial \gamma} \sin 3 \gamma \frac{\partial}{\partial \gamma}+\frac{1}{4} \sum_{\kappa} \frac{\hat{L}_{\kappa}^{\prime 2}}{\sin ^{2}\left(\gamma-\frac{2}{3} \pi \kappa\right)}\right] \Psi(\gamma, \vartheta)=\Lambda \Psi(\gamma, \vartheta)
$$

and

$$
\left[-\frac{1}{\beta^{4}} \frac{\partial}{\partial \beta} \beta^{4} \frac{\partial}{\partial \beta}+\frac{\Lambda}{\beta^{2}}+V(\beta)\right] f(\beta)=\varepsilon f(\beta),
$$

related by the separation constant $\Lambda$. [Here, and for the remainder of this article, we set $\hbar^{2} /(2 B)=1$. This can always be achieved by transformation to the reduced eigenvalues $\varepsilon^{\prime}=2 B \varepsilon / \hbar^{2}$ and reduced potential $V^{\prime}(\beta)=2 B V(\beta) / \hbar^{2}$.]

The solutions for the "angular" wave functions, $\Psi_{\tau \tilde{\nu}_{\triangle} L M_{L}}(\gamma, \vartheta)$, are common to all $\gamma$-soft problems and are well known $[15,16,36]$. They can be con- 
structed explicitly as functions of the form

$$
\Psi_{\tau \tilde{\nu} \triangle L M_{L}}(\gamma, \vartheta)=\sum_{\substack{K=0 \\ \text { even }}}^{L} F_{\tau \tilde{\nu}_{\triangle} L K}(\gamma) \phi_{M_{L} K}^{L}(\vartheta),
$$

where $\phi_{M K}^{L}(\vartheta) \equiv\left[(2 L+1) /\left(16 \pi^{2}\left(1+\delta_{K}\right)\right)\right]^{1 / 2}\left[\mathscr{D}_{M K}^{(L)}(\vartheta)+(-)^{L} \mathscr{D}_{M-K}^{(L)}(\vartheta)\right]$ is a symmetrized, normalized combination of $\mathscr{D}$ functions, and the $F_{\tau \tilde{\nu} \triangle L K}(\gamma)$ are polynomials involving trigonometric functions of $\gamma[16]$. Their eigenvalues in (2.5) are $\Lambda=\tau(\tau+3)$, for $\tau=0,1, \ldots$ (The quantum numbers are defined more fully below.)

The radial equation (2.6) posesses analytic solutions (or dynamical symmetries) only for a limited set of potentials. It is equivalent to the usual one-dimensional Schrödinger equation with an effective radial potential term $\propto 1 / \beta^{2}$. This can easily be seen by transformation to the "auxiliary" radial wave function $\varphi(\beta) \equiv \beta^{-2} f(\beta)$, which satisfies

$$
\left[-\frac{\partial^{2}}{\partial \beta^{2}}+\frac{\Lambda+2}{\beta^{2}}+V(\beta)\right] \varphi(\beta)=\varepsilon \varphi(\beta) .
$$

This equation posesses analytic solutions [37] for only two classes of potential, the generalized oscillator potential

$$
V(\beta)=a \beta^{2}+b \beta^{-2},
$$

which has been used in a study of the $\mathrm{U}(5)-\mathrm{SO}(6)$ transition by Elliott, Evans, and Park [38], and the generalized Coulomb potential

$$
V(\beta)=a \beta^{-1}+b \beta^{-2}
$$

The free potential, $V(\beta)=0$, occurs as a limiting case $(a=b=0)$ of either potential (2.9) or (2.10). The free potential, though exceedingly simple, is of special interest for the description of structure near the critical point between spherical and deformed structure. A potential $V(\beta)$ which is flat with respect to $\beta$ allows the nucleus to assume either a spherical $(\beta=0)$ or deformed $(\beta>0)$ shape with minimal energy penalty. An idealized approximation near the critical point is thus the five-dimensional infinite square well potential [14],

$$
V(\beta)= \begin{cases}0 & \beta \leq \beta_{W} \\ \infty & \beta>\beta_{W}\end{cases}
$$

which is just the free potential on the bounded domain $\beta \leq \beta_{W}$. Solution proceeds exactly as for the classic three-dimensional spherical well problem [37]. The radial equation $(2.6)$ or $(2.8)$ is, for $V(\beta)=0$, equivalent to 
Table 1

Angular momentum contents of the symmetric representations $(\tau, 0)$ of $\mathrm{SO}(5)$, for $\tau \leq 6$.

\begin{tabular}{lllllllllll}
\hline$\tau$ & $L$ & & & & \\
\hline 0 & 0 & & & & & & & & & \\
1 & 2 & & & & & & & & & \\
2 & 4 & 2 & & & & & & & & \\
3 & 6 & 4 & 3 & 0 & & & & & & \\
4 & 8 & 6 & 5 & 4 & 2 & & & & & \\
5 & 10 & 8 & 7 & 6 & 5 & 4 & 2 & & & \\
6 & 12 & 10 & 9 & 8 & 7 & 6 & 6 & 4 & 3 & 0 \\
\hline
\end{tabular}

the Bessel equation (see Refs. [13,37] for details). It is satisfied by functions $f(\beta) \propto \beta^{-3 / 2} J_{\nu}\left(\varepsilon^{1 / 2} \beta\right)$, with Bessel function order

$$
\nu=(\Lambda+9 / 4)^{1 / 2}
$$

or, in terms of $\tau, \nu(\tau)=\tau+3 / 2$. The boundary condition arising from the well wall $\left[f\left(\beta_{W}\right)=0\right]$ restricts the eigenvalue to

$$
\varepsilon_{\xi \tau}=\left(\frac{x_{\nu(\tau), \xi}}{\beta_{W}}\right)^{2}
$$

where $x_{\nu, \xi}$ is the $\xi$ th zero of $J_{\nu}$ [39]. The resulting radial eigenfunctions are

$$
f_{\xi \tau}(\beta)=C_{\xi \tau} \beta^{-3 / 2} J_{\nu(\tau)}\left(\frac{x_{\nu(\tau), \xi} \beta}{\beta_{W}}\right) .
$$

The normalization integral with respect to the metric $\beta^{4} d \beta[5]$ can be evaluated explicitly [40], giving

$$
C_{\xi \tau}=\beta_{W}^{-5 / 2}\left[-J_{\nu(\tau)-1}\left(x_{\nu(\tau), \xi}\right) J_{\nu(\tau)+1}\left(x_{\nu(\tau), \xi}\right)\right]^{-1 / 2} .
$$

As usual for problems with $\mathrm{SO}(5)$ symmetry, each value of $\tau$ yields a multiplet of degenerate states of various angular momenta $L$, according to the $\mathrm{SO}(5) \supset \mathrm{SO}(3)$ branching relations $[16,35,41,42]$. The angular momentum contents of the lowest $\mathrm{SO}(5)$ representations $(\tau, 0)$ are summarized for convenience in Table 1 . For $\tau \geq 6$, the same angular momentum can occur more than once within an $\mathrm{SO}(5)$ representation, and an additional multiplicity index, here chosen as $\tilde{\nu}_{\triangle}[43]$, is needed to distinguish between angular wave functions with otherwise identical quantum numbers. The eigenstates of the E(5) Hamiltonian are fully specified by the angular quantum numbers $\left(\tau, \tilde{\nu}_{\triangle}\right.$, $L$, and $\left.M_{L}\right)$ together with the radial quantum number $\xi(\xi=1,2, \ldots)$. The eigenstates may thus be denoted $\left|\xi \tau \tilde{\nu}_{\triangle} L M_{L}\right\rangle$ or, more concisely, $L_{\xi, \tau}^{+}$as in Ref. [13]. The levels, quantum numbers, and excitation energies obtained for the E(5) model are summarized in Fig. 1. 


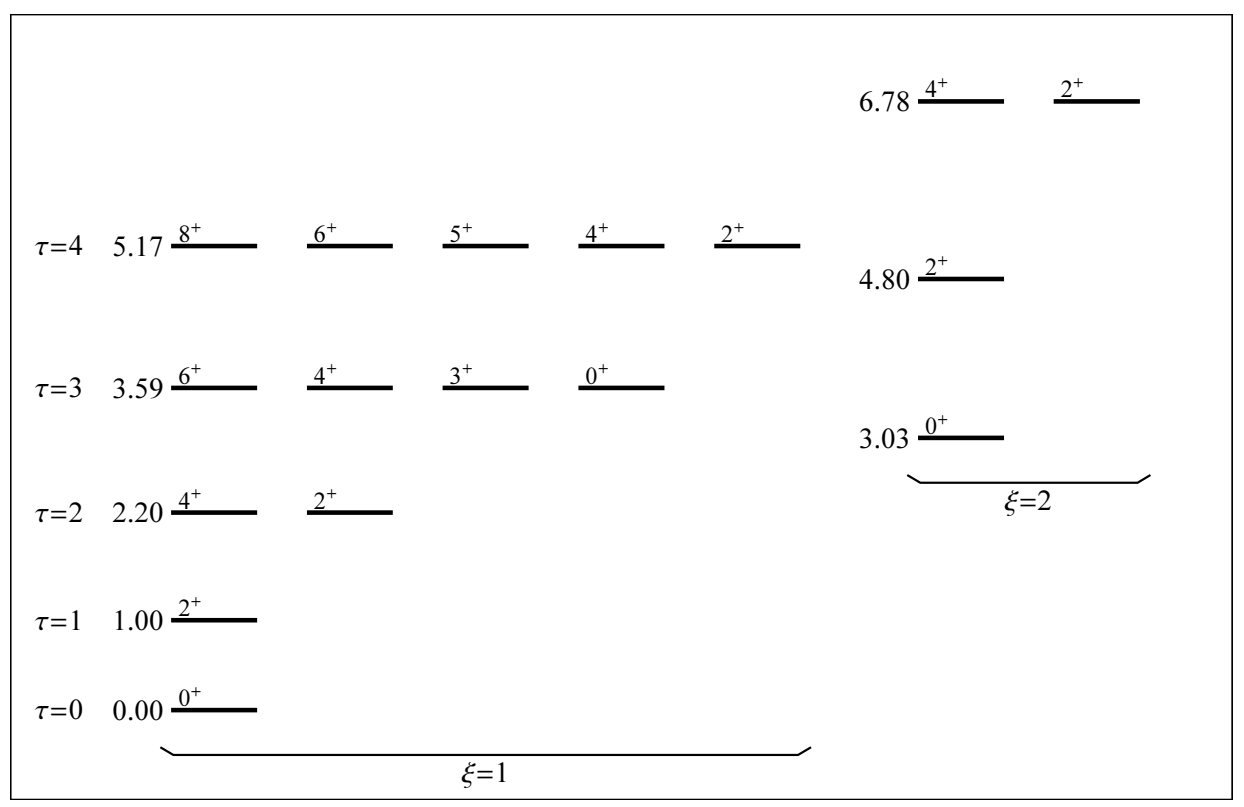

Fig. 1. Level scheme for the $\mathrm{E}(5)$ model, showing the excitation energies of the lowest $\tau$ multiplets of the first two $\xi$ families. All energies are normalized to $E\left(2_{1,1}^{+}\right)$.

\subsection{Classification of states}

Exactly solvable problems are usually characterized by the presence of dynamical symmetries (e.g., Refs. [2,44]). Therefore, an interesting question is the extent to which the square well problem is amenable to group theoretical treatment.

We begin by recognizing that the Hamiltonian (2.1) can be written, within the well boundary $\left(\beta \leq \beta_{W}\right)$, as the five-dimensional free Hamiltonian

$$
H=\frac{\hbar^{2}}{2 B} \tilde{\pi} \cdot \tilde{\pi}
$$

The five-dimensional rotation algebra $\mathrm{SO}(5)$, already discussed, has as its generators the "five-dimensional angular momentum operators" $\hat{\Lambda}_{i j}$, ten in number $[6,45]$. Here it is most useful to reexpress these in Racah (or spherical tensor) form, as

$$
\hat{\Lambda}_{\mu}^{(\lambda)} \equiv i \sqrt{2}(\alpha \times \tilde{\pi})_{\mu}^{(\lambda)},
$$

where $\lambda=1$ and 3. The Casimir operator of $\mathrm{SO}(5)$ is $C_{2}[\mathrm{SO}(5)]=2 \hat{\Lambda} \circ \hat{\Lambda}=$ $2\left[\hat{\Lambda}^{(1)} \cdot \hat{\Lambda}^{(1)}+\hat{\Lambda}^{(3)} \cdot \hat{\Lambda}^{(3)}\right]$, with eigenvalue $2 \tau(\tau+3)$, where an open dot indicates the $\mathrm{SO}(5)$ scalar product. The ordinary three-dimensional angular momentum operators $\hat{L}_{\mu}$, which generate $\mathrm{SO}(3)$, are

$$
\hat{L}_{\mu} \equiv i \sqrt{10}(\alpha \times \tilde{\pi})_{\mu}^{(1)}=\sqrt{5} \hat{\Lambda}_{\mu}^{(1)} .
$$


The $\mathrm{SO}(3)$ Casimir operator is simply $C_{2}[\mathrm{SO}(3)]=2 \hat{L} \cdot \hat{L}$, with eigenvalue $2 L(L+1)$. The algebras $\mathrm{SO}(5)$ and $\mathrm{SO}(3)$ can furthermore be embedded in the Euclidean algebra E(5). This is the (non-semisimple) algebra which generates translations and rotations in five dimensions. The generators, in spherical tensor form, are the five linear momentum components $\tilde{\pi}_{\mu}$ taken together with the ten angular momentum components $\hat{\Lambda}_{\mu}^{(\lambda)}$. The free Hamiltonian (2.16) is, naturally, invariant under both translations and rotations in five-dimensional space and is thus recognized as the Casimir operator

$$
C_{2}[\mathrm{E}(5)]=\tilde{\pi} \cdot \tilde{\pi}
$$

A dynamical symmetry is a situation in which the Hamiltonian is constructed from the Casimir operators of the algebras in a subalgebra chain [1, $2,44]$. The eigenvalues of the Casimir operators provide good quantum numbers for the eigenstates. The algebras just described form the subalgebra chain $\mathrm{E}(5) \supset \mathrm{SO}(5) \supset \mathrm{SO}(3) \supset \mathrm{SO}(2)$. The free Hamiltonian (2.16) describes a dynamical symmetry situation for this algebra chain, as $H=C_{2}[\mathrm{E}(5)]$. The square well Hamiltonian (2.1) therefore also describes a dynamical symmetry, provided that all operators are restricted to the bounded domain defined by $\beta \leq \beta_{W}$. Note that Casimir operators of the subalgebras $\mathrm{SO}(5)$ and $\mathrm{SO}(3)$ can also be included in the Hamiltonian, as discussed in Sec. 2.5. The Bessel functions form bases for the representation of the Euclidean algebras, as discussed in Ref. [46]. In the present five-dimensional case, the wave functions $\Phi(\beta, \gamma, \vartheta) \propto \beta^{-3 / 2} J_{\tau+3 / 2}\left(\varepsilon^{1 / 2} \beta\right) \Psi_{\tau \tilde{\nu}_{\triangle} L M_{L}}(\gamma, \vartheta)$ are characterized by the $\mathrm{E}(5)$ quantum number $\left\langle C_{2}[\mathrm{E}(5)]\right\rangle=\varepsilon$. For the free problem $\varepsilon$ may vary continuously $(\varepsilon \geq 0)$. For the square well problem, the node condition at the boundary instead restricts $\varepsilon$ to discrete values (2.13). To summarize, the free or square well Hamiltonians are both dynamical symmetry situations, characterized by the subalgebra chain

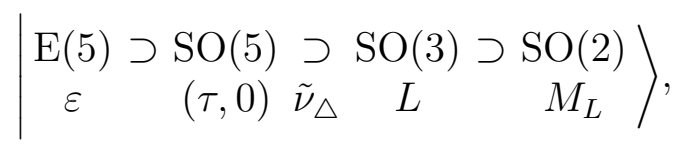

and the eigenstates are members of representations with the quantum numbers indicated.

However, some important practical differences should be noted between the present E(5) dynamical symmetry and others encountered in either geometric [47] or algebraic [2,3] problems. Many of the calculational simplicities usually associated with a dynamical symmetry arise from the presence of a spectrum generating algebra, which provides ladder operators allowing the various states in the model to be constructed from each other. For the generalized oscillator potential (2.9) and generalized Coulomb potential (2.10), in any number of dimensions, the spectrum generating algebras are known. 
Namely, in the present five-dimensional case, the algebras are $\operatorname{Sp}(10, R)$ or $\mathrm{U}(5,1)$ for the oscillator potential $[47$, p. 290] and $\mathrm{SO}(6,2)$ for the Coulomb potential [2, p. 184]. For the square well problem, the boundary condition makes construction of the ladder operators considerably more involved. The spectrum generating algebra for the square well has been explicitly constructed only in one dimension, where it is $\mathrm{SU}(1,1) \approx \mathrm{SO}(2,1)$, in a quantum number dependent realization [48-50]. An explicit construction in more than one dimension remains to be done.

\subsection{Electromagnetic transition strengths}

\subsubsection{General properties}

Electromagnetic transition strengths play an essential role in establishing the extent to which the present description applies to nuclei near the phase transition. The various terms contributing to the electromagnetic transition operators transform as $\mathrm{SO}(5)$ tensors, and the $\mathrm{E}(5)$ eigenstates posess $\mathrm{SO}(5)$ tensor character as well. Consequently, the pattern of transitions between levels is determined by $\mathrm{SO}(5)$ selection rules. Typically, either the transition between two levels is forbidden for the leading order term in the transition operator but allowed for the next higher order term or vice versa. It is thus especially important in the present problem to consider terms beyond leading order in the transition operators.

\subsubsection{E2 transitions}

Let us first consider electric quadrupole (E2) transitions. The general E2 operator may be expanded in terms of the coordinates $\alpha(2.2)$ as

$$
T^{(E 2)}=t_{21} \alpha+t_{22}(\alpha \times \alpha)^{(2)}+\cdots .
$$

The operator with first and second order terms is analogous to the $E 2$ operator commonly used in the IBM. Microscopic derivations within the context of the IBM [51] suggest that the contribution of the second order term is of the same order of magnitude as that of the first order term, as do phenomenological studies of actual nuclei [52]. Transition strengths are related to the reduced matrix elements by

$$
B\left(E 2 ; \xi \tau L \rightarrow \xi^{\prime} \tau^{\prime} L^{\prime}\right)=\frac{1}{2 L+1}\left\langle\xi^{\prime} \tau^{\prime} L^{\prime}\left\|T^{(E 2)}\right\| \xi \tau L\right\rangle^{2}
$$


and quadrupole moments by

$$
e Q(\xi \tau L)=\sqrt{\frac{16 \pi}{5}}\left[\frac{L(2 L-1)}{(2 L+1)(L+1)(2 L+3)}\right]^{1 / 2}\left\langle\xi \tau L\left\|T^{(E 2)}\right\| \xi \tau L\right\rangle .
$$

The explicit expression for the leading order term $\alpha$ in terms of Bohr variables is given in (2.2), while the second order term $(\alpha \times \alpha)^{(2)}$ is given by

$$
(\alpha \times \alpha)_{\mu}^{(2)}=\sqrt{\frac{2}{7}} \beta^{2}\left[-\cos 2 \gamma \mathscr{D}_{\mu 0}^{(2)}(\vartheta)+\frac{1}{\sqrt{2}} \sin 2 \gamma\left[\mathscr{D}_{\mu 2}^{(2)}(\vartheta)+\mathscr{D}_{\mu-2}^{(2)}(\vartheta)\right]\right] .
$$

Each of these operators, $\alpha$ or $(\alpha \times \alpha)^{(2)}$, factors into a radial part $\left(\beta\right.$ or $\left.\beta^{2}\right)$ and an angular part (involving $\gamma$ and $\vartheta$ ). Its reduced matrix element between $\mathrm{E}(5)$ eigenstates is thus the product of a radial matrix element and an angular reduced matrix element. The radial matrix element

$$
\int_{0}^{\beta_{W}} \beta^{4} d \beta f_{\xi^{\prime} \tau^{\prime}}(\beta) \beta^{m} f_{\xi \tau}(\beta)
$$

is evaluated by numerical integration, using the wave functions from Eq. (2.14). [The radial wave functions obtained for different values of the well width $\beta_{W}$ are related by a dilation in the radial coordinate $\beta$, as seen from (2.14), and consequently radial matrix elements scale as $\beta_{W}^{m}$.] Once the angular wave functions $\Psi_{\tau \tilde{\nu} \Delta L M_{L}}(\gamma, \vartheta)$ are explicitly constructed as in Eq. (2.7), evaluation of the angular matrix elements is straightforward. For a general spherical tensor operator $f_{\mu}^{(\lambda)}(\gamma, \vartheta)=\sum_{\substack{\kappa=0 \\ \text { even }}}^{\lambda} f_{\kappa}^{\lambda}(\gamma) \phi_{\mu \kappa}^{\lambda}(\vartheta)$, the reduced matrix element is [53]

$$
\begin{aligned}
& \left\langle\Psi_{\tau^{\prime} \tilde{\nu}_{\triangle}^{\prime} L^{\prime}}\left\|f^{(\lambda)}(\gamma, \vartheta)\right\| \Psi_{\tau \tilde{\nu}_{\triangle} L}\right\rangle \\
& =\frac{1}{4 \pi}[(2 L+1)(2 \lambda+1)]^{1 / 2} \sum_{\substack{K^{\prime}, \kappa, K \\
\text { even }}}\left[\frac{1+\delta_{K^{\prime}}}{\left(1+\delta_{K}\right)\left(1+\delta_{\kappa}\right)}\right]^{1 / 2} \\
& \times\left[\left(L K \lambda \kappa \mid L^{\prime} K^{\prime}\right)+\left\{\begin{array}{ll}
(-)^{\lambda}\left(L K \lambda \bar{\kappa} \mid L^{\prime} K^{\prime}\right) & K \geq \kappa \\
(-)^{L}\left(L \bar{K} \lambda \kappa \mid L^{\prime} K^{\prime}\right) & K \leq \kappa
\end{array}\right\}\right] \\
& \times\left[\int|\sin 3 \gamma| d \gamma F_{\tau^{\prime} \tilde{\nu}_{\triangle}^{\prime} L^{\prime} K^{\prime}}^{*}(\gamma) f_{\kappa}^{\lambda}(\gamma) F_{\tau \tilde{\nu} \triangle L K}(\gamma)\right] \text {, }
\end{aligned}
$$

where we follow Racah's normalization convention [54] for the Wigner-Eckart theorem. For the transition operators considered here, the integrals only involve polynomials in trigonometric functions and may therefore be evaluated exactly. The resulting angular matrix elements are tabulated in Appendix C. Evaluation of the angular matrix elements can also be carried out using algebraic methods [42].

The operator $\alpha$ is proportional to the angular wave function $\Psi_{102}(\gamma, \vartheta)$. It thus transforms as a $(1,0)$ tensor under $\mathrm{SO}(5)$ and can only connect states 


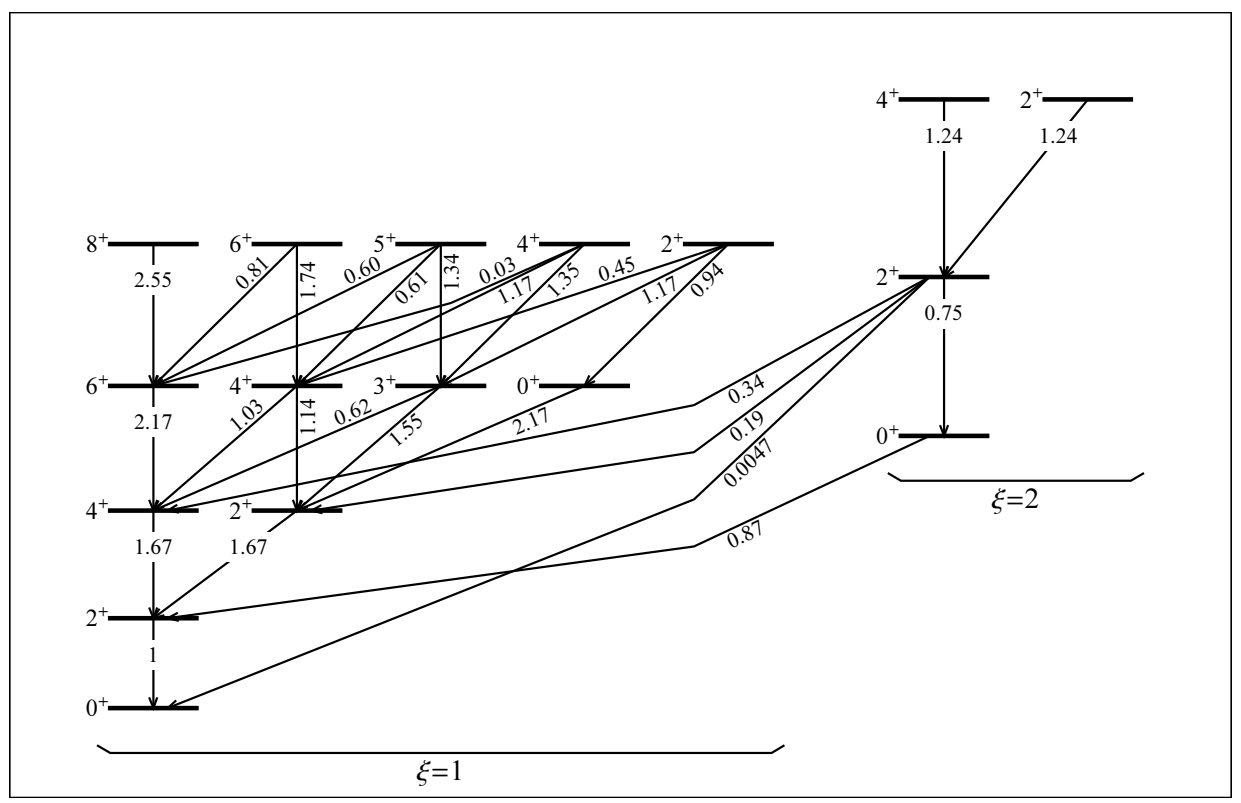

Fig. 2. $B(E 2)$ strengths induced by the operator $t_{21} \alpha$, for the $E(5)$ model. Transitions obey the selection rule $\Delta \tau=1$. All transition strengths are normalized relative to $B\left(E 2 ; 2_{1}^{+} \rightarrow 0_{1}^{+}\right)$. For absolute values, strengths should be multiplied by $(0.07453) t_{21}^{2} \beta_{W}^{2}$.

related by $\Delta \tau= \pm 1$. The transition strengths induced by $\alpha$ are shown in Fig. 2 . All quadrupole moments vanish if only this leading-order contribution to the E2 operator is considered.

The operator $(\alpha \times \alpha)^{(2)}$ is instead proportional to the angular wave function $\Psi_{202}(\gamma, \vartheta)$. It thus transforms as a $(2,0)$ tensor under $\mathrm{SO}(5)$ and connects states related by $\Delta \tau=0$ or \pm 2 . Consequently, it can yield nonzero quadrupole moments. The transition strengths induced by $(\alpha \times \alpha)^{(2)}$ are shown in Fig. 3, and the quadrupole moments are shown in Fig. 4. Note that the second order E2 operator in the E(5) model was also considered in Ref. [22], but a modified radial dependence $\propto 1 /\left(1+\beta^{2}\right)$ was used for the transition operator in that work, so slightly different results are obtained.

The operators $\alpha$ and $(\alpha \times \alpha)^{(2)}$ contribute to entirely distinct sets of transitions due to the selection rules in the $\mathrm{E}(5)$ problem. Consequently, interference between them never occurs. The parameter $t_{21}$ determines the overall normalization of all $\Delta \tau= \pm 1$ transition strengths. Predictions for all strength ratios of $\Delta \tau= \pm 1$ transitions are parameter independent within the $\mathrm{E}(5)$ model. Similarly, $t_{22}$ determines the overall normalization of all $\Delta \tau=0$ or \pm 2 transition strengths and the quadrupole moments. The predictions are discussed further in Sec. 2.4. 


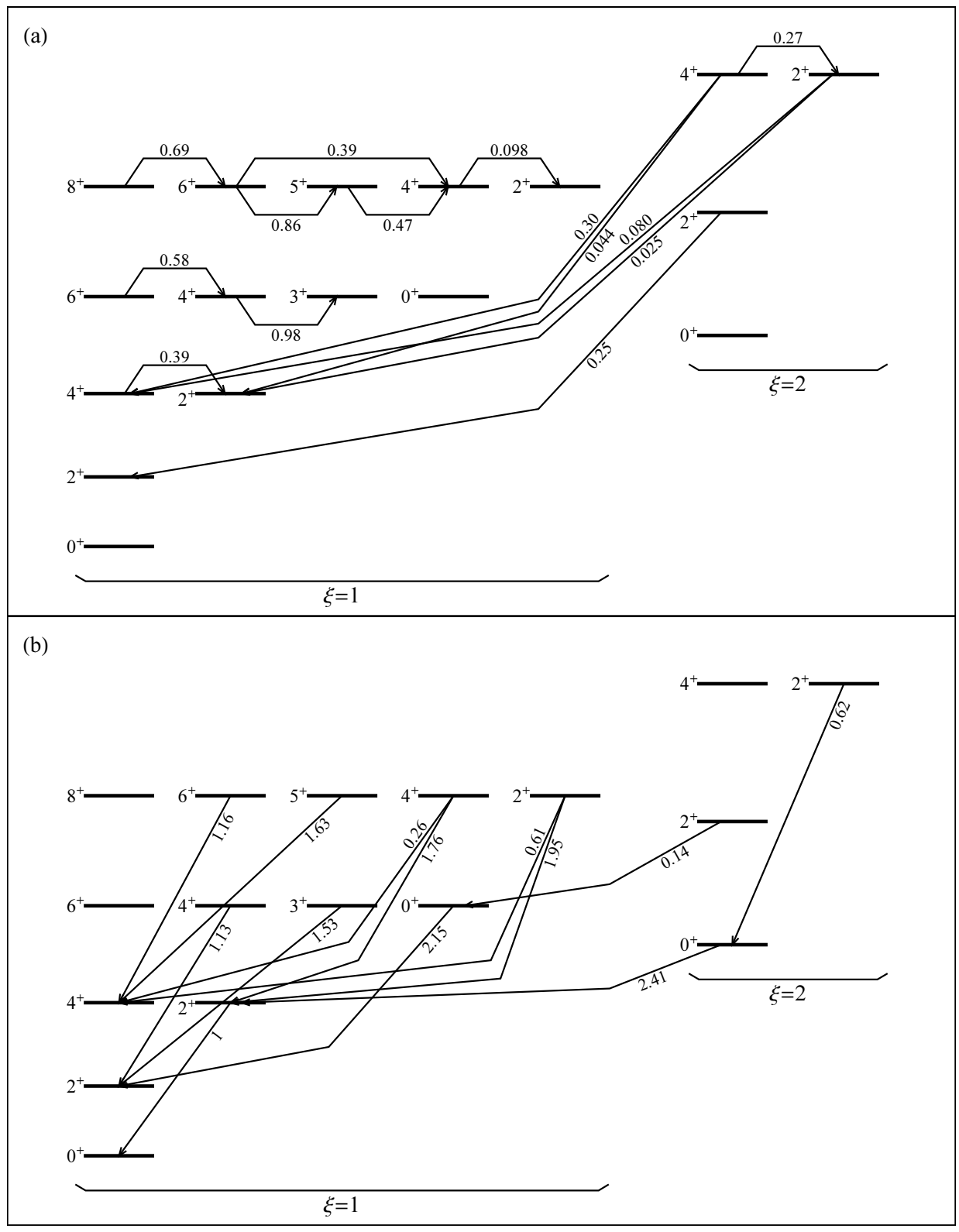

Fig. 3. $B(E 2)$ strengths induced by the operator $t_{22}(\alpha \times \alpha)^{(2)}$, for the $\mathrm{E}(5)$ model: (a) transitions with $\Delta \tau=0$ and (b) transitions with $\Delta \tau= \pm 2$. All transition strengths are normalized relative to $B\left(E 2 ; 2_{2}^{+} \rightarrow 0_{1}^{+}\right)$. For absolute values, strengths should be multiplied by $(0.009754) t_{22}^{2} \beta_{W}^{4}$.

\subsubsection{M1 transitions}

Magnetic dipole transitions arise from differences between the proton and neutron contributions to collective motion. They are therefore most naturally treated in a model which distinguishes proton and neutron degrees of freedom $[51,55]$. However, within the Bohr picture, an effective $M 1$ operator may 


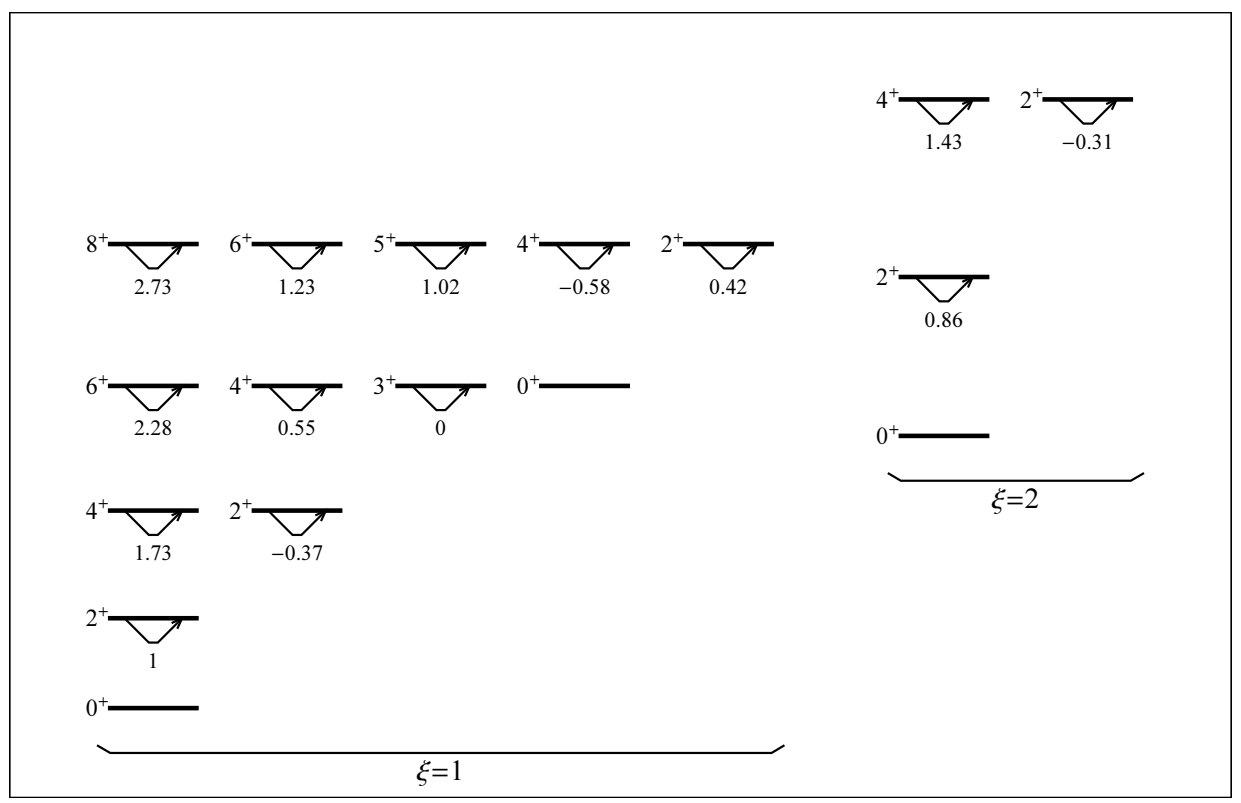

Fig. 4. Quadrupole moments induced by the operator $t_{22}(\alpha \times \alpha)^{(2)}$, for the $\mathrm{E}(5)$ model. All moments are normalized relative to $Q\left(2_{1}^{+}\right)$. For absolute values, moments should be multiplied by $(0.2124) t_{22} \beta_{W}^{2}$.

be expressed in terms of $\alpha$ and $\tilde{\pi}$ as $[6,56]$

$$
\begin{aligned}
T^{(M 1)} & =t_{10}\left[i(\alpha \times \tilde{\pi})^{(1)}\right]+t_{11}\left[\alpha \times i(\alpha \times \tilde{\pi})^{(1)}\right]^{(1)}+\cdots \\
& =t_{10} \frac{1}{\sqrt{10}} \hat{L}+t_{11} \frac{1}{\sqrt{10}}(\alpha \times \hat{L})^{(1)}+\cdots .
\end{aligned}
$$

This is closely analogous to the effective $M 1$ operator used in the IBM [3]. Transition strengths are given by

$$
B\left(M 1 ; \xi \tau L \rightarrow \xi^{\prime} \tau^{\prime} L^{\prime}\right)=\frac{1}{2 L+1}\left\langle\xi^{\prime} \tau^{\prime} L^{\prime}\left\|T^{(M 1)}\right\| \xi \tau L\right\rangle^{2},
$$

and magnetic dipole moments by

$$
\mu(\xi \tau L)=\sqrt{\frac{4 \pi}{3}}\left[\frac{L}{(2 L+1)(L+1)}\right]^{1 / 2}\left\langle\xi \tau L\left\|T^{(M 1)}\right\| \xi \tau L\right\rangle .
$$

Since the leading order term in $T^{(M 1)}$ is simply proportional to the collective angular momentum operator $\hat{L}$, it is diagonal in the eigenfunctions and cannot induce transitions. It yields magnetic moments which depend only upon $L$,

$$
\mu(\xi \tau L)=\sqrt{\frac{4 \pi}{3}} t_{10} L=g_{B} L,
$$

where $g_{B} \equiv(4 \pi / 3)^{1 / 2} t_{10}$, as usual in the collective picture. However, the higher order $M 1$ operator $[\alpha \times \hat{L}]^{(1)}$ does yield nonvanishing transitions. The matrix 


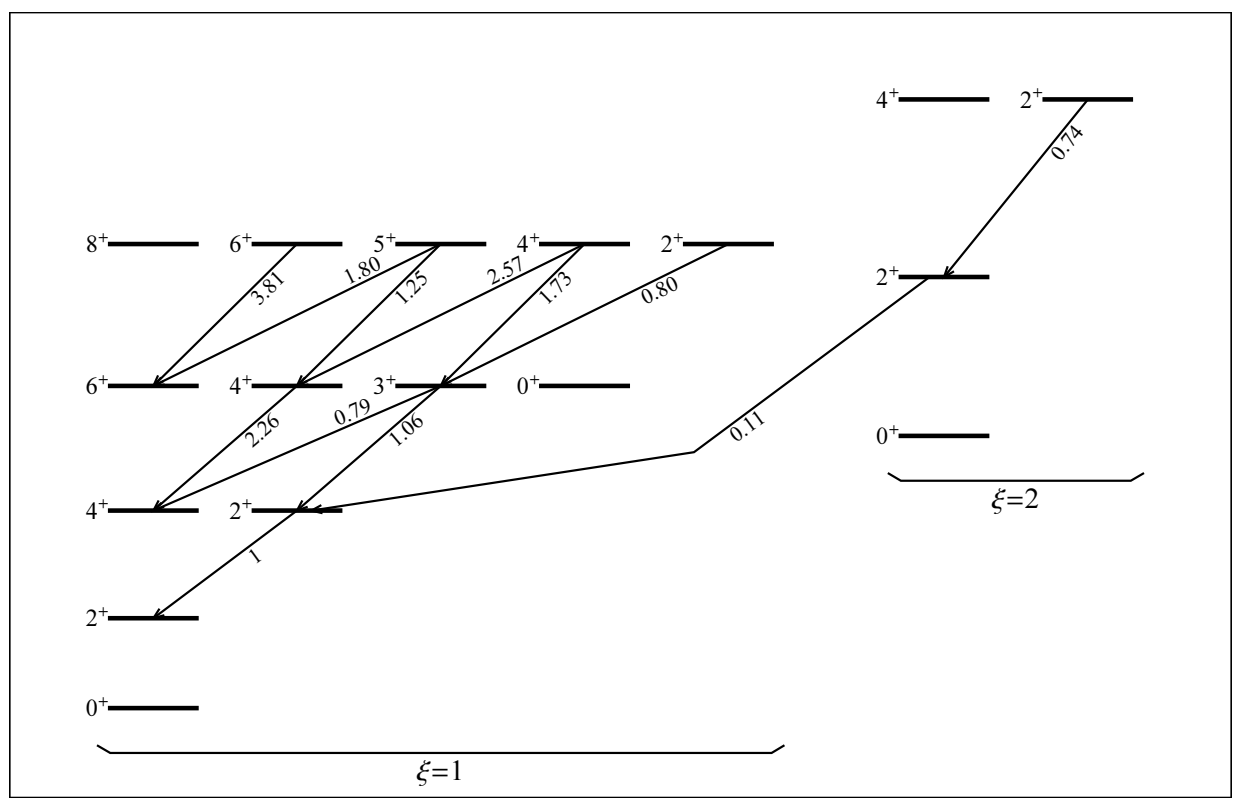

Fig. 5. $B(M 1)$ strengths induced by the operator $t_{11}\left[\alpha \times(\alpha \times \pi)^{(1)}\right]^{(1)}$, for the $\mathrm{E}(5)$ model. Transitions obey the selection rule $\Delta \tau=1$. All transition strengths are normalized relative to $B\left(M 1 ; 2_{2}^{+} \rightarrow 2_{1}^{+}\right)$. For absolute values, strengths should be multiplied by $(0.02620) t_{11}^{2} \beta_{W}^{2}$.

elements follow from those already calculated above for $\alpha$ according to the Racah reduction formula [54], giving

$$
\begin{aligned}
& \left\langle\xi^{\prime} \tau^{\prime} L^{\prime}\left\|(\alpha \times \hat{L})^{(1)}\right\| \xi \tau L\right\rangle \\
& =-\frac{1}{\sqrt{40}}\left[\left(L-L^{\prime}+2\right)\left(-L+L^{\prime}+2\right)\left(L+L^{\prime}-1\right)\left(L+L^{\prime}+3\right)\right]^{1 / 2} \\
& \quad \times\left\langle\xi^{\prime} \tau^{\prime} L^{\prime}\|\alpha\| \xi \tau L\right\rangle .
\end{aligned}
$$

The operator follows the same $\Delta \tau= \pm 1$ selection rule as $\alpha$ (but with the more restrictive angular momentum selection rule appropriate to a dipole operator). The resulting transition strengths are shown in Fig. 5.

\subsubsection{E0 transitions}

The collective electric monopole operator is of the form

$$
\begin{aligned}
T^{(E 0)} & =t_{00}(\alpha \times \alpha)^{(0)}+\cdots \\
& =t_{00} \frac{1}{\sqrt{5}} \beta^{2}+\cdots .
\end{aligned}
$$


Table 2

Selected $\rho^{2}(E 0)$ strengths for the E(5) model. All $\rho^{2}(E 0)$ strengths are normalized relative to $\rho\left(E 0 ; 0_{\xi=2}^{+} \rightarrow 0_{1}^{+}\right)$. For absolute values, strengths should be multiplied by $(0.006343) t_{02}^{2} \beta_{W}^{4} /\left(e R^{2}\right)^{2}$.

\begin{tabular}{rrrrrrr}
\hline$\xi$ & $\tau$ & $J$ & $\xi^{\prime}$ & $\tau^{\prime}$ & $J^{\prime}$ & $\rho^{2}\left(E 0 ; J \rightarrow J^{\prime}\right)$ \\
\hline 2 & 0 & 0 & 1 & 0 & 0 & 1.0000 \\
2 & 1 & 2 & 1 & 1 & 2 & 0.9234 \\
2 & 2 & 2 & 1 & 2 & 2 & 0.8426 \\
2 & 2 & 4 & 1 & 2 & 4 & 0.8426 \\
\hline
\end{tabular}

Transition strengths are usually quoted as squared $\rho(E 0)$ values, defined by $[57]$

$$
\begin{aligned}
\left(e R^{2}\right) \rho\left(E 0 ; \xi \tau L \rightarrow \xi^{\prime} \tau^{\prime} L\right) & =\frac{1}{\sqrt{2 L+1}}\left\langle\xi^{\prime} \tau^{\prime} L\left\|T^{(E 0)}\right\| \xi \tau L\right\rangle \\
& =\left\langle\xi^{\prime} \tau^{\prime} L L\left|T^{(E 0)}\right| \xi \tau L L\right\rangle
\end{aligned}
$$

where $R$ is the nuclear radius. The leading order operator $\beta^{2}$ consists only of a radial factor, so evaluation of the matrix elements involves only the radial integral (2.25). Transitions occur only between states with identical angular quantum numbers. Selected transition strengths are listed in Table 2.

\subsection{Comparison with experimental data}

Since $\mathrm{SO}(5)$ symmetry is present throughout the transition between spherical and deformed $\gamma$-soft structure, comparison of the $\mathrm{E}(5)$ predictions with experimental data is not simple. Many of the gross spectroscopic features, including the level multiplet structure and electromagnetic branching ratios, follow from the $\mathrm{SO}(5)$ symmetry and therefore persist throughout the transition. In this section, we therefore consider the distinguishing observables which do vary along the $\mathrm{U}(5)-\mathrm{SO}(6)$ transition.

The most fundamental energy ratios from a spectroscopic viewpoint are

$$
E\left(4_{1,2}^{+}\right) / E\left(2_{1,1}^{+}\right)=E\left(2_{1,2}^{+}\right) / E\left(2_{1,1}^{+}\right)=2.20
$$

and

$$
E\left(0_{2,0}^{+}\right) / E\left(2_{1,1}^{+}\right)=3.03
$$

These place the yrast $E\left(4^{+}\right) / E\left(2^{+}\right)$ratio intermediate between the $\mathrm{U}(5)$ value of 2 and the $\mathrm{SO}(6)$ value of 2.5 . They also provide an estimate for the excitation energy of the first radial $(\xi)$ excitation. The $B(E 2)$ strength ratios involving 

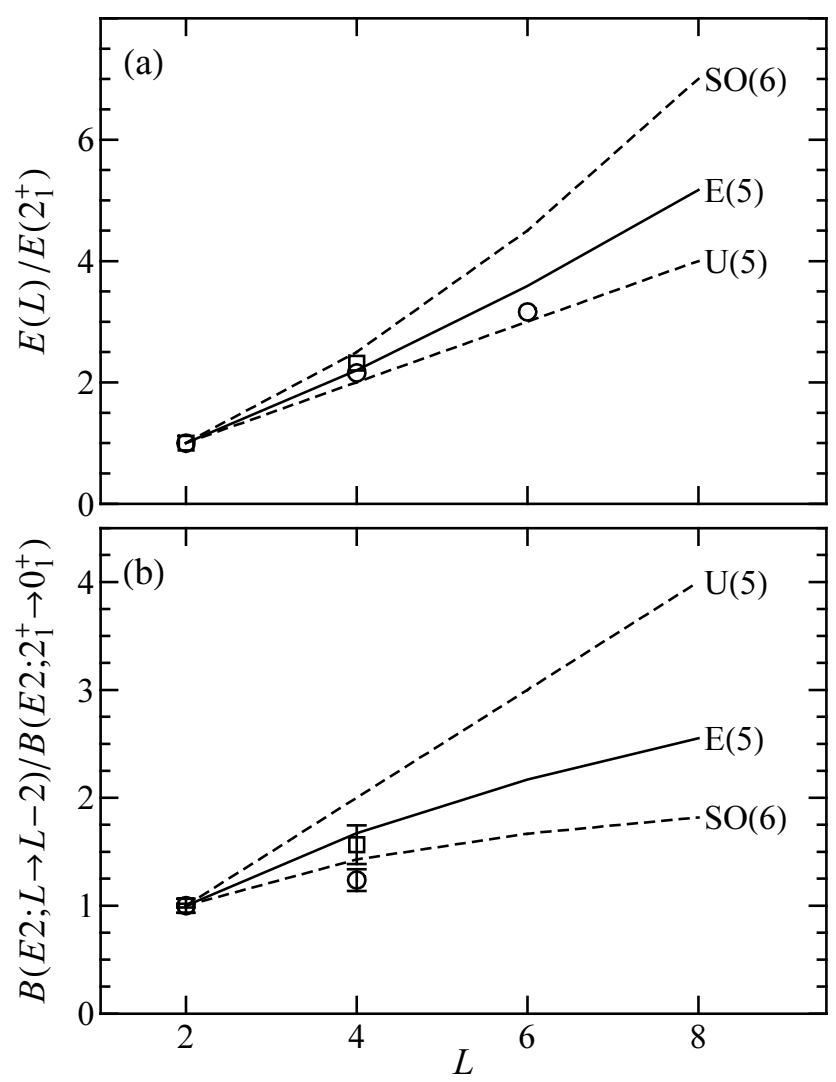

Fig. 6. Yrast (a) energies and (b) $B(E 2)$ strengths as functions of angular momentum in the $\mathrm{E}(5)$ model. The experimental data for ${ }^{132} \mathrm{Xe}(\mathrm{O})$ and ${ }^{134} \mathrm{Ba}(\square)[58,59]$ are indicated for comparison, as are the predictions for the $\mathrm{U}(5)$ and $\mathrm{SO}(6)$ dynamical symmetries in the large boson number limit (dashed curves). Energies are normalized to $E\left(2_{1}^{+}\right)$and $B(E 2)$ strengths to $B\left(E 2 ; 2_{1}^{+} \rightarrow 0_{1}^{+}\right)$.

these same levels are

$$
\frac{B\left(E 2 ; 4_{1,2}^{+} \rightarrow 2_{1,1}^{+}\right)}{B\left(E 2 ; 2_{1,1}^{+} \rightarrow 0_{1,0}^{+}\right)}=\frac{B\left(E 2 ; 2_{1,2}^{+} \rightarrow 2_{1,1}^{+}\right)}{B\left(E 2 ; 2_{1,1}^{+} \rightarrow 0_{1,0}^{+}\right)}=1.68
$$

and

$$
\frac{B\left(E 2 ; 0_{2,0}^{+} \rightarrow 2_{1,1}^{+}\right)}{B\left(E 2 ; 2_{1,1}^{+} \rightarrow 0_{1,0}^{+}\right)}=0.68
$$

The properties of the yrast states as a function of angular momentum are also among the most relevant observables. They vary continuously along the $\mathrm{U}(5)-\mathrm{SO}(6)$ transition, and they are among the most feasible observables to measure. The yrast excitation energy ratios $E\left(L^{+}\right) / E\left(2_{1,1}^{+}\right)$are shown in Fig. 6(a). The yrast $B(E 2)$ ratios $B\left[E 2 ; L^{+} \rightarrow(L-2)^{+}\right] / B\left(E 2 ; 2_{1,1}^{+} \rightarrow 0_{1,0}^{+}\right)$ are shown in Fig. 6(b). Casten and Zamfir [21] have suggested that ${ }^{134} \mathrm{Ba}$ is 
well described by the E(5) predictions. The measured yrast observables for ${ }^{134} \mathrm{Ba}$ and ${ }^{132} \mathrm{Xe}$, as well as the predictions for the $\mathrm{U}(5)$ and $\mathrm{SO}(6)$ limits of the transition, are shown for comparison in Fig. 6.

A distinguishing feature of the evolution along the transition from spherical to deformed structure is the increase in energy of the radial excitation, specifically the $0^{+}$head of the excited family of levels. For the U(5) limit, $E\left(0^{+}\right) / E\left(2_{1}^{+}\right)=2$. The $\mathrm{E}(5)$ prediction is $E\left(0^{+}\right) / E\left(2_{1}^{+}\right)=3.03$, as noted above. The prediction for true "rigidly" $\beta$-deformed, $\gamma$-soft structure would be $E\left(0^{+}\right) / E\left(2_{1}^{+}\right) \rightarrow \infty$. For more realistic situations, the result is dependent upon the stiffness of the potential [14] in the geometric picture or upon the $\mathrm{SO}(6)$ Hamiltonian coefficients (and boson number) in the algebraic picture [42]. There is thus no firm limiting $\mathrm{SO}(6)$ value for this ratio from theory alone. Rather, the limit must be considered empirically, suggesting $E\left(0^{+}\right) / E\left(2_{1}^{+}\right) \approx 4$.

An important consideration in experimental determination of the properties of the radial excitation is the need to distinguish between the $0_{2,0}^{+}$state (radial or $\xi$ excitation) and the $0_{1,3}^{+}$state (member of the $\tau=3$ multiplet of the ground state family) which may lie nearby in energy [25]. In many of the nuclei of experimental interest, the situation is further complicated by the presence of intruder configurations [25].

Several nuclei, including various $\mathrm{Ru}, \mathrm{Pd}$, and $\mathrm{Ba}$ isotopes, have been considered as candidates for description by the E(5) model. Detailed comparisons between theory and experiment may be found in Refs. [21-23, 25, 26]. It has been suggested that E(5) structure may also be present in light nuclei [29].

\subsection{Generalizations of the $\mathrm{E}(5)$ description}

A simple solution such as that described so far can provide a qualitative description of structure near the critical point of the $\mathrm{U}(5)-\mathrm{SO}(6)$ transition, but it is naturally limited as a model for detailed description. Here we summarize generalizations of the $\mathrm{E}(5)$ description addressing some of the most significant physical issues.

Empirically, it is found that $\mathrm{SO}(5)$ level multiplets are often significantly split in energy, but in a fashion which depends monotonically upon the angular momentum. The splitting can be reproduced trivially by the introduction of a term proportional to $C_{2}[\mathrm{SO}(3)]$ in the Hamiltonian, which leaves the wave functions unchanged and perturbs the energies as $L(L+1)$. This is the usual dynamical symmetry approach, in which the Hamiltonian is simply written as a sum of Casimir operators of a chain of subalgebras (see Sec. 2.2), which for 


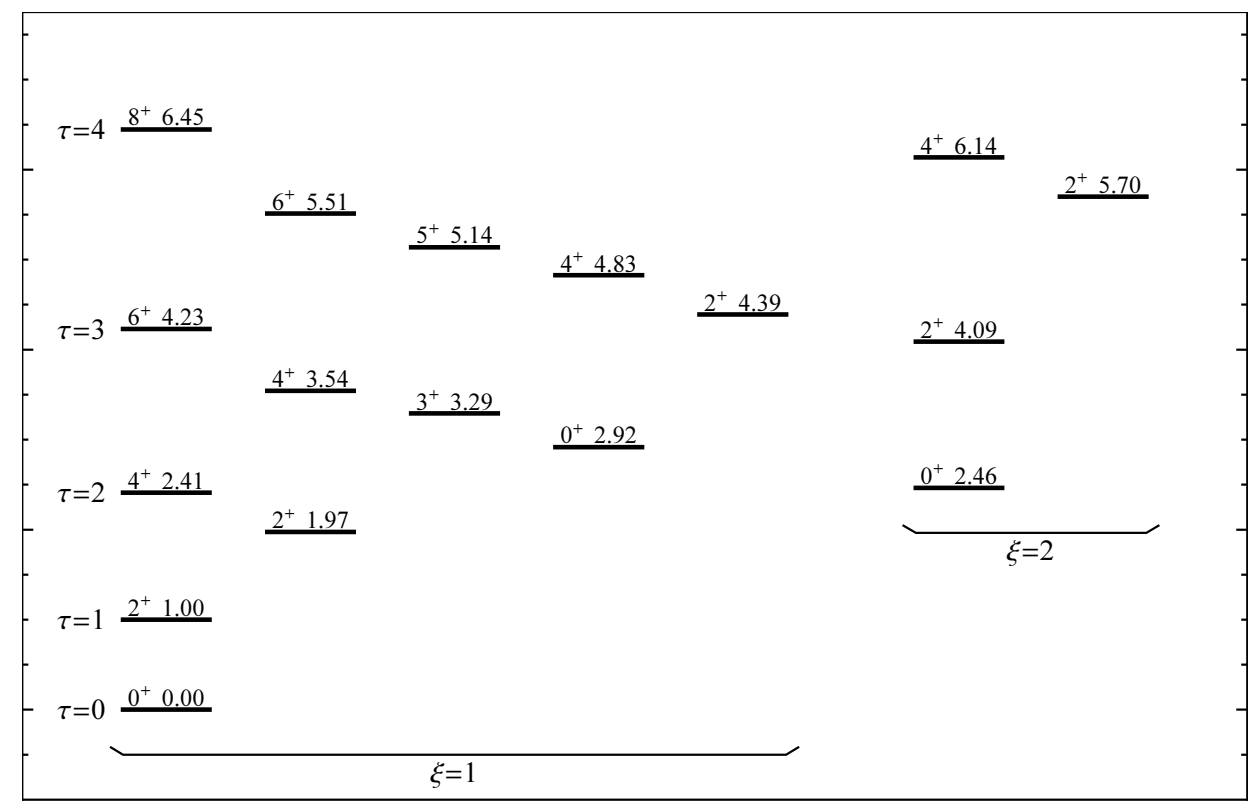

Fig. 7. Level scheme for the for the E(5) model with angular momentum degeneracy breaking term $k^{\prime} \hat{L} \cdot \hat{L}$, for $k^{\prime}=1 / 2$. All energies are normalized to $E\left(2_{1}^{+}\right)$.

chain (2.20) gives

$$
H=C_{2}[\mathrm{E}(5)]+c^{\prime \prime} C_{2}[\mathrm{SO}(5)]+c^{\prime} C_{2}[\mathrm{SO}(3)]
$$

or, explicitly in terms of the linear and angular momentum operators,

$$
H=\tilde{\pi} \cdot \tilde{\pi}+V(\beta)+k^{\prime \prime} \hat{\Lambda} \circ \hat{\Lambda}+k^{\prime} \hat{L} \cdot \hat{L} .
$$

An example level scheme is shown in Fig. 7. The evolution of energies with respect to the splitting parameter $k^{\prime}$ is shown in Fig. 8. Transition strengths are unaffected by the additional terms.

Much more general interactions can be introduced while still retaining an exactly solvable radial problem, and these are valuable to consider since they provide flexibility in the treatment of physical interactions. Any term proportional to to $1 / \beta^{2}$ can be absorbed into the separaton constant, and $\beta$ dependences of the form (2.9) or (2.10) can also be accomodated, so there is considerable freedom to include terms $\hat{\Lambda} \circ \hat{\Lambda} / \beta^{2}, \hat{L} \cdot \hat{L} / \beta^{2},(\hat{\Lambda} \circ \hat{\Lambda}) \beta^{2},(\hat{L} \cdot \hat{L}) \beta^{2}$, etc. For illustration, we consider

$$
H=\tilde{\pi} \cdot \tilde{\pi}+V(\beta)+k^{\prime} \frac{\hat{L} \cdot \hat{L}}{\beta^{2}} .
$$

In this case, $C_{2}[\mathrm{E}(5)]=\tilde{\pi} \cdot \tilde{\pi}$ no longer commutes with $H$, since $1 / \beta^{2}$ is not translationally invariant. Therefore, the eigenstates of $H$ are not eigenstates of $C_{2}[\mathrm{E}(5)]$, and the label $\varepsilon$ of the chain (2.20) no longer applies. Proper labeling 


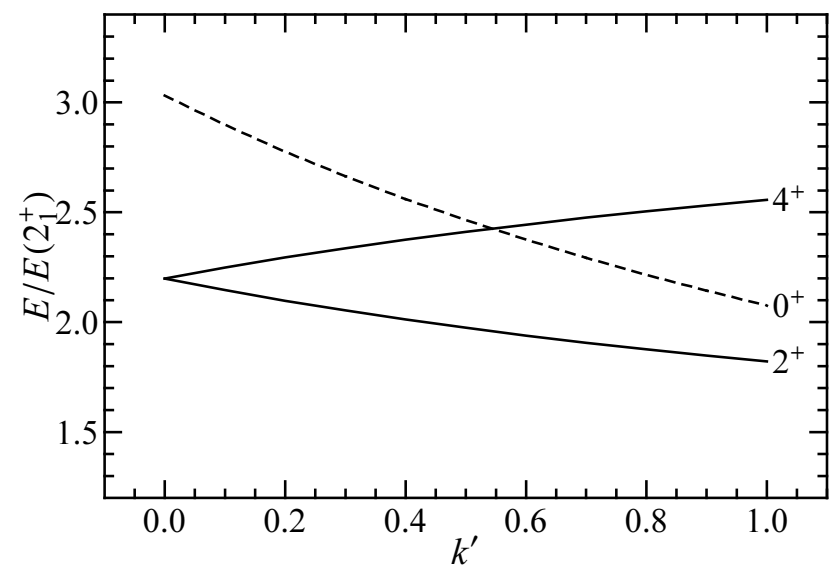

Fig. 8. Evolution of energies as functions of the angular momentum degeneracy breaking parameter $k^{\prime}$, for the E(5) model with degeneracy breaking term $k^{\prime} \hat{L} \cdot \hat{L}$. Energies are shown for the $\xi=1$ levels $4_{1}^{+}$and $2_{2}^{+}$(solid curves) and the $\xi=2$ family head $0_{\xi=2}^{+}$(dashed curve). Energies are normalized to $E\left(2_{1}^{+}\right)$.
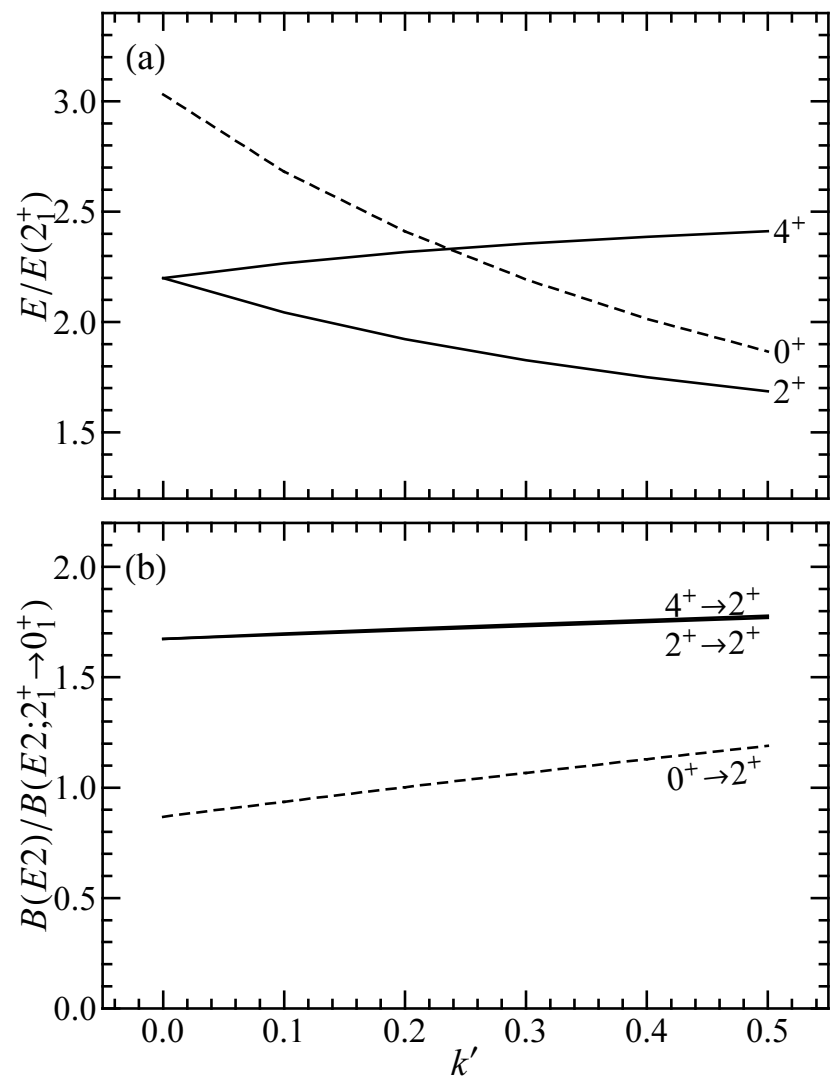

Fig. 9. Evolution of (a) energies and (b) $B(E 2)$ strengths for the $\mathrm{E}(5)$ model with $\beta$-dependent degeneracy breaking term $k^{\prime} \hat{L} \cdot \hat{L} / \beta^{2}$, for comparison with Fig. 8. Observables involving only the $\xi=1$ family of levels $\left[E\left(4_{1}^{+}\right), E\left(2_{2}^{+}\right), B\left(E 2 ; 4_{1}^{+} \rightarrow 2_{1}^{+}\right)\right.$, and $B\left(E 2 ; 2_{2}^{+} \rightarrow 2_{1}^{+}\right)$] are indicated by solid curves, while those involving the $\xi=2$ family head $\left[E\left(0_{\xi=2}^{+}\right)\right.$and $\left.B\left(E 2 ; 0_{\xi=2}^{+} \rightarrow 2_{1}^{+}\right)\right]$are indicated by dashed curves. Energies are normalized to $E\left(2_{1}^{+}\right)$and $B(E 2)$ strengths to $B\left(E 2 ; 2_{1}^{+} \rightarrow 0_{1}^{+}\right)$. 
requires the introduction of the $\mathrm{SO}(2,1)$ algebra for the radial problem [47]. In the separation of variables, the contribution of the additional angular momentum dependent term is absorbed into the separation constant $\Lambda$ in (2.6), so

$$
\Lambda=\tau(\tau+3)+k^{\prime} L(L+1)
$$

The eigenvalues and eigenfunctions are again given by Eqs. (2.13) and (2.14), but the order of the Bessel functions is now determined by the separation constant $\Lambda$ as given above, so $\nu(\tau, L)=\left[\tau(\tau+3)+k^{\prime} L(L+1)+9 / 4\right]^{1 / 2}$. The evolution of observables with respect to the degeneracy breaking parameter $k^{\prime}$ is shown in Fig. 9. The evolution of the energy splittings [Fig. 9(a)] differs little in its qualitative features from that obtained for the simpler degeneracy breaking term $k^{\prime} \hat{L} \cdot \hat{L}$ (Fig. 9), although a different numerical value of the parameter $k^{\prime}$ is needed to achieve the same magnitude effect in the two cases (note the different horizontal scales of Figs. 8 and 9). The electromagnetic observables are only weakly dependent upon the splitting interaction strength [Fig. 8(b)].

More fundamental limitations in the use of the E(5) Hamiltonian for realistic phenomenological analysis arise from the extreme nature of the infinite square well potential. The infinite depth per se is not a significant concern. Even for finite well depth, the tails of the radial wave functions outside the well wall are generally so suppressed that the actual depth does not matter in detail [60]. However, the infinite rigidity of the wall (infinite slope) introduces directly observable artifacts into the spectroscopic predictions. Most notably, the much greater energy spacing scale between levels in the $\xi=2$ family than in the $\xi=1$ family (Fig. 1) arises from the infinite slope of the wall and disappears for soft potentials. The detailed mechanism is discussed in Refs. [53, 61]. Comparisons of the $\mathrm{E}(5)$ results with those for potentials involving various polynomial dependences upon $\beta$ are given in Refs. [24, 27, 28, 62].

\section{Transitional Bose-Fermi symmetry}

\subsection{Hamiltonian and solution}

In the previous section, we have presented an analytic solution for the Bohr Hamiltonian which can be used in the description of even-even nuclei near the critical point of the spherical to $\gamma$-unstable transition. In this section, we construct an analytic solution for the Bohr Hamiltonian with coupling to an additional fermion. The study of dynamical symmetries of the coupled odd-mass system is much more involved than for the core alone [18]. The dynamical symmetries, called Bose-Fermi symmetries, can be constructed only in special circumstances. In the geometric formulation considered here, the 
fermion must be confined to one or more degenerate orbitals with suitable angular momenta. Namely, these orbitals must transform as a spinor representation of $\operatorname{Spin}(5)$ (e.g., $j=3 / 2[19]$ ) or as a tensor representation coupled to a spin in a pseudospin scheme (e.g., $j=3 / 2$ and $5 / 2$, obtained as $2 \otimes 1 / 2$ ). Furthermore, the core-fermion interaction must be of a special form, such that it can be written in terms of Casimir operators of the combined algebra of collective and single particle degrees of freedom. Analytic solutions can then be constructed using a method similar to that of the algebraic IBFM [18], but translated into a differential framework.

To treat the coupled system, we start from the generic Hamiltonian

$$
H=H_{B}+H_{F}+V_{B F} .
$$

The term $H_{B}$ is the Bohr Hamiltonian of Sec. 2, describing the core in isolation. We again restrict our attention to the case of a $\gamma$-independent potential, and to the square well in particular for transitional nuclei. The Hamiltonian $H_{F}$ is that of the single particle in a mean field, with eigenstates $\left|n \ell j m_{j}\right\rangle$ and eigenvalues which are simply the single particle energies $\varepsilon_{n \ell j m_{j}}$. For a set of degenerate orbitals, this $H_{F}$ is simply a constant and can henceforth be omitted. The interaction $V_{B F}$ introduces couplings between the collective coordinates and the single particle degrees of freedom.

Here we consider the case of a single particle with $j=3 / 2$, coupled to the nuclear quadrupole collective motion (rotations and vibrations of a liquid drop). The essential property which makes a solution possible is that a particle with $j=3 / 2$ and components $m_{j}=-3 / 2, \ldots,+3 / 2$ transforms as the representation $[1,0]$ of $\operatorname{Sp}(4)[63,64]$. However, $\operatorname{Sp}(4)$ is isomorphic to $\mathrm{SO}(5)$, providing a relationship between the transformation properties of the fermion and of the $\gamma$-soft core.

Specifically, a particle with $j=3 / 2$ is described by the subalgebra chain

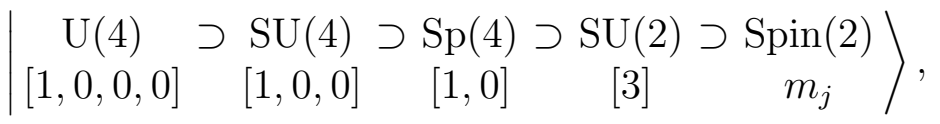

with the quantum numbers indicated. Making use of the isomorphisms $\mathrm{SU}(4) \approx \mathrm{SO}(6), \mathrm{Sp}(4) \approx \mathrm{SO}(5)$, and $\mathrm{SU}(2) \approx \mathrm{SO}(3)$, the particle can instead be characterized by its transformation properties under the angular momentum algebras $\mathrm{SO}(n)(n=6,5,3$, and 2$)$,

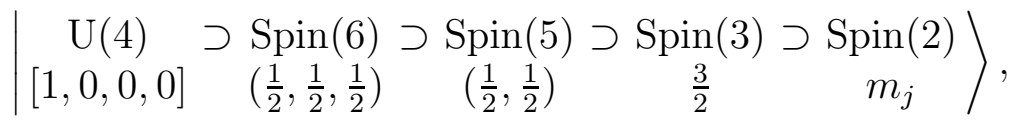

where the notation $\operatorname{Spin}(n)$ is used for $\mathrm{SO}(n)$ when spinor representations are involved [2]. To clarify the common five-dimensional angular momentum 
structure for the core and fermion, we explicitly note the Spin(5) generators. The five-dimensional angular momentum operators for the core $\left[\operatorname{Spin}_{B}(5) \equiv\right.$ $\mathrm{SO}(5)]$ are, from Sec. $2.2, \hat{\Lambda}_{\mu}^{(1)}=i \sqrt{2}(\alpha \times \tilde{\pi})_{\mu}^{(1)}$ and $\hat{\Lambda}_{\mu}^{(3)}=i \sqrt{2}(\alpha \times \tilde{\pi})_{\mu}^{(3)}$. The five-dimensional angular momentum operators for the fermion $\left[\operatorname{Spin}_{F}(5) \equiv\right.$ $\mathrm{Sp}(4)]$ are, from Appendix $\mathrm{A}, \hat{\Sigma}_{\mu}^{(1)}=-\left(a^{\dagger} \times \tilde{a}\right)_{\mu}^{(1)}$ and $\hat{\Sigma}_{\mu}^{(3)}=+\left(a^{\dagger} \times \tilde{a}\right)_{\mu}^{(3)}$, where $a^{\dagger}$ and $\tilde{a}$ are the fermion creation and annihilation operators. A total five-dimensional angular momentum algebra $\operatorname{Spin}_{B F}(5)$ is constructed from the sum generators $\hat{\Lambda}_{B F}=\hat{\Lambda}+\hat{\Sigma}$. The operators $\hat{\Lambda}, \hat{\Sigma}$, and $\hat{\Lambda}_{B F}$ all transform as $(1,1)$ tensors under $\operatorname{Spin}_{B F}(5)$.

The E(5|4) description, proposed in Ref. [20], is obtained by taking the core-fermion interaction to be of a special form, the five-dimensional analogue of a spin-orbit interaction. The interaction is given by the $\operatorname{Spin}(5)$ scalar product of the five-dimensional angular momenta,

$$
\hat{\Lambda} \circ \hat{\Sigma}=\hat{\Lambda}^{(1)} \cdot \hat{\Sigma}^{(1)}+\hat{\Lambda}^{(3)} \cdot \hat{\Sigma}^{(3)} .
$$

The Hamiltonian for the coupled system is then

$$
\begin{aligned}
H=-\frac{\hbar^{2}}{2 B}\left[\frac{1}{\beta^{4}} \frac{\partial}{\partial \beta} \beta^{4}\right. & \frac{\partial}{\partial \beta}+\frac{1}{\beta^{2}}\left(\frac{1}{\sin 3 \gamma} \frac{\partial}{\partial \gamma} \sin 3 \gamma \frac{\partial}{\partial \gamma}\right. \\
& \left.\left.-\frac{1}{4} \sum_{\kappa} \frac{\hat{L}_{\kappa}^{\prime 2}}{\sin ^{2}\left(\gamma-\frac{2}{3} \pi \kappa\right)}\right)\right]+V(\beta)+2 k g(\beta) \hat{\Lambda} \circ \hat{\Sigma}
\end{aligned}
$$

where we shall again set $\hbar^{2} /(2 B)=1$. The physical interpretation of the fivedimensional spin-orbit interaction is discussed further in Appendix A.

The eigenproblem for the Hamiltonian (3.5) is separable into a radial and an angular part, as in Eqs. (2.5) and (2.6), provided $g(\beta)=1$ or $1 / \beta^{2}$. The interaction may be evaluated in terms of the Casimir operators (A.3) of the total, core, and fermion five-dimensional angular momentum algebras as

$$
\begin{aligned}
2 \hat{\Lambda} \circ \hat{\Sigma} & =\hat{\Lambda}_{B F} \circ \hat{\Lambda}_{B F}-\hat{\Lambda} \circ \hat{\Lambda}-\hat{\Sigma} \circ \hat{\Sigma} \\
& =\frac{1}{2}\left[C_{2}\left[\operatorname{Spin}_{B F}(5)\right]-C_{2}\left[\operatorname{Spin}_{B}(5)\right]-C_{2}\left[\operatorname{Spin}_{F}(5)\right]\right] .
\end{aligned}
$$

The choice $g(\beta)=1$ yields a Bose-Fermi dynamical symmetry in the usual sense, since

$$
H=C_{2}[\mathrm{E}(5)]+\frac{k}{2}\left[C_{2}\left[\operatorname{Spin}_{B F}(5)\right]-C_{2}\left[\operatorname{Spin}_{B}(5)\right]-C_{2}\left[\operatorname{Spin}_{F}(5)\right]\right]
$$

The interaction $\hat{\Lambda} \circ \hat{\Sigma}$ enforces a five-dimensional angular momentum coupling scheme in which the states have both good SO(5) angular momentum and good total $\operatorname{Spin}_{B F}(5)$ angular momentum. The coupling of the core representation $(\tau, 0)$ and the fermion representation $\left(\frac{1}{2}, \frac{1}{2}\right)$ gives two representations $\left(\tau_{1}, \frac{1}{2}\right)$ 
with $\tau_{1}=\tau \pm 1 / 2$ for the total system, since

$$
(\tau, 0) \otimes\left(\frac{1}{2}, \frac{1}{2}\right)=\left(\tau+\frac{1}{2}, \frac{1}{2}\right) \oplus\left(\tau-\frac{1}{2}, \frac{1}{2}\right) .
$$

The dynamical symmetry is characterized by the subalgebra chain

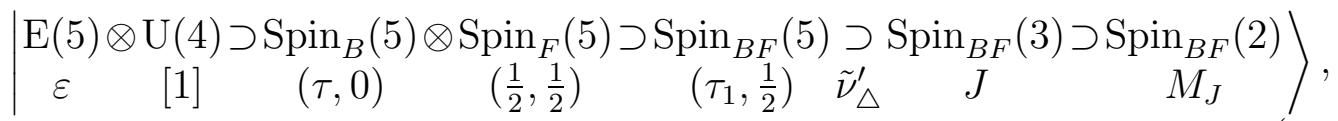

and the eigenstates are members of representations with the quantum numbers indicated. Alternatively, the choice $g(\beta)=1 / \beta^{2}$ was considered in Ref. [20]. In this case, an analytic solution is still possible, much as for the generalized degeneracy breaking interactions considered in Sec. 2.5. However, the states are not eigenstates of $C_{2}[\mathrm{E}(5)]$ and therefore are no longer labeled by the quantum number $\varepsilon$ of $\mathrm{E}(5) \otimes \mathrm{U}(4)$ in (3.9), much as discussed in Sec. 2.5 for the $\mathrm{E}(5)$ case.

The angular states are obtained by the Spin(5) tensor coupling of core states $\left|\tau \tilde{\nu}_{\triangle} L M_{L}\right\rangle \equiv\left|\Psi_{\tau \tilde{\nu} \triangle} L M_{L}(\gamma, \vartheta)\right\rangle$ with single particle states $\left|\frac{3}{2} m_{j}\right\rangle$, according to the $\operatorname{Spin}(5) \supset \operatorname{Spin}(3)$ isoscalar factors [18,65-67]. The coupled state is

$$
\left|\tau \tau_{1} J M_{J}\right\rangle=\sum_{L} \sum_{M_{L}, m_{j}}\left(\begin{array}{cc|c}
(\tau, 0) & \left(\frac{1}{2}, \frac{1}{2}\right) \\
L & \frac{3}{2} & \left(\tau_{1}, \frac{1}{2}\right) \\
J
\end{array}\right)\left(\begin{array}{cc|c}
L & \frac{3}{2} & J \\
M_{L} & m_{j} & M_{J}
\end{array}\right)\left|\tau L M_{L}\right\rangle\left|\frac{3}{2} m_{j}\right\rangle
$$

where multiplicity indices have been suppressed for simplicity. The calculation of the necessary isoscalar factors is discussed further in Appendix B.

If the choice $g(\beta)=1$ is made for the core-fermion coupling in (3.5), the radial equation and its solutions are exactly as in Sec. 2.1. The energies, however, include an additional contribution $2 k\langle\hat{\Lambda} \circ \hat{\Sigma}\rangle$ from the five dimensional spin orbit interaction, where $\langle\hat{\Lambda} \circ \hat{\Sigma}\rangle$ is the eigenvalue of $\hat{\Lambda} \circ \hat{\Sigma}$ acting on the angular state (3.10). The $\operatorname{Spin}(5)$ eigenvalue formula (A.4) gives

$$
\begin{aligned}
2\langle\hat{\Lambda} \circ \hat{\Sigma}\rangle & =\left[\tau_{1}\left(\tau_{1}+3\right)+\frac{3}{4}\right]-[\tau(\tau+3)]-\left[\frac{10}{4}\right] \\
& = \begin{cases}\tau & \tau_{1}=\tau+1 / 2 \\
-(\tau+3) & \tau_{1}=\tau-1 / 2 .\end{cases}
\end{aligned}
$$

If instead the choice $g(\beta)=1 / \beta^{2}$ is made, the contribution of the core-fermion coupling term is absorbed into the separation constant $\Lambda$ in the radial equation (2.6), as

$$
\begin{aligned}
\Lambda & =\tau(\tau+3)+2 k\langle\hat{\Lambda} \circ \hat{\Sigma}\rangle \\
& =\tau(\tau+3)+k\left[\tau_{1}\left(\tau_{1}+3\right)-\tau(\tau+3)-\frac{7}{4}\right] .
\end{aligned}
$$


Table 3

Angular momentum contents of the representations $\left(\tau_{1}, \frac{1}{2}\right)$ of $\operatorname{Spin}(5)$, for $\tau_{1} \leq 7 / 2$.

\begin{tabular}{rllllllll}
\hline$\tau_{1}$ & $J$ & & & & & & \\
\hline $1 / 2$ & $3 / 2$ & & & & & & & \\
$3 / 2$ & $7 / 2$ & $5 / 2$ & $1 / 2$ & & & & & \\
$5 / 2$ & $11 / 2$ & $9 / 2$ & $7 / 2$ & $5 / 2$ & $3 / 2$ & & & \\
$7 / 2$ & $15 / 2$ & $13 / 2$ & $11 / 2$ & $9 / 2$ & $9 / 2$ & $7 / 2$ & $5 / 2$ & $3 / 2$ \\
\hline
\end{tabular}

The eigenvalues and eigenfunctions are given, as in the even-even case, by Eqs. (2.13) and (2.14), but now the order $\nu\left(\tau_{1}, \tau\right)=(\Lambda+9 / 4)^{1 / 2}$ of the Bessel functions is determined by the separation constant as given in (3.21). It therefore depends upon the strength $k$ of the core-fermion coupling.

In practice, introduction of the $1 / \beta^{2}$ dependence does not produce major qualitative changes in the predictions for observables (see Sec. 2.5). Therefore, for the remainder of this article, we shall restrict our attention to the simpler, $\beta$-independent core-fermion coupling $2 k \hat{\Lambda} \circ \hat{\Sigma}$. This choice allows the algebraic classification scheme (3.9) to be retained.

The eigenstates of the $\mathrm{E}(5 \mid 4)$ Hamiltonian are specified by the angular quantum numbers $\left(\tau_{1}, \tau, L\right.$, and $\left.M_{L}\right)$ together with the radial quantum number $\xi(\xi=1,2, \ldots)$. Each value of $\tau_{1}$ yields a multiplet of degenerate states of various angular momenta, according to the $\operatorname{Spin}(5) \supset \operatorname{Spin}(3)$ branching rules [66]. The angular momentum contents of the lowest $\operatorname{Spin}(5)$ representations $\left(\tau_{1}, 1 / 2\right)$ are summarized for convenience in Table 3 . For $\tau_{1} \geq 7 / 2$ the same angular momentum can occur more than once within a $\operatorname{Spin}(5)$ representation, and so a multiplicity index $\tilde{\nu}_{\triangle}^{\prime}$ is required. The eigenstates may thus be denoted $\left|\xi \tau_{1} \tau \tilde{\nu}_{\triangle}^{\prime} L M_{L}\right\rangle$.

The addition of the core-fermion coupling degree of freedom implies that there are two fundamental types of excitation in the $\mathrm{E}(5 \mid 4)$ system: radial excitations, involving changes in the radial quantum number $\xi$, and fivedimensional spin-flip excitations, involving the transition between $\tau_{1}=\tau+1 / 2$ states and $\tau_{1}=\tau-1 / 2$ states. (Note that the spin-flip excitations were not addressed in Ref. [20].) The excitation spectrum for the E(5|4) model (with $k=-1 / 2$ ) is shown in Fig. 10. A concise composite notation for the two types of excitations is obtained by attaching a $\pm \operatorname{sign}$ to the $\xi$ label to indicate $\tau_{1}=\tau \pm 1 / 2$, as suggested in Ref. [32]. That is, the families of levels designated $\xi=1_{+}, 2_{+}, \ldots$ have aligned $\operatorname{Spin}(5)$ coupling $\left(\tau_{1}=\tau+1 / 2\right)$, while those designated $\xi=1_{-}, 2_{-}, \ldots$ have anti-aligned $\operatorname{Spin}(5)$ coupling $\left(\tau_{1}=\tau-1 / 2\right)$.

The evolution of level energies which occurs with changing core-fermion interaction strength $k$ is summarized in Fig. 11. The coupling strength strongly affects the energies of the excited $\left(\xi=1_{-}\right.$and $\left.\xi=2_{+}\right)$families, as discussed further in Sec. 3.3. The value $k=-1 / 2$ will be used as a representative value 


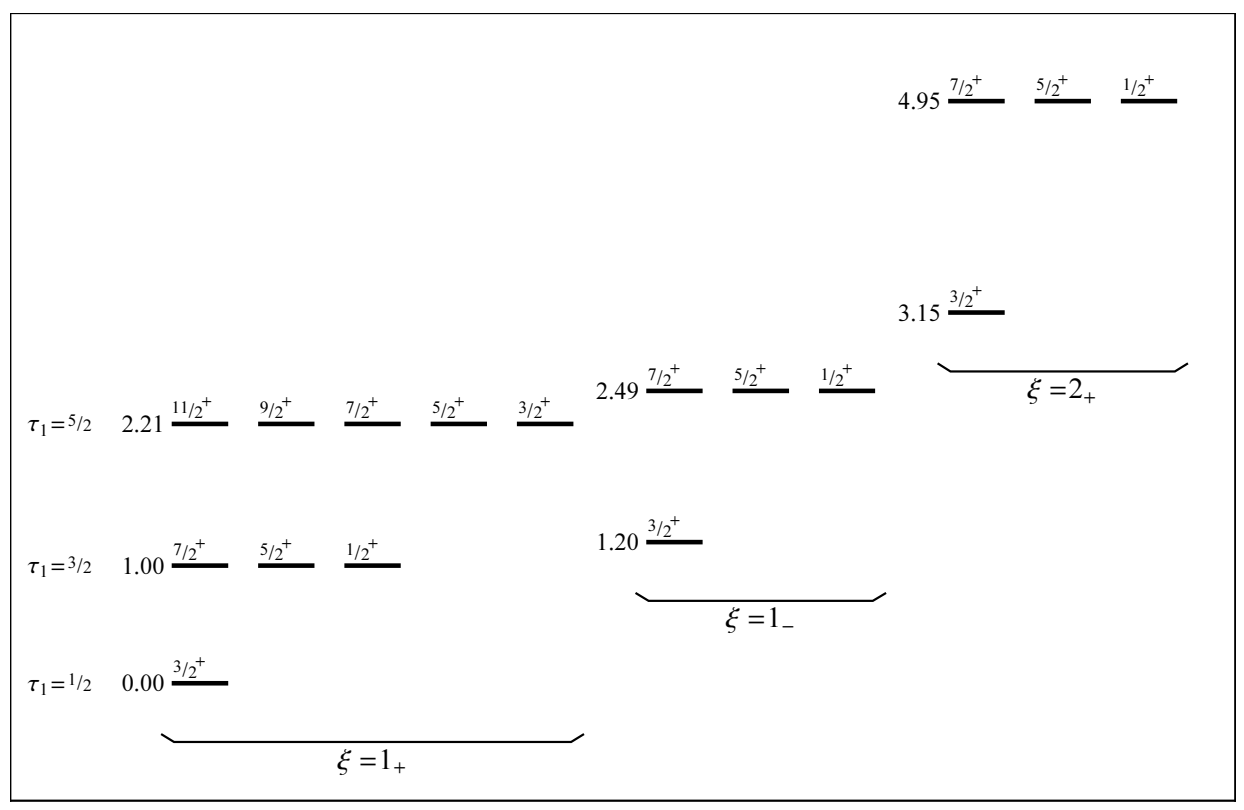

Fig. 10. Level scheme for the $\mathrm{E}(5 \mid 4)$ model for $k=-1 / 2$, showing the excitation energies of the lowest $\tau_{1}$ multiplets of the ground state, spin-flip excited $\left(\xi=1_{-}\right)$, and first radially excited $\left(\xi=2_{+}\right)$families of levels. All energies are normalized to $E\left(7 / 2_{1}^{+}\right)$. The $\xi=1_{-}$family of levels was not included in the corresponding figure of Ref. [20].

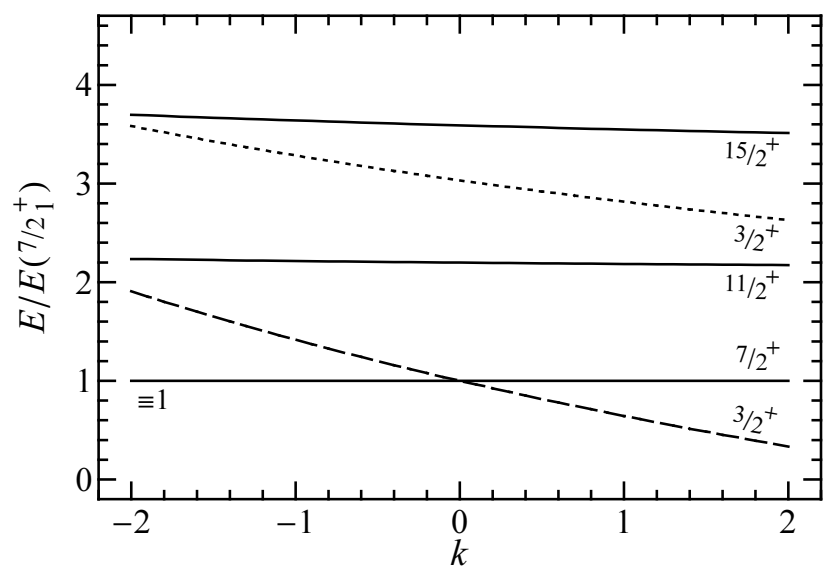

Fig. 11. Evolution of energies as functions of the coupling parameter $k$, for the $\mathrm{E}(5 \mid 4)$ model. Energies are shown for the yrast $7 / 2^{+}, 11 / 2^{+}$, and $15 / 2^{+}$levels (solid curves), the $\xi=1_{-}$family head $3 / 2_{\xi=1_{-}}^{+}$(dashed curve), and the $\xi=2_{+}$family head $3 / 2_{\xi=2_{+}}^{+}$(dotted curve). Energies are normalized to $E\left(7 / 2_{1}^{+}\right)$.

for the remainder of this article, since it yields reasonable excitation energies for use in phenomenological interpretation, e.g., of Ba nuclei. Electromagnetic transition strengths are independent of the coupling strength. 
Table 4

The $\operatorname{Spin}(5) \supset \operatorname{Spin}(3)$ tensor transformation properties of core and fermionic operators. A compound superscript notation such as $(\alpha \times \alpha)^{(2,4)}$ indicates that the Spin(3) tensor products with both angular momenta transform together as a single tensor under $\operatorname{Spin}(5)$.

\begin{tabular}{llll}
\hline Core & Fermionic & $\operatorname{Spin}(5)$ & $\operatorname{Spin}(3)$ \\
\hline$(\alpha \times \alpha)^{(0)}$ & $\left(a^{\dagger} \times \tilde{a}\right)^{(0)}$ & $(0,0)$ & 0 \\
$\alpha, \tilde{\pi}$ & $\left(a^{\dagger} \times \tilde{a}\right)^{(2)}$ & $(1,0)$ & 2 \\
$(\alpha \times \alpha)^{(2,4)}$ & & $(2,0)$ & 2,4 \\
$\hat{\Lambda} \propto(\alpha \times \tilde{\pi})^{(1,3) a}$ & $\hat{\Sigma} \propto\left(a^{\dagger} \times \tilde{a}\right)^{(1,3) b}$ & $(1,1)$ & 1,3 \\
& $a^{\dagger}, \tilde{a}$ & $\left(\frac{1}{2}, \frac{1}{2}\right)$ & $\frac{3}{2}$ \\
\hline
\end{tabular}

The operator $\hat{L}$ is proportional to the component $\hat{\Lambda}^{(1)}$ of this tensor.

${ }^{b}$ The operator $\hat{j}$ is proportional to the component $\hat{\Sigma}^{(1)}$ of this tensor.

\subsection{Electromagnetic transition strengths}

\subsubsection{General properties}

For the coupled odd-mass system, both collective (core) and fermionic contributions are present in the electromagnetic transition strengths. The transition operators of Sec. 2.3 must be extended to contain fermionic terms, so

$$
T=T_{B}+T_{F}
$$

where $T_{B}$ acts only on the core and $T_{F}$ acts only on the fermion. The $\operatorname{Spin}(5)$ and, of course, Spin(3) tensor properties of the operators play an important role in defining selection rules. Selection rules on the quantum numers of both the core $(\tau$ and $L)$ and the coupled system $\left(\tau_{1}\right.$ and $\left.J\right)$ determine the overall pattern of allowed transitions. The tensor properties of the relevant operators are summarized in Table 4.

The problem of computing transition strengths for the coupled $\mathrm{E}(5 \mid 4)$ system can be simplified to that of calculating core matrix elements and fermionic matrix elements separately. First, the $\operatorname{Spin}_{B F}(5)$-coupled states defined in (3.10) must be decomposed in terms of states coupled at the $\operatorname{Spin}_{B F}(3)$ level, using the $\operatorname{Spin}(5) \supset \operatorname{Spin}(3)$ isoscalar factors. Then the usual Racah reduction formulas [54] for angular-momentum coupled systems apply. For a 
core operator $T_{B}^{(\lambda)}$ of multipolarity $\lambda$, this yields

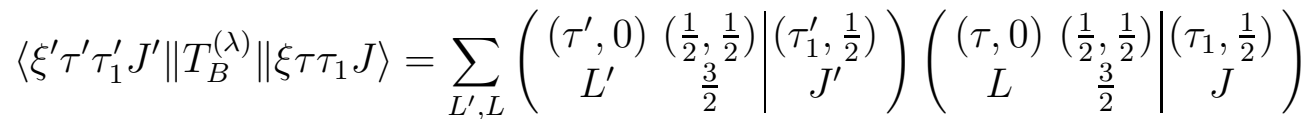

$$
\begin{aligned}
& \times(-)^{L^{\prime}+J+\lambda+3 / 2}\left(2 J^{\prime}+1\right)^{1 / 2}(2 J+1)^{1 / 2}\left\{\begin{array}{ccc}
L^{\prime} & J^{\prime} & \frac{3}{2} \\
J & L & \lambda
\end{array}\right\}\left\langle\xi^{\prime} \tau^{\prime} L^{\prime}\left\|T_{B}^{(\lambda)}\right\| \xi \tau L\right\rangle .
\end{aligned}
$$

Here the core matrix element $\left\langle\xi^{\prime} \tau^{\prime} L^{\prime}\left\|T_{B}^{(\lambda)}\right\| \xi \tau\right\rangle$ is calculated as in Sec. 2.3, though now the Bessel function orders and eigenvalues appearing in the radial integral (2.25) are the E(5|4) values from (3.21). Similarly, for a single particle operator $T_{F}^{(\lambda)}$ of multipolarity $\lambda$,

$$
\begin{aligned}
& \left\langle\xi^{\prime} \tau^{\prime} \tau_{1}^{\prime} J^{\prime}\left\|T_{F}^{(\lambda)}\right\| \xi \tau \tau_{1} J\right\rangle=\sum_{L}\left(\begin{array}{cc|c}
(\tau, 0) & \left(\frac{1}{2}, \frac{1}{2}\right) & \left(\tau_{1}^{\prime}, \frac{1}{2}\right) \\
L & \frac{3}{2} & J^{\prime}
\end{array}\right)\left(\begin{array}{cc|c}
(\tau, 0) & \left(\frac{1}{2}, \frac{1}{2}\right) & \left(\tau_{1}, \frac{1}{2}\right) \\
L & \frac{3}{2} & J
\end{array}\right) \\
& \times(-)^{L+J^{\prime}+\lambda+3 / 2}\left(2 J^{\prime}+1\right)^{1 / 2}(2 J+1)^{1 / 2}\left\{\begin{array}{ccc}
\frac{3}{2} & J^{\prime} & L \\
J & \frac{3}{2} & \lambda
\end{array}\right\} \delta_{\xi^{\prime} \xi} \delta_{\tau^{\prime} \tau}\left\langle\frac{3}{2}\left\|T_{F}^{(\lambda)}\right\| \frac{3}{2}\right\rangle .
\end{aligned}
$$

The fermionic matrix element is given by $\left\langle\frac{3}{2}\left\|\left(a^{\dagger} \times \tilde{a}\right)^{(\lambda)}\right\| \frac{3}{2}\right\rangle=-(2 \lambda+1)^{1 / 2}$.

The angular matrix elements for the various transition operators are common to all problems involving the coupling of a $\gamma$-soft core to a $j=3 / 2$ fermion in the Spin(5) coupling scheme. These generic angular matrix elements are tabulated for reference in Appendix D. Only the radial matrix elements depend upon the specific choice of core potential (the square well in this case) or possible $\beta$ dependence of the core-fermion coupling.

\subsubsection{E2 transitions}

Incorporating the leading fermionic contribution into the $E 2$ operator $(2.21)$ gives

$$
T^{(E 2)}=t_{21} \alpha+t_{22}(\alpha \times \alpha)^{(2)}+t_{22}^{\prime}\left(a^{\dagger} \times \tilde{a}\right)^{(2)} .
$$

The leading order collective term $\alpha$ is a $(1,0)$ tensor operator with respect both to $\mathrm{SO}(5)$, as already noted, and to $\operatorname{Spin}_{B F}(5)$. The former property leads to the selection rule $\Delta \tau= \pm 1$, the latter to the selection rule $\Delta \tau_{1}=0$ or $\pm 1[65,66]$. The $\Delta \tau=1, \Delta \tau_{1}= \pm 1$ transitions connect states in successive $\tau_{1}$ multiplets of the same family of states (e.g., producing transitions within the $\xi=1_{-}$or $\xi=1_{+}$families). The $\Delta \tau=1, \Delta \tau_{1}=0$ transitions instead connect states of a given $\tau_{1}$ multiplet in one family with those in the corresponding $\tau_{1}$ multiplet of the spin-flip excited family (e.g., connecting the $\xi=1_{-}$and $\xi=1_{+}$ families). Intra-multiplet transitions and quadrupole moments are forbidden for the leading-order collective transition operator, as in the even-even case. 


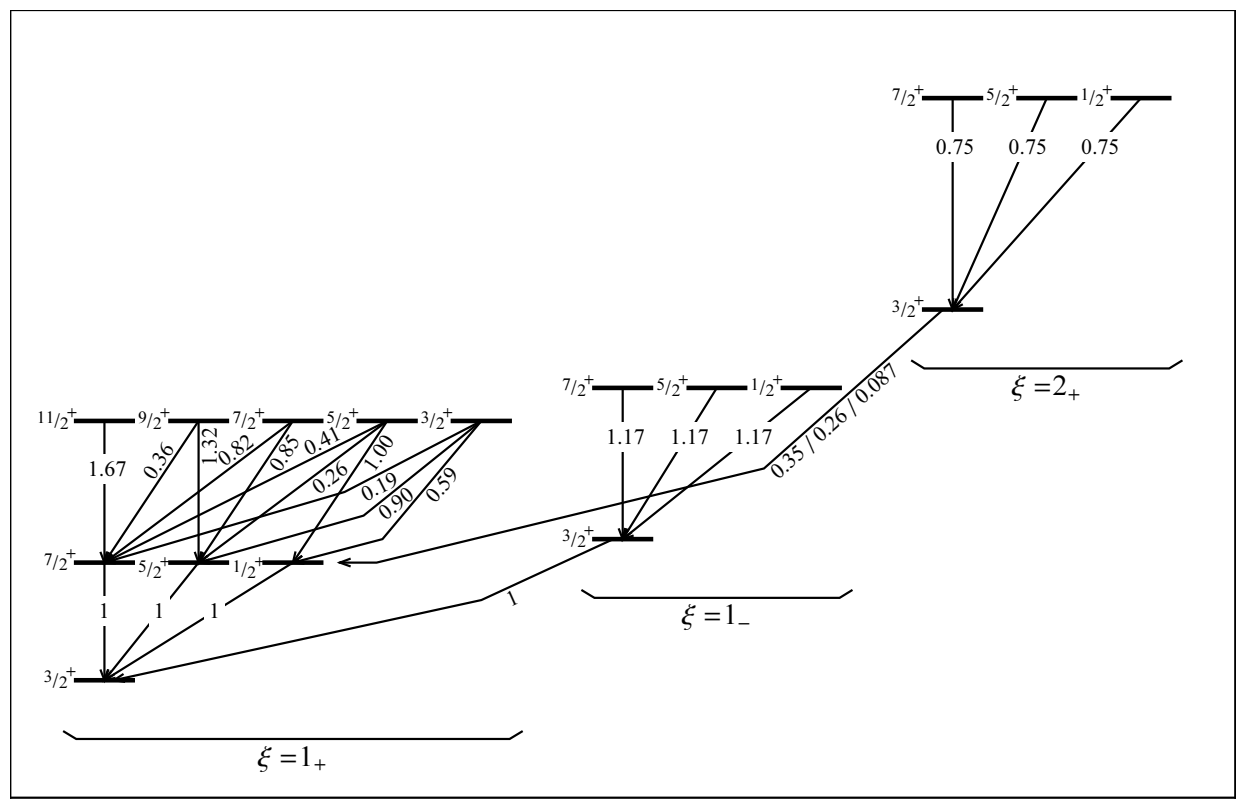

Fig. 12. $B(E 2)$ strengths induced by the operator $t_{21} \alpha$, for the $\mathrm{E}(5 \mid 4)$ model. Transitions obey the selection rules $\Delta \tau= \pm 1$ and $\Delta \tau_{1}=0$ or \pm 1 . All transition strengths are normalized relative to $B\left(E 2 ; 7 / 2_{1}^{+} \rightarrow 3 / 2_{1}^{+}\right)$. For absolute values, strengths should be multiplied by $(0.07453) t_{21}^{2} \beta_{W}^{2}$.

Table 5

Selected $B(E 2)$ and $B(M 1)$ values for transitions involving interference between core and fermionic contributions, for the $\mathrm{E}(5 \mid 4)$ model.

\begin{tabular}{rrrrrrcc}
\hline$\xi$ & $\tau_{1}$ & $J$ & $\xi^{\prime}$ & $\tau_{1}^{\prime}$ & $J^{\prime}$ & $B(E 2)$ & $B(M 1)$ \\
\hline $1_{+}$ & $3 / 2$ & $7 / 2$ & $1_{+}$ & $3 / 2$ & $5 / 2$ & {$\left[(-0.072) t_{22} \beta_{W V}^{2}+(-0.717) t_{22}^{\prime}\right]^{2}$} & {$\left[(-0.293) t_{10}+(-0.414) t_{12}^{\prime}\right]^{2}$} \\
$1_{+}$ & $3 / 2$ & $5 / 2$ & $1_{+}$ & $3 / 2$ & $1 / 2$ & {$\left[(+0.040) t_{22} \beta_{W V}^{2}+(-0.592) t_{22}^{\prime}\right]^{2}$} & \\
$1_{-}$ & $1 / 2$ & $3 / 2$ & $1_{+}$ & $3 / 2$ & $7 / 2$ & {$\left[(+0.042) t_{22} \beta_{W V}^{2}+(-0.632) t_{22}^{\prime}\right]^{2}$} & \\
$1_{-}$ & $1 / 2$ & $3 / 2$ & $1_{+}$ & $3 / 2$ & $5 / 2$ & {$\left[(+0.092) t_{22} \beta_{W V}^{2}+(+0.548) t_{22}^{\prime}\right]^{2}$} & {$\left[(+0.458) t_{10}+(+0.648) t_{12}^{\prime}\right]^{2}$} \\
$1_{-}$ & $1 / 2$ & $3 / 2$ & $1_{+}$ & $3 / 2$ & $1 / 2$ & {$\left[(-0.074) t_{22} \beta_{W}^{2}+(+0.316) t_{22}^{\prime}\right]^{2}$} & {$\left[(-0.387) t_{10}+(-0.548) t_{12}^{\prime}\right]^{2}$} \\
\hline
\end{tabular}

The transition strengths resulting from the leading order operator are shown in Fig. 12.

The second-order collective quadrupole operator $(\alpha \times \alpha)^{(2)}$ induces $\Delta \tau=0$ $\left(\Delta \tau_{1}=0\right.$ or \pm 1$)$ transitions, as shown in Fig. 13(a), and yields nonvanishing quadrupole moments, shown in Fig. 14. The operator also induces $\Delta \tau= \pm 2$ $\left(\Delta \tau_{1}= \pm 1\right.$ or \pm 2$)$ transitions, shown in Fig. 13(b). The fermionic quadrupole operator $\left(a^{\dagger} \times \tilde{a}\right)^{(2)}$ likewise contributes to $\Delta \tau=0\left(\Delta \tau_{1}=0\right.$ or \pm 1$)$ transitions and to the quadrupole moments. Any analysis of transition strengths must therefore address the interference between these terms. The combined strengths for the most experimentally relevant transitions are given in Table 5, and the combined quadrupole moments are given in Table 6. 


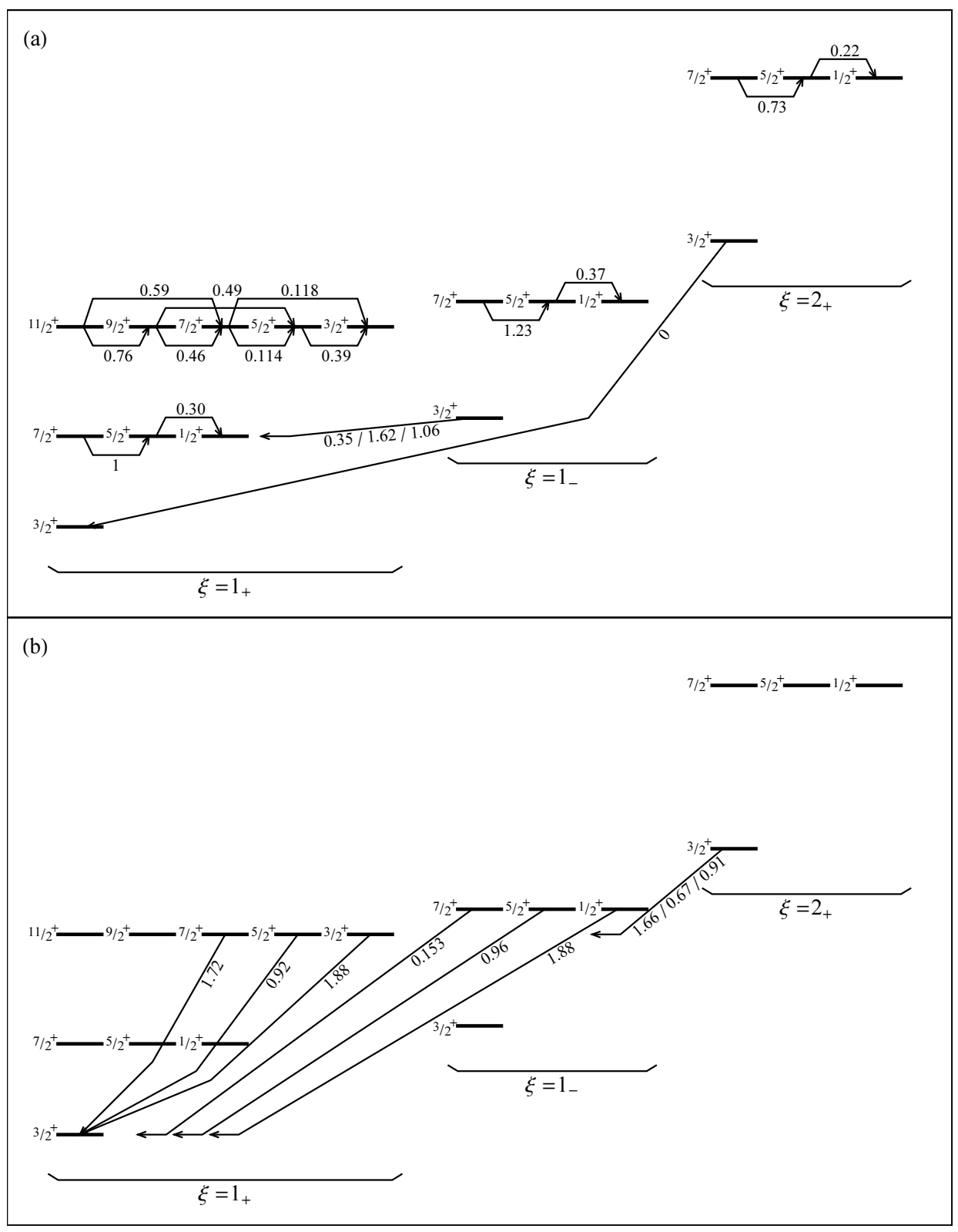

Fig. 13. $B(E 2)$ strengths induced by the operator $t_{22}(\alpha \times \alpha)^{(2)}$, for the $\mathrm{E}(5 \mid 4)$ model: (a) transitions with $\Delta \tau=0$ (and $\Delta \tau_{1}=0$ or \pm 1 ) and (b) transitions with $\Delta \tau= \pm 2$ (and $\Delta \tau_{1}= \pm 1$ or \pm 2 ). All transition strengths are normalized relative to $B\left(E 2 ; 7 / 2_{1}^{+} \rightarrow 5 / 2_{1}^{+}\right)$. For absolute values, transition strengths should be multiplied by $(0.005194) t_{22}^{2} \beta_{W}^{4}$.

\subsubsection{M1 transitions}

The magnetic dipole operator (2.27) with fermionic contribution is

$$
\begin{aligned}
T^{(M 1)} & =t_{10}\left[i(\alpha \times \tilde{\pi})^{(1)}\right]+t_{11}\left[\alpha \times i(\alpha \times \tilde{\pi})^{(1)}\right]^{(1)}+t_{12}^{\prime}\left(a^{\dagger} \times \tilde{a}\right)^{(1)} \\
& =t_{10} \frac{1}{\sqrt{10}} \hat{L}+t_{11} \frac{1}{\sqrt{10}}(\alpha \times \hat{L})^{(1)}-t_{12}^{\prime} \frac{1}{\sqrt{5}} \hat{j}
\end{aligned}
$$




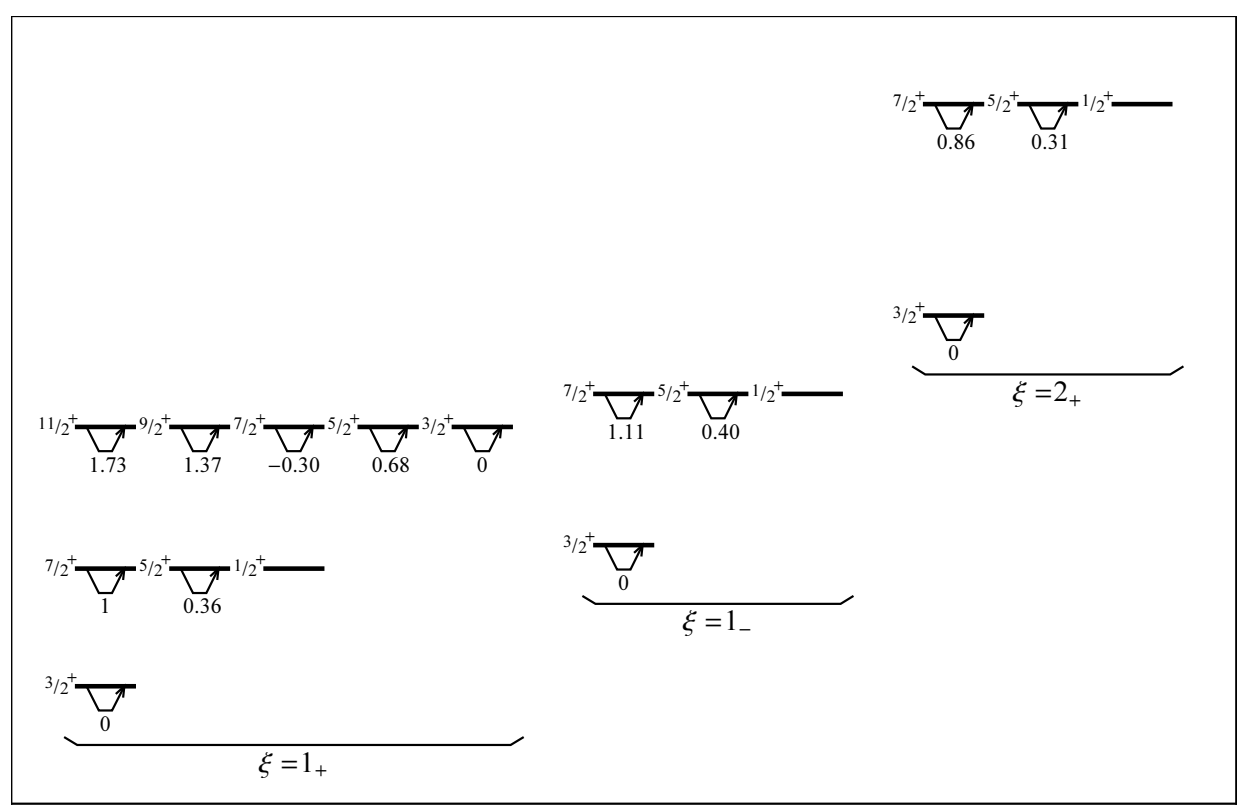

Fig. 14. Quadrupole moments induced by the operator $t_{22}(\alpha \times \alpha)^{(2)}$, for the $\mathrm{E}(5 \mid 4)$ model. All moments are normalized relative to $Q\left(7 / 2_{1}^{+}\right)$. For absolute values, moments should be multiplied by $(0.2124) t_{22} \beta_{W}^{2}$.

Table 6

Selected $Q$ and $\mu$ values, including both core and fermionic contributions, for the $\mathrm{E}(5 \mid 4)$ model.

\begin{tabular}{rrrrr}
\hline$\xi$ & $\tau_{1}$ & $J$ & $Q(J)$ & $\mu(J)$ \\
\hline $1_{+}$ & $1 / 2$ & $3 / 2$ & $(-1.585) t_{22}^{\prime}$ & $(-1.373) t_{12}^{\prime}$ \\
$1_{+}$ & $3 / 2$ & $7 / 2$ & $(+0.212) t_{22} \beta_{W}^{2}+(-1.585) t_{22}^{\prime}$ & $(+1.294) t_{10}+(-1.373) t_{12}^{\prime}$ \\
$1_{+}$ & $3 / 2$ & $5 / 2$ & $(+0.076) t_{22} \beta_{W}^{2}+(+0.226) t_{22}^{\prime}$ & $(+1.017) t_{10}+(-0.850) t_{12}^{\prime}$ \\
$1_{+}$ & $3 / 2$ & $1 / 2$ & & $(+0.647) t_{10}+(+0.458) t_{12}^{\prime}$ \\
$1_{-}$ & $1 / 2$ & $3 / 2$ & $(+0.951) t_{22}^{\prime}$ & $(+0.777) t_{10}+(-0.275) t_{12}^{\prime}$ \\
$2_{+}$ & $1 / 2$ & $3 / 2$ & $(-1.585) t_{22}^{\prime}$ & $(-1.373) t_{12}^{\prime}$ \\
\hline
\end{tabular}

The leading collective term is proportional to the collective angular momentum operator $\hat{L}$, and, similarly, the fermionic term is proportional to the single particle angular momentum operator $\hat{j}$.

Though $\hat{L}$ is a diagonal operator for the even-even system in isolation (Sec 2.3), it is no longer diagonal for the coupled odd-mass system. It can connect any two states which share amplitudes for the same core wave function $|\xi \tau L\rangle$. Transitions induced by $\hat{L}$ obey the selection rules $\Delta \tau=0$ and $\Delta \tau_{1}=0$ or \pm 1 . The transition strengths and dipole moments arising from this leading collective term are shown in Fig. 15.

The fermionic term $\hat{j}$ induces transitions and dipole moments obeying the 


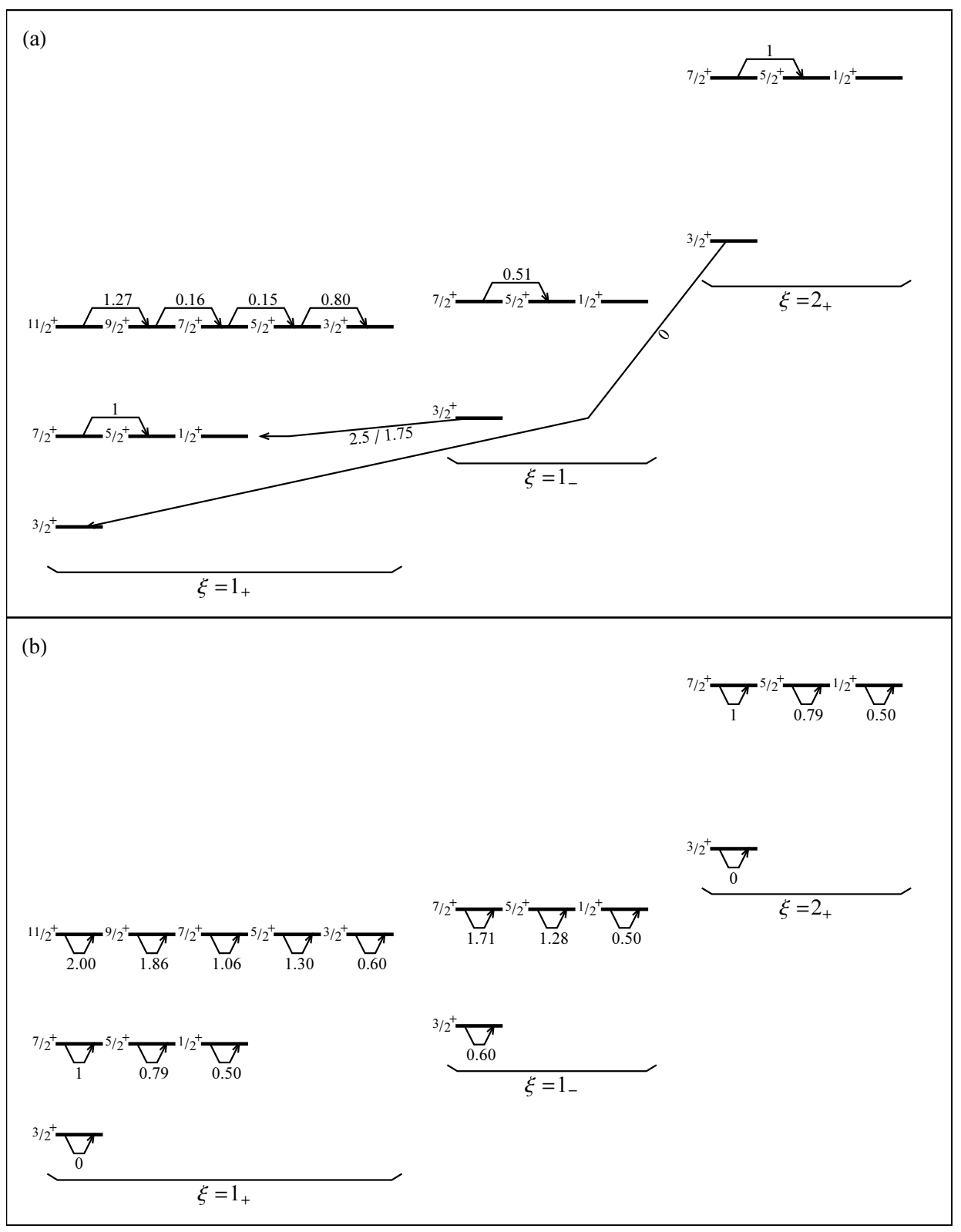

Fig. 15. (a) $B(M 1)$ strengths and (b) dipole moments induced by the operator $t_{10} i(\alpha \times \pi)^{(1)}$, for the $\mathrm{E}(5 \mid 4)$ model. Transitions obey the selection rule $\Delta \tau=0$ and $\Delta \tau_{1}=0$ or \pm 1 . All transition strengths are normalized relative to $B\left(M 1 ; 7 / 2_{1}^{+} \rightarrow 5 / 2_{1}^{+}\right)$and moments relative to $\mu\left(7 / 2_{1}^{+}\right)$. For absolute values, transition strengths should be multiplied by $(0.08571) t_{10}^{2}$ and moments by $(1.294) t_{10}$.

same selection rules as $\hat{L}$, so, in general, interference of the two operators is present. The combination $\hat{J}=\hat{L}+\hat{j}$ is the total angular momentum operator of the coupled system, which is diagonal and has reduced matrix element $[J(J+1)(2 J+1)]^{1 / 2}$. The matrix elements of the two operators $\hat{L}$ and $\hat{j}$ are therefore closely related. In particular, for all off-diagonal matrix elements 
(dipole transitions), $\left\langle\xi^{\prime} \tau^{\prime} \tau_{1}^{\prime} J^{\prime}\|\hat{L}\| \xi \tau \tau_{1} J\right\rangle=-\left\langle\xi^{\prime} \tau^{\prime} \tau_{1}^{\prime} J^{\prime}\|\hat{j}\| \xi \tau \tau_{1} J\right\rangle$. Consequently, although interference of the core and fermionic contributions to $T^{(M 1)}$ affects the overall normalization of the $\Delta \tau=0$ dipole transition strengths, which are proportional to $\left(t_{10}+\sqrt{2} t_{12}^{\prime}\right)^{2}$, it does not affect the relative strengths of different transitions (Table 5). For diagonal matrix elements (dipole moments), interference affects the values in a less trivial fashion (Table 6).

The higher-order collective term $(\alpha \times \hat{L})^{(1)}$ in the dipole operator yields transitions obeying the selection rule $\Delta \tau= \pm 1$. The most experimentally accessible $\Delta \tau= \pm 1$ transitions are those between the various low-lying $\tau_{1}=3 / 2$ and $\tau_{1}=1 / 2$ states. However, the matrix elements of $(\alpha \times \hat{L})^{(1)}$ between these low-lying states all vanish, as a result of the vanishing $2^{+} \leftrightarrow 0^{+}$core transitions seen in Fig. 5. This limits the phenomenological relevance of the higher-order transition operator. Transition strengths can be calculated if needed from the angular matrix elements in Table D.5.

\subsection{Comparison with experimental data}

A comparison of the $\mathrm{E}(5 \mid 4)$ description with experimental data for oddmass nuclei is more difficult than the comparison of the E(5) description with even-even nuclei, because the occurence of other single particle orbitals near the $j=3 / 2$ orbital can considerably perturb the spectrum and electromagnetic transition strengths. Much as $\mathrm{SO}(5)$ symmetry dictates the multiplet structure and branching patterns for the E(5) predictions (Sec. 2.4), the basic form of the $\mathrm{E}(5 \mid 4)$ predictions is a consequence of the Spin(5) coupling scheme, rather than of the $\mathrm{E}(5 \mid 4)$ square well potential per se. Therefore, we concentrate here upon those distinguishing observables which vary along the $\mathrm{U}(5)-\mathrm{SO}(6)$ transition.

However, the Spin(5) coupling scheme is itself an important aspect of the model to test. In the IBFM, the U(5) symmetry limit is treated in the Spin(5) coupling scheme, but the $\mathrm{SO}(6)$ limit is instead treated in a Spin(6) coupling scheme [66]. While the Spin(5) coupled states considered in the present work are $\tau$ eigenstates, the $\operatorname{Spin}(6)$ coupled states are near-equal $\tau=\tau_{1} \pm 1 / 2$ admixtures. The leading order $E 2$ operator $(\Delta \tau= \pm 1)$ therefore yields nonvanishing matrix elements between members of a $\tau_{1}$ multiplet (see, e.g., Fig. 5 of Ref. [66]), by connecting the $\tau=\tau_{1} \pm 1 / 2$ components of the different states.

The observables most sensitive to the $\mathrm{U}(5)-\mathrm{SO}(6)$ structural transition are the yrast properties and the energies and decay properties of the spinflip $\left(\xi=1_{-}\right)$and radial $\left(\xi=2_{+}\right)$excitations. The yrast energies and $B(E 2)$ strengths are shown in Fig. 16. Note that there is a moderate dependence of the yrast energies [Fig. 16(a)] upon the core-fermion coupling strength $k$. 


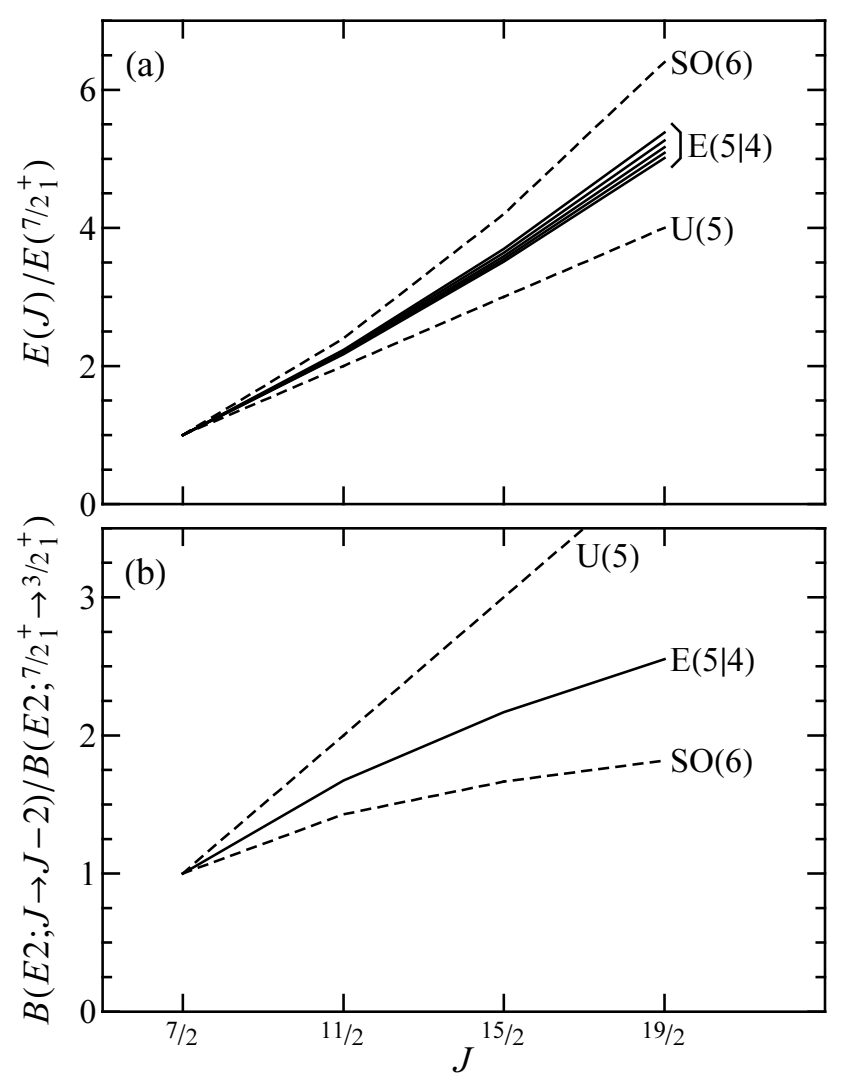

Fig. 16. (a) Energies and (b) $B(E 2)$ strengths as functions of angular momentum, for the yrast states $\left(\xi=1, \tau_{1}=\tau+1 / 2, J=2 \tau_{1}+1 / 2\right)$ in the $\mathrm{E}(5 \mid 4)$ model. Energies are normalized to $E\left(3 / 2_{1}^{+}\right)$and $B(E 2)$ strengths to $B\left(E 2 ; 7 / 2_{1}^{+} \rightarrow 3 / 2_{1}^{+}\right)$. The energies depend upon the coupling constant $k$ and are shown here for $k=-2$, $-1,0,+1$, and +2 (solid curves, uppermost to lowermost). Values for the $U(5)$ and $\mathrm{SO}(6)$ dynamical symmetries in the large boson number limit are shown for comparison (dashed curves).

Since $2\langle\hat{\Lambda} \circ \hat{\Sigma}\rangle=\tau$ for the ground state family, a positive contribution from this term $(k>0)$ produces a more linear [U(5)-like] yrast energy curve. The spin-flip excitation energy is, naturally, strongly dependent upon the strength of the five-dimensional spin-orbit interaction (Fig. 11). The spin-flip excitation varies from near degeneracy with the ground state to near degeneracy with the $\tau_{1}=5 / 2$ multiplet over the range of $k$ values considered $(-2 \leq k \leq+2)$. It therefore serves as the main basis for determining the coupling strength $k$ within the model. For $k=0$, the weak-coupling limit is recovered, and the $3 / 2^{+}$state $\left(\tau_{1}=1 / 2\right)$ of the $\xi=1_{-}$excitation forms a degenerate multiplet with the $7 / 2^{+}, 5 / 2^{+}$, and $1 / 2^{+}$states $\left(\tau_{1}=3 / 2\right)$ of the $\xi=1_{+}$ground state family. The energy of the radial excitation is somewhat less sensitive to $k$, varying from $\sim 2.6$ to 3.6 times the ground state $\tau_{1}=3 / 2$ multiplet energy over the same range of $k$ values (Fig. 11). 
Only limited data are presently available for comparison with the E(5|4) predictions. The nucleus ${ }^{135} \mathrm{Ba}$ has been considered as a candidate for description by the $\mathrm{E}(5 \mid 4)$ model in Ref. [32]. Another possible candidate is the nucleus ${ }^{63} \mathrm{Cu}$, previously considered as a good example of Spin(5) symmetry for a $j=3 / 2$ particle coupled to a $\mathrm{U}(5)$ boson core [68]. The available energies and transition strengths are consistent with either a $\mathrm{U}(5)$ interpretation or an $\mathrm{E}(5 \mid 4)$ interpretation, since, unfortunately, they test only the common Spin(5) symmetry. In order to assess whether or not E(5|4) might be a better description, one must experimentally determine the properties of the states in the $\xi=2_{+}$family, in particular the energy of the $J=3 / 2\left(\tau_{1}=1 / 2\right)$ state and its $E 2$ transition strengths to the members of the $\tau_{1}=3 / 2$ multiplet of the ground state family.

\subsection{Generalizations of the $\mathrm{E}(5 \mid 4)$ description}

For odd-mass nuclei, the energy splitting of the Spin(5) multiplets is typically much more dramatic than the splitting of $\mathrm{SO}(5)$ multiplets discussed in Sec. 2.5. It must therefore be considered carefully in the analysis, and it cannot be separated from the determination of $k$, since any significant spindependent perturbation to the energies also substantially modifies the yrast and excited family energy ratios considered above. The generalized dynamical symmetry Hamiltonian for the subalgebra chain (3.9) is of the form

$$
\begin{aligned}
H=C_{2}[\mathrm{E}(5)]+c^{\prime \prime} C_{2}\left[\operatorname{Spin}_{B}(5)\right] & +c^{\prime \prime \prime} C_{2}\left[\operatorname{Spin}_{F}(5)\right] \\
& +c C_{2}\left[\operatorname{Spin}_{B F}(5)\right]+c^{\prime} C_{2}\left[\operatorname{Spin}_{B F}(3)\right]
\end{aligned}
$$

or, equivalently, since $\hat{\Lambda} \circ \hat{\Sigma}$ is just a linear combination of $C_{2}\left[\operatorname{Spin}_{B F}(5)\right]$, $C_{2}\left[\operatorname{Spin}_{B}(5)\right]$, and $C_{2}\left[\operatorname{Spin}_{F}(5)\right]$

$$
H=\tilde{\pi} \cdot \tilde{\pi}+V(\beta)+2 k \hat{\Lambda} \circ \hat{\Sigma}+k^{\prime \prime} \hat{\Lambda} \circ \hat{\Lambda}+k^{\prime \prime \prime} \hat{\Sigma} \circ \hat{\Sigma}+k^{\prime} \hat{J} \cdot \hat{J}
$$

These coupling terms are all diagonal in the states described by (3.9), and they affect only the energies, not eigenstates. A level scheme for representative values of $k$ and $k^{\prime}$ is shown in Fig. 17.

Alternatively, as in Sec. 2.5, a limited variety of more general $\beta$ dependences can be included in the degeneracy breaking terms while still preserving the analytically solvable radial equation, e.g.,

$$
H=\tilde{\pi} \cdot \tilde{\pi}+V(\beta)+2 k \frac{\hat{\Lambda} \circ \hat{\Sigma}}{\beta^{2}}+k^{\prime \prime} \frac{\hat{\Lambda} \circ \hat{\Lambda}}{\beta^{2}}+k^{\prime \prime \prime} \frac{\hat{\Sigma} \circ \hat{\Sigma}}{\beta^{2}}+k^{\prime} \frac{\hat{J} \cdot \hat{J}}{\beta^{2}} .
$$

For angular momentum degeneracy breaking, we need only consider $k^{\prime}$ (and 


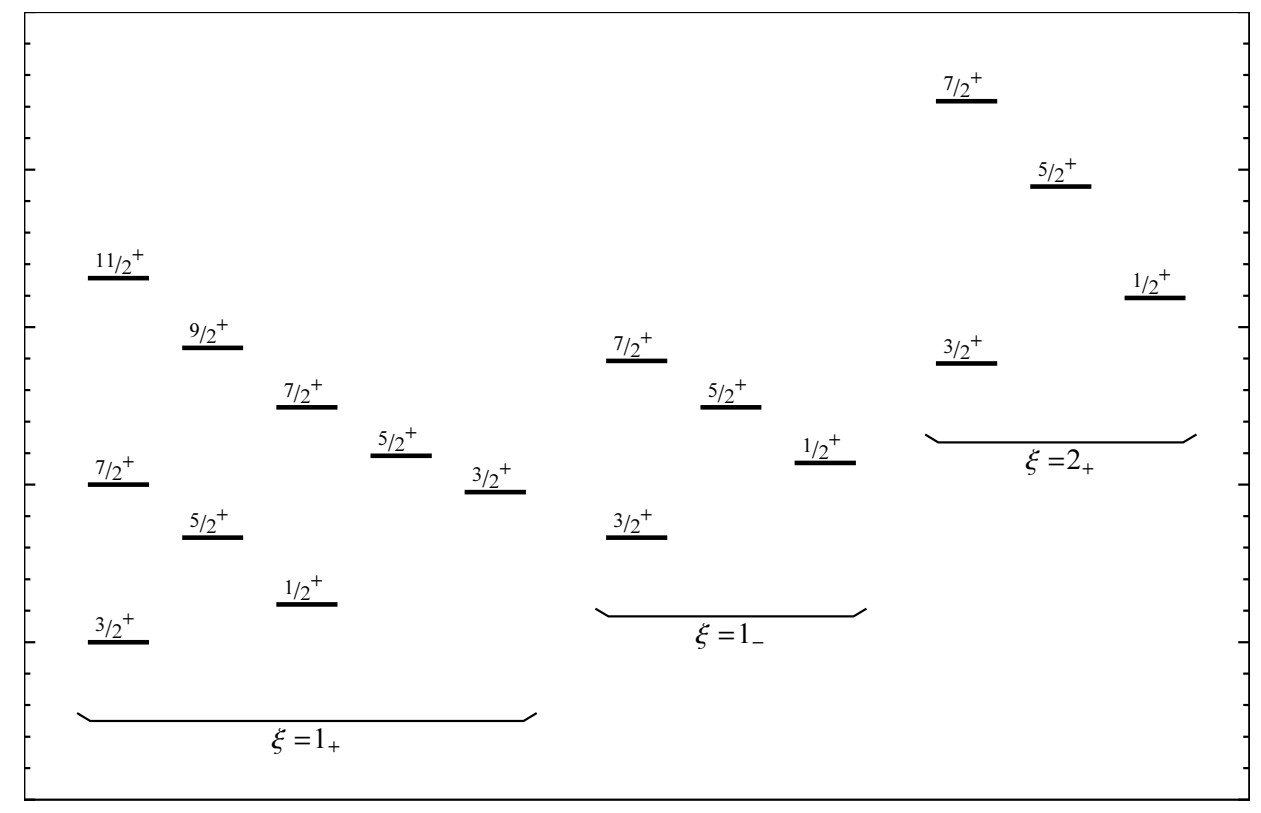

Fig. 17. Level scheme for the E(5|4) model, with core-fermion coupling parameter value $k=-1 / 2$ and angular momentum degeneracy breaking parameter value $k^{\prime}=1 / 2$. All energies are normalized to $E\left(7 / 2_{1}^{+}\right)$.

k) nonzero, giving

$$
\Lambda=\tau(\tau+3)+k\left[\tau_{1}\left(\tau_{1}+3\right)-\tau(\tau+3)-\frac{7}{4}\right]+k^{\prime} J(J+1) .
$$

\section{Conclusion}

In this article, we have considered solutions to the Bohr equation with a square well potential $[\mathrm{E}(5)]$ and for the equation obtained by coupling this to an additional fermion by a five-dimensional spin-orbit interaction [E(5|4)]. These are examples of new classes of dynamical symmetry and Bose-Fermi dynamical symmetry. These symmetries are useful descriptions of even-even and odd-mass nuclei near the critical point of the spherical $[\mathrm{U}(5)]$ to $\gamma$-unstable $[\mathrm{SO}(6)]$ phase transition in nuclei.

The results described here provide benchmarks for nuclei near the critical point of the $\mathrm{U}(5)-\mathrm{SO}(6)$ phase transition and can be used as a basis for comparison with experiment. However, since the Hamiltonian exhibits SO(5) symmetry (for even-even nuclei) or $\operatorname{Spin}_{B F}(5)$ symmetry (for odd-mass nuclei) throughout this transition, in either the algebraic or geometric formulations of the problem, many observables are relatively insensitive to the transition. This makes comparison with experiment challenging. Nonetheless, nuclei with properties close to those of the $\mathrm{E}(5)$ predictions have been identified [21- 
$23,25,26]$, and preliminary comparisons have been carried out for the $\mathrm{E}(5 \mid 4)$ description [32].

The approach discussed here may be extended in several directions. The situation considered in Sec. 3 , of coupling to a $j=3 / 2$ particle, is relevant only to a restricted set of nuclei. Beyond this, the more general possible combinations of angular momenta for the fermion orbitals must also be considered. The case of coupling to orbitals with $j=1 / 2,3 / 2$, and $5 / 2$, termed $\mathrm{E}(5 \mid 12)$, has been treated by Alonso, Arias, and Vitturi [69]. Another important possibility to consider is the embedding of the present Bose-Fermi dynamical symmetry $[\mathrm{E}(5) \otimes \mathrm{U}(4)]$ within a supersymmetry. Within the differential formulation, the related problem of a harmonic oscillator with spin-orbit interaction has already been explored (in three dimensions) by Balantekin [70]. In this case, an $\operatorname{OSp}(1 \mid 2)$ supersymmetry is present. A problem of great phenomenological relevance is the extension of the present approach to the transition between spherical $[\mathrm{U}(5)]$ and axially-symmetric deformed [SU(3)] structure. While the problem can be solved by numerical diagonalization of the Bohr Hamiltonian [53], any analytic solution appears to require substantial approximations [71]. Finally, the general concept of a dynamical symmetry based on the square well potential, for application near the critical point of a phase transition, may be applied to a broad variety of systems other than quadrupole deformed nuclei. The pairing phase transition [72] and the linear-bent phase transition in molecules $[73,74]$ are two examples.

\section{Acknowledgements}

This work was supported in part by the US DOE under grant DE-FG0291ER-40608. We wish to thank R. F. Casten and N. V. Zamfir for providing the first evidence for E(5) structure and M. S. Fetea and collaborators for performing the first comparison of $\mathrm{E}(5 \mid 4)$ with experimental data. Discussions with A. Vitturi, J. M. Arias, and R. Bijker are gratefully acknowledged.

\section{A Algebraic properties and the quadrupole-quadrupole core- fermion interaction}

In this appendix, we establish the relationship between the quadrupolequadrupole core-fermion interaction used in interacting boson fermion model (IBFM) studies [18] and the five-dimensional spin-orbit interaction $\hat{\Lambda} \circ \hat{\Sigma}$ used in the present $\mathrm{E}(5 \mid 4)$ analysis. This is especially of interest to provide grounds

for comparison with the recent study of the $\mathrm{U}(5)-\mathrm{SO}(6)$ transition in the 
IBFM by Alonso et al. [31]. In the process, the basic definitions and relations are established for several algebraic quantities used in the main text.

First, let us review the algebraic properties of the Spin(6) algebra and its subalgebras in Racah form $[3,18,65,66]$. The bosonic $\mathrm{SO}(6)$ algebra and fermionic $\mathrm{SU}(4)$ algebra are isomorphic, so we adopt the more uniform notation $\operatorname{Spin}_{B}(6) \equiv \mathrm{SO}(6)$ and $\operatorname{Spin}_{F}(6) \equiv \mathrm{SU}(4)$. The commutation relations of these algebras are brought into identical form if the generators are normalized as

$$
\begin{aligned}
B^{(1)} & =\left(d^{\dagger} \times \tilde{d}\right)^{(1)} & A^{(1)} & =-\frac{1}{\sqrt{2}}\left(a^{\dagger} \times \tilde{a}\right)^{(1)} \\
B^{(2)} & =\left(s^{\dagger} \times \tilde{d}+d^{\dagger} \times \tilde{s}\right)^{(2)} & A^{(2)} & =\left(a^{\dagger} \times \tilde{a}\right)^{(2)} \\
B^{(3)} & =\left(d^{\dagger} \times \tilde{d}\right)^{(3)} & A^{(3)} & =\frac{1}{\sqrt{2}}\left(a^{\dagger} \times \tilde{a}\right)^{(3)} .
\end{aligned}
$$

The IBFM quadrupole operators are the quadrupole generators, $\hat{Q}_{B} \equiv B^{(2)}$ and $\hat{Q}_{F} \equiv A^{(2)}$. The algebra $\operatorname{Spin}_{B F}(6)$ has generators

$$
G^{(\lambda)}=B^{(\lambda)}+A^{(\lambda)} .
$$

The subalgebras $\operatorname{Spin}_{B}(5) \equiv \mathrm{SO}(5), \operatorname{Spin}_{F}(5) \equiv \mathrm{Sp}(4)$, and $\operatorname{Spin}_{B F}(5)$ are obtained by omitting the quadrupole generators $B^{(2)}, A^{(2)}$, and $G^{(2)}$. The subalgebras $\operatorname{Spin}_{B}(3) \equiv \mathrm{SO}(3), \operatorname{Spin}_{F}(3) \equiv \mathrm{SU}(2)$, and $\operatorname{Spin}_{B F}(3)$ are obtained by further omitting the octupole generators $B^{(3)}, A^{(3)}$, and $G^{(3)}$. The Casimir operators for the algebras are

$$
\begin{aligned}
C_{2}[\operatorname{Spin}(6)] & =2 G^{(2)} \cdot G^{(2)}+4\left[G^{(1)} \cdot G^{(1)}+G^{(3)} \cdot G^{(3)}\right] \\
& =2 G^{(2)} \cdot G^{(2)}+4 G \circ G \\
C_{2}[\operatorname{Spin}(5)] & =4\left[G^{(1)} \cdot G^{(1)}+G^{(3)} \cdot G^{(3)}\right] \\
& =4 G \circ G \\
C_{2}[\operatorname{Spin}(3)] & =20 G^{(1)} \cdot G^{(1)},
\end{aligned}
$$

where $G$ here generically represents the generator $B, A$, or $G$ as appropriate for $\operatorname{Spin}_{B}(n), \operatorname{Spin}_{F}(n)$, or $\operatorname{Spin}_{B F}(n)$. The eigenvalues of these Casimir operators are

$$
\begin{aligned}
\left\langle C_{2}[\operatorname{Spin}(6)]\right\rangle_{\left(\sigma_{1}, \sigma_{2}, \sigma_{3}\right)} & =2\left[\sigma_{1}\left(\sigma_{1}+4\right)+\sigma_{2}\left(\sigma_{2}+2\right)+\sigma_{3}^{2}\right] \\
\left\langle C_{2}[\operatorname{Spin}(5)]\right\rangle_{\left(\tau_{1}, \tau_{2}\right)} & =2\left[\tau_{1}\left(\tau_{1}+3\right)+\tau_{2}\left(\tau_{2}+1\right)\right] \\
\left\langle C_{2}[\operatorname{Spin}(3)]\right\rangle_{(J)} & =2[J(J+1)] .
\end{aligned}
$$

Comparing (A.3) and (A.4), we see that the conventional normalizations of the three-dimensional angular momentum operators are obtained by setting $\hat{L} \equiv \sqrt{10} B^{(1)}$ and $\hat{j} \equiv \sqrt{10} A^{(1)}$, so $\langle\hat{L} \cdot \hat{L}\rangle=L(L+1)$ and $\langle\hat{j} \cdot \hat{j}\rangle=j(j+1)$. Similarly, chosing the normalizations of the five-dimensional angular momentum operators as $\hat{\Lambda}^{(\lambda)} \equiv \sqrt{2} B^{(\lambda)}$ and $\hat{\Sigma}^{(\lambda)} \equiv \sqrt{2} A^{(\lambda)}$ yields $\langle\hat{\Lambda} \circ \hat{\Lambda}\rangle=\tau(\tau+3)$ and $\langle\hat{\Sigma} \circ \hat{\Sigma}\rangle=\tau_{1}\left(\tau_{1}+3\right)+\tau_{2}\left(\tau_{2}+1\right)$. 
Let us now return to the core-fermion interaction. The spin-orbit interaction $\hat{\Lambda} \circ \hat{\Sigma}$ only involves Spin(5) Casimir operators, as shown in (3.6). The quadrupole-quadrupole interaction of the IBFM is, by the above definitions,

$$
\hat{Q}_{B} \cdot \hat{Q}_{F}=\frac{1}{2}\left[G^{(2)} \cdot G^{(2)}-B^{(2)} \cdot B^{(2)}-A^{(2)} \cdot A^{(2)}\right],
$$

Adding to this the five-dimensional spin-orbit interaction

$$
\hat{\Lambda} \circ \hat{\Sigma}=G \circ G-B \circ B-A \circ A,
$$

and recognizing the Spin(6) Casimir operators (A.3), we have

$$
\hat{Q}_{B} \cdot \hat{Q}_{F}+\hat{\Lambda} \circ \hat{\Sigma}=\frac{1}{4}\left[C_{2}\left[\operatorname{Spin}_{B F}(6)\right]-C_{2}\left[\operatorname{Spin}_{B}(6)\right]-C_{2}\left[\operatorname{Spin}_{F}(6)\right]\right] .
$$

Both $\hat{Q}_{B} \cdot \hat{Q}_{F}$ and $\hat{\Lambda} \circ \hat{\Sigma}$ are $\operatorname{Spin}_{B F}(5)$ scalars, so both yield degenerate $\operatorname{Spin}(5)$ multiplets of levels (with good $\tau_{1}$ ) when used as the core-fermion interaction in the Hamiltonian. However, they differ in physical content in an important fashion. The interaction $\hat{\Lambda} \circ \hat{\Sigma}$ induces a pure Spin(5) coupling scheme (where the levels have good $\tau$ as well as $\tau_{1}$ ). The interaction $\hat{Q}_{B} \cdot \hat{Q}_{F}$ additionally contains Spin(6) Casimir operators, as seen from (A.7), so it tends to induce a hybrid of the Spin(5) and Spin(6) coupling schemes (admixed $\tau=\tau_{1} \pm 1 / 2$ ). [This may loosely be interpreted as mixing the spin-aligned and spin-flip states. However, such a simple two-state mixing description strictly holds only in the $\mathrm{SO}(6)$ limit.] Consequently, IBFM predictions obtained with the $\hat{Q}_{B} \cdot \hat{Q}_{F}$ interaction do not exhibit the $\Delta \tau$ selection rules encountered in the $\mathrm{E}(5 \mid 4)$ predictions. This leads to significant qualitative differences between the results of Ref. [31] and the present $\mathrm{E}(5 \mid 4)$ predictions.

\section{B $\quad \operatorname{Spin}(5) \supset \operatorname{Spin}(3)$ isoscalar factors}

The calculation of electromagnetic transition observables for the $\mathrm{E}(5 \mid 4)$ model (Sec. 3) is heavily dependent upon the calculation of the $\operatorname{Spin}(5) \supset$ Spin(3) isoscalar factors involved in the construction of the coupled state core-fermion state (3.10). While the relevant isoscalar factors have been encountered before on several occasions $[18,65-67]$ in the context of the IBFM, considerable phase differences are present among the reported values. In this appendix, we briefly clarify the phase degrees of freedom present in the determination of the $\operatorname{Spin}(5) \supset \operatorname{Spin}(3)$ isoscalar factors and establish a consistent set applicable to the geometric framework for the $\mathrm{E}(5 \mid 4)$ calculations.

The symmetric representations $(\tau, 0)$ of $\operatorname{Spin}_{B}(5)$ and the spinor fundamental representation $\left(\frac{1}{2}, \frac{1}{2}\right)$ of $\operatorname{Spin}_{F}(5)$ are coupled to yield representations 
$\left(\tau \pm \frac{1}{2}, \frac{1}{2}\right)$ of $\operatorname{Spin}_{B F}(5)$ according to the $\operatorname{Spin}(5) \supset \operatorname{Spin}(3)$ isoscalar factors

$$
\left(\begin{array}{cc|c}
(\tau, 0) & \left(\frac{1}{2}, \frac{1}{2}\right) & \left(\begin{array}{c}
(\tau, \\
\tau_{1}
\end{array}\right) \\
\tilde{\nu}_{\triangle} L & \frac{3}{2} & \begin{array}{c}
2 \\
\tilde{\nu}_{\triangle}^{\prime} J
\end{array}
\end{array}\right)
$$

where $\tau_{1}=\tau \pm 1 / 2$. The multiplicity indices $\tilde{\nu}_{\triangle}$ and $\tilde{\nu}_{\triangle}^{\prime}$ are only necessary for $\tau \geq 6$ and $\tau_{1} \geq 7 / 2$, respectively, and so they are suppressed in (3.10) and in the following discussion.

The isoscalar factors are usually obtained $[18,65-67]$ by embedding the Spin(5) algebras within Spin(6) algebras, as outlined in Appendix A, and applying Racah's method. The matrix element of $C_{2}\left[\operatorname{Spin}_{B F}(6)\right]=2 G^{(2)}$. $G^{(2)}+C_{2}[\operatorname{Spin}(5)]$ between a coupled state and an uncoupled state can be evaluated in two different ways: directly by using the eigenvalue formula (A.4) for $C_{2}[\operatorname{Spin}(6)]$ or indirectly by evaluating the matrix element of $G^{(2)} \cdot G^{(2)}$ and using the eigenvalue formula for $C_{2}[\operatorname{Spin}(5)]$. Equating the results obtained by these two methods yields a system of equations involving the isoscalar factors, the core matrix elements $\left\langle\tau L\left\|B^{(2)}\right\| \tau^{\prime} L^{\prime}\right\rangle$, and the trivial fermionic matrix element $\left\langle\frac{3}{2}\left\|A^{(2)}\right\| \frac{3}{2}\right\rangle=-\sqrt{5}$ (see Refs. [18,65-67] for details). Namely, isoscalar factors involving the same values of $\tau_{1}$ and $J$ but different values of $\tau$ and $L$ are related by

$$
\begin{aligned}
0=\left(\begin{array}{cc|c}
(\tau, 0) & \left(\frac{1}{2}, \frac{1}{2}\right) \\
L & \frac{3}{2} & \left(\tau_{1}, \frac{1}{2}\right) \\
J
\end{array}\right)-2 \sqrt{5} \sum_{L^{\prime}}(-)^{3 / 2+L^{\prime}+J}\left\{\begin{array}{ccc}
L & \frac{3}{2} & J \\
\frac{3}{2} & L^{\prime} & 2
\end{array}\right\}\left\langle\tau L\left\|B^{(2)}\right\| \tau^{\prime} L^{\prime}\right\rangle \\
\times\left(\begin{array}{ccc}
\left(\tau^{\prime}, 0\right) & \left(\frac{1}{2}, \frac{1}{2}\right) \\
L^{\prime} & \frac{3}{2} & J
\end{array}\right), \quad\left(\tau_{1}, \frac{1}{2}\right) \\
J .2)
\end{aligned}
$$

where $\tau^{\prime} \equiv \tau_{1} \mp 1 / 2$ when $\tau=\tau_{1} \pm 1 / 2$. The full system is obtained by allowing $\tau$ to range over the values $\tau_{1} \pm 1 / 2$ and $L$ to range over the angular momentum values contained in the $\mathrm{SO}(5)$ representation $(\tau, 0)$.

All isoscalar factors of the same $\tau_{1}$ and $J$ are found simultanously, to within an overall normalization factor and sign (or phase), by solution of the linear homogeneous system of equations given by (B.2). This is equivalent to solution for the null vector of the coefficient matrix of the system, which is easily carried out by Gaussian elimination. The normalization of the family of isoscalar factors sharing the same $\tau_{1}$ and $J$ values follows from the condition

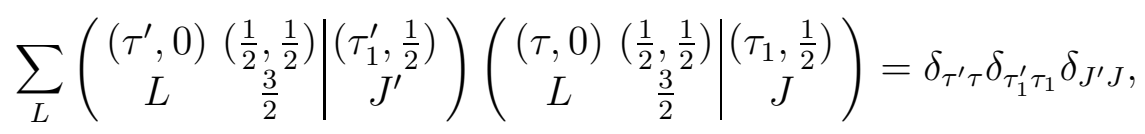

but the overall sign is arbitrary, corresponding to the freedom in defining the sign of the coupled state $\left|\tau \tau_{1} J\right\rangle$ in (3.10).

The relative signs of isoscalar factors sharing the same $\tau_{1}$ and $J$ values are in principle fixed by the system (B.2). However, comparison of the isoscalar 
factors computed in Refs. [18, 65-67] shows apparent sign discrepancies even among sets of isoscalar factors sharing the same $\tau_{1}$ and $J$. The differences arise from differing signs for the matrix elements of $B^{(2)}=\left(s^{\dagger} \times \tilde{d}+d^{\dagger} \times \tilde{s}\right)^{(2)}$ appearing in (B.2). These in turn follow from the phase freedom in defining the $\operatorname{Spin}_{B}(5)$ basis states $|\tau L\rangle$ [42] and, ultimately, from the arbitrary phases of the $\mathrm{U}(5)$ basis states used in defining the matrix elements of $d^{\dagger}$, i.e., $a_{L^{\prime} L}\left(n_{d}\right)[34,42]$. Physical quantities, such as transition matrix elements, for the coupled system are calculated correctly only if the same phase convention for the $\operatorname{Spin}_{B}(5)$ basis states is used consistently both in the construction of the isoscalar factors and in the calculation of physical quantities. Consequently, the isoscalar factors for use in the present work cannot be taken verbatim from any of the Refs. [18,65-67] but rather must be recalculated with phases consistent with the present core $\operatorname{Spin}_{B}(5)$ basis functions, the $\Psi_{\tau \tilde{\nu}_{\triangle} L M_{L}}(\gamma, \vartheta)$.

The form of (B.2) appropriate to the present geometric framework for the $\mathrm{E}(5 \mid 4)$ problem is obtained by replacing the bosonic operator $B^{(2)}$ with its classical limit $\alpha$. Then the matrix elements $\left\langle\tau L\|\alpha\| \tau^{\prime} L^{\prime}\right\rangle$ are evaluated using the explicit expressions for the wave functions $\Psi_{\tau \tilde{\nu}_{\Delta} L M_{L}}(\gamma, \vartheta)$ as discussed in Sec. 2.3. The resulting isoscalar factors are given in Table B.1. These isoscalar factors have phases consistent with the core reduced matrix elements of Appendix C. They are used in the calculation of the coupled system reduced matrix elements of Appendix D.

Note that the procedure for calculation of isoscalar factors described above is easily generalized to the case where degenerate $J$ values arise in the $\operatorname{Spin}(5)$ representation $\left(\tau_{1}, \frac{1}{2}\right)$. The multiplicity of $J$ is equal to the dimension of the null space of the coefficient matrix of (B.2). The null space is found by Gaussian elimination, as usual, and the isoscalar factors are obtained as the entries of an orthonormal set of basis vectors for the null space. The (arbitrary) choice of orthonormal basis determines the meaning of the multiplicity index $\widetilde{\nu}_{\triangle}^{\prime}$ for the coupled state $\left|\tau \tau_{1} \tilde{\nu}_{\triangle} J\right\rangle$. 
Table B.1

The $\operatorname{Spin}(5) \supset \operatorname{Spin}(3)$ isoscalar factors (ISFs), tabulated for $\tau_{1} \leq 7 / 2$ and omitting degenerate $J$ values (see text). A square root sign is to be understood over the magnitude of each isoscalar factor.

\begin{tabular}{rrrrrrrrrr}
\hline$\tau_{1}$ & $J$ & $\tau$ & $L$ & ISF & $\tau_{1}$ & $J$ & $\tau$ & $L$ & ISF \\
\hline $1 / 2$ & $3 / 2$ & 0 & 0 & 1 & $5 / 2$ & $11 / 2$ & 3 & 4 & $-8 / 99$ \\
$1 / 2$ & $3 / 2$ & 1 & 2 & 1 & $5 / 2$ & $11 / 2$ & 3 & 6 & $91 / 99$ \\
$3 / 2$ & $1 / 2$ & 1 & 2 & 1 & $7 / 2$ & $3 / 2$ & 3 & 0 & $2 / 3$ \\
$3 / 2$ & $5 / 2$ & 1 & 2 & 1 & $7 / 2$ & $3 / 2$ & 3 & 3 & $1 / 3$ \\
$3 / 2$ & $7 / 2$ & 1 & 2 & 1 & $7 / 2$ & $5 / 2$ & 3 & 3 & $11 / 18$ \\
$3 / 2$ & $1 / 2$ & 2 & 2 & -1 & $7 / 2$ & $5 / 2$ & 3 & 4 & $7 / 18$ \\
$3 / 2$ & $5 / 2$ & 2 & 2 & $25 / 49$ & $7 / 2$ & $7 / 2$ & 3 & 3 & $11 / 18$ \\
$3 / 2$ & $5 / 2$ & 2 & 4 & $24 / 49$ & $7 / 2$ & $7 / 2$ & 3 & 4 & $-7 / 18$ \\
$3 / 2$ & $7 / 2$ & 2 & 2 & $-4 / 49$ & $7 / 2$ & $11 / 2$ & 3 & 4 & $91 / 99$ \\
$3 / 2$ & $7 / 2$ & 2 & 4 & $45 / 49$ & $7 / 2$ & $11 / 2$ & 3 & 6 & $8 / 99$ \\
$5 / 2$ & $3 / 2$ & 2 & 2 & 1 & $7 / 2$ & $13 / 2$ & 3 & 6 & 1 \\
$5 / 2$ & $5 / 2$ & 2 & 2 & $24 / 49$ & $7 / 2$ & $15 / 2$ & 3 & 6 & 1 \\
$5 / 2$ & $5 / 2$ & 2 & 4 & $-25 / 49$ & $7 / 2$ & $3 / 2$ & 4 & 2 & 1 \\
$5 / 2$ & $7 / 2$ & 2 & 2 & $45 / 49$ & $7 / 2$ & $5 / 2$ & 4 & 2 & $12 / 77$ \\
$5 / 2$ & $7 / 2$ & 2 & 4 & $4 / 49$ & $7 / 2$ & $5 / 2$ & 4 & 4 & $-65 / 77$ \\
$5 / 2$ & $9 / 2$ & 2 & 4 & 1 & $7 / 2$ & $7 / 2$ & 4 & 2 & $-45 / 154$ \\
$5 / 2$ & $11 / 2$ & 2 & 4 & 1 & $7 / 2$ & $7 / 2$ & 4 & 4 & $-52 / 385$ \\
$5 / 2$ & $3 / 2$ & 3 & 0 & $1 / 3$ & $7 / 2$ & $7 / 2$ & 4 & 5 & $63 / 110$ \\
$5 / 2$ & $3 / 2$ & 3 & 3 & $-2 / 3$ & $7 / 2$ & $11 / 2$ & 4 & 4 & $-56 / 605$ \\
$5 / 2$ & $5 / 2$ & 3 & 3 & $-7 / 18$ & $7 / 2$ & $11 / 2$ & 4 & 5 & $9 / 55$ \\
$5 / 2$ & $5 / 2$ & 3 & 4 & $11 / 18$ & $7 / 2$ & $11 / 2$ & 4 & 6 & $90 / 121$ \\
$5 / 2$ & $7 / 2$ & 3 & 3 & $7 / 18$ & $7 / 2$ & $13 / 2$ & 4 & 5 & $-10 / 77$ \\
$5 / 2$ & $7 / 2$ & 3 & 4 & $11 / 18$ & $7 / 2$ & $13 / 2$ & 4 & 6 & $9 / 55$ \\
$5 / 2$ & $9 / 2$ & 3 & 3 & $-11 / 90$ & $7 / 2$ & $13 / 2$ & 4 & 8 & $272 / 385$ \\
$5 / 2$ & $9 / 2$ & 3 & 4 & $49 / 198$ & $7 / 2$ & $15 / 2$ & 4 & 6 & $-4 / 55$ \\
$5 / 2$ & $9 / 2$ & 3 & 6 & $104 / 165$ & $7 / 2$ & $15 / 2$ & 4 & 8 & $51 / 55$ \\
\hline & & & & & & & &
\end{tabular}




\section{Core angular reduced matrix elements}

Angular reduced matrix elements (RMEs) for the even-even core, as described in Sec. 2.3, are tabulated in Tables C.1-C.4. The matrix elements are valid for the angular solutions of $\gamma$-soft problems in general, not just for the $\mathrm{E}(5)$ case considered in this article. They also provide exact expressions for the matrix elements of transition operators in the large- $N$ limit of the IBM SO(6) dynamical symmetry. All reduced matrix elements are normalized according to the Wigner-Eckart theorem convention of Racah [54]. 
Table C.1

Angular reduced matrix elements $\left\langle L^{\prime}\|\alpha\| L\right\rangle$, between basis states related by $\Delta \tau=1$, tabulated for $\tau \leq 5$. A square root sign is to be understood over the magnitude of each matrix element.

\begin{tabular}{rrrrrrrrrr}
\hline$\tau$ & $L$ & $\tau^{\prime}$ & $L^{\prime}$ & $\mathrm{RME}$ & $\tau$ & $L$ & $\tau^{\prime}$ & $L^{\prime}$ & $\mathrm{RME}$ \\
\hline 1 & 2 & 0 & 0 & 1 & 4 & 8 & 3 & 6 & $68 / 11$ \\
2 & 2 & 1 & 2 & $-10 / 7$ & 5 & 2 & 4 & 2 & $-10 / 7$ \\
2 & 4 & 1 & 2 & $18 / 7$ & 5 & 2 & 4 & 4 & $45 / 91$ \\
3 & 0 & 2 & 2 & $1 / 3$ & 5 & 4 & 4 & 2 & $135 / 77$ \\
3 & 3 & 2 & 2 & $5 / 3$ & 5 & 4 & 4 & 4 & $15552 / 55055$ \\
3 & 3 & 2 & 4 & $2 / 3$ & 5 & 4 & 4 & 5 & $63 / 55$ \\
3 & 4 & 2 & 2 & $11 / 7$ & 5 & 4 & 4 & 6 & $441 / 1573$ \\
3 & 4 & 2 & 4 & $-10 / 7$ & 5 & 5 & 4 & 4 & $189 / 65$ \\
3 & 6 & 2 & 4 & $13 / 3$ & 5 & 5 & 4 & 5 & $-66 / 65$ \\
4 & 2 & 3 & 0 & $2 / 3$ & 5 & 5 & 4 & 6 & $4 / 13$ \\
4 & 2 & 3 & 3 & $5 / 6$ & 5 & 6 & 4 & 4 & $3213 / 1573$ \\
4 & 2 & 3 & 4 & $7 / 22$ & 5 & 6 & 4 & 5 & $68 / 77$ \\
4 & 4 & 3 & 3 & $26 / 15$ & 5 & 6 & 4 & 6 & $-1224 / 605$ \\
4 & 4 & 3 & 4 & $-182 / 121$ & 5 & 6 & 4 & 8 & $-256 / 5005$ \\
4 & 4 & 3 & 6 & $-64 / 1815$ & 5 & 7 & 4 & 5 & $360 / 91$ \\
4 & 5 & 3 & 3 & $21 / 10$ & 5 & 7 & 4 & 6 & $9 / 13$ \\
4 & 5 & 3 & 4 & $21 / 22$ & 5 & 7 & 4 & 8 & $102 / 91$ \\
4 & 5 & 3 & 6 & $52 / 55$ & 5 & 8 & 4 & 6 & $323 / 65$ \\
4 & 6 & 3 & 4 & $390 / 121$ & 5 & 8 & 4 & 8 & $-102 / 65$ \\
4 & 6 & 3 & 6 & $-182 / 121$ & 5 & 10 & 4 & 8 & $105 / 13$ \\
\hline
\end{tabular}


Table C.2

Angular reduced matrix elements $\left\langle L^{\prime}\left\|(\alpha \times \alpha)^{(2)}\right\| L\right\rangle$, between basis states related by $\Delta \tau=0$, tabulated for $\tau \leq 5$. A square root sign is to be understood over the magnitude of each matrix element.

\begin{tabular}{rrrrrrrrrr}
\hline$\tau$ & $L$ & $\tau^{\prime}$ & $L^{\prime}$ & $\mathrm{RME}$ & $\tau$ & $L$ & $\tau^{\prime}$ & $L^{\prime}$ & $\mathrm{RME}$ \\
\hline 1 & 2 & 1 & 2 & $20 / 49$ & 4 & 8 & 4 & 8 & $15504 / 5915$ \\
2 & 2 & 2 & 2 & $-20 / 441$ & 5 & 2 & 5 & 2 & $-4 / 245$ \\
2 & 2 & 2 & 4 & $-64 / 441$ & 5 & 2 & 5 & 4 & $-96 / 2695$ \\
2 & 4 & 2 & 2 & $-64 / 441$ & 5 & 4 & 5 & 2 & $-96 / 2695$ \\
2 & 4 & 2 & 4 & $440 / 441$ & 5 & 4 & 5 & 4 & $1527752 / 8152375$ \\
3 & 3 & 3 & 3 & 0 & 5 & 4 & 5 & 5 & $32 / 1375$ \\
3 & 3 & 3 & 4 & $-24 / 77$ & 5 & 4 & 5 & 6 & $2176 / 33275$ \\
3 & 4 & 3 & 3 & 5 & 5 & 5 & 4 & $-32 / 1375$ \\
3 & 4 & 3 & 4 & $5760 / 65219$ & 5 & 5 & 5 & 5 & $-1188 / 11375$ \\
3 & 4 & 3 & 6 & $-2496 / 9317$ & 5 & 5 & 5 & 6 & $4896 / 13475$ \\
3 & 6 & 3 & 4 & $-2496 / 9317$ & 5 & 5 & 5 & 7 & $-256 / 3185$ \\
3 & 6 & 3 & 6 & $2340 / 1331$ & 5 & 6 & 5 & 4 & $2176 / 33275$ \\
4 & 2 & 4 & 2 & $20 / 441$ & 5 & 6 & 5 & 5 & $-4896 / 13475$ \\
4 & 2 & 4 & 4 & $160 / 5733$ & 5 & 6 & 5 & 6 & $247572 / 8152375$ \\
4 & 4 & 4 & 2 & $160 / 5733$ & 5 & 6 & 5 & 7 & $2176 / 13475$ \\
4 & 4 & 4 & 4 & $-356168 / 4099095$ & 5 & 6 & 5 & 8 & $-21888 / 67375$ \\
4 & 4 & 4 & 5 & $32 / 195$ & 5 & 7 & 5 & 5 & $-256 / 3185$ \\
4 & 4 & 4 & 6 & $-896 / 5577$ & 5 & 7 & 5 & 6 & $-2176 / 13475$ \\
4 & 5 & 4 & 4 & $-32 / 195$ & 5 & 7 & 5 & 7 & $8228 / 9555$ \\
4 & 5 & 4 & 5 & $132 / 455$ & 5 & 7 & 5 & 8 & $1292 / 3675$ \\
4 & 5 & 4 & 6 & $32 / 91$ & 5 & 8 & 5 & 6 & $-21888 / 67375$ \\
4 & 6 & 4 & 4 & $-896 / 5577$ & 5 & 8 & 5 & 7 & $-1292 / 3675$ \\
4 & 6 & 4 & 5 & $-32 / 91$ & 5 & 8 & 5 & 8 & $16524 / 16625$ \\
4 & 6 & 4 & 6 & $324 / 715$ & 5 & 8 & 5 & 10 & $-128 / 285$ \\
4 & 6 & 4 & 8 & $-2176 / 5915$ & 5 & 10 & 5 & 8 & $-128 / 285$ \\
4 & 8 & 4 & 6 & $-2176 / 5915$ & 5 & 10 & 5 & 10 & $1012 / 285$ \\
\hline & & & & & & &
\end{tabular}


Table C.3

Angular reduced matrix elements $\left\langle L^{\prime}\left\|(\alpha \times \alpha)^{(2)}\right\| L\right\rangle$, between basis states related by $\Delta \tau=2$, tabulated for $\tau \leq 5$. A square root sign is to be understood over the magnitude of each matrix element.

\begin{tabular}{rrrrrrrrrr}
\hline$\tau$ & $L$ & $\tau^{\prime}$ & $L^{\prime}$ & $\mathrm{RME}$ & $\tau$ & $L$ & $\tau^{\prime}$ & $L^{\prime}$ & $\mathrm{RME}$ \\
\hline 2 & 2 & 0 & 0 & $-2 / 7$ & 5 & 2 & 3 & 4 & $-1 / 11$ \\
3 & 0 & 1 & 2 & $-2 / 21$ & 5 & 4 & 3 & 3 & $144 / 385$ \\
3 & 3 & 1 & 2 & $10 / 21$ & 5 & 4 & 3 & 4 & $432 / 1331$ \\
3 & 4 & 1 & 2 & $-22 / 49$ & 5 & 4 & 3 & 6 & $-66978 / 605605$ \\
4 & 2 & 2 & 2 & $160 / 441$ & 5 & 5 & 3 & 3 & $-3 / 5$ \\
4 & 2 & 2 & 4 & $-50 / 441$ & 5 & 5 & 3 & 4 & $3 / 11$ \\
4 & 4 & 2 & 2 & $-260 / 441$ & 5 & 5 & 3 & 6 & $32 / 5005$ \\
4 & 4 & 2 & 4 & $-416 / 4851$ & 5 & 6 & 3 & 4 & $-1020 / 1331$ \\
4 & 5 & 2 & 4 & $2 / 3$ & 5 & 6 & 3 & 6 & $-1088 / 9317$ \\
4 & 6 & 2 & 4 & $-130 / 231$ & 5 & 7 & 3 & 6 & $810 / 1001$ \\
5 & 2 & 3 & 0 & $-4 / 21$ & 5 & 8 & 3 & 6 & $-646 / 1001$ \\
5 & 2 & 3 & 3 & $5 / 21$ & & & & & \\
\hline
\end{tabular}

Table C.4

Angular reduced matrix elements $\left\langle L^{\prime}\left\|\left[\alpha \times i(\alpha \times \tilde{\pi})^{(1)}\right]^{(1)}\right\| L\right\rangle$, between basis states related by $\Delta \tau=1$, tabulated for $\tau \leq 5$. A square root sign is to be understood over the magnitude of each matrix element.

\begin{tabular}{rrrrrrrrrr}
\hline$\tau$ & $L$ & $\tau^{\prime}$ & $L^{\prime}$ & $\mathrm{RME}$ & $\tau$ & $L$ & $\tau^{\prime}$ & $L^{\prime}$ & $\mathrm{RME}$ \\
\hline 2 & 2 & 1 & 2 & $3 / 10$ & 5 & 4 & 4 & 4 & $-3888 / 17875$ \\
3 & 3 & 2 & 2 & $-2 / 5$ & 5 & 4 & 4 & 5 & $-1134 / 1375$ \\
3 & 3 & 2 & 4 & $-3 / 10$ & 5 & 5 & 4 & 4 & $-3402 / 1625$ \\
3 & 4 & 2 & 4 & $11 / 10$ & 5 & 5 & 4 & 5 & $297 / 250$ \\
4 & 2 & 3 & 3 & $-1 / 5$ & 5 & 5 & 4 & 6 & $-21 / 65$ \\
4 & 4 & 3 & 3 & $-39 / 50$ & 5 & 6 & 4 & 5 & $-51 / 55$ \\
4 & 4 & 3 & 4 & $637 / 550$ & 5 & 6 & 4 & 6 & $918 / 275$ \\
4 & 5 & 3 & 4 & $-189 / 275$ & 5 & 7 & 4 & 6 & $-324 / 325$ \\
4 & 5 & 3 & 6 & $-273 / 275$ & 5 & 7 & 4 & 8 & $-1377 / 650$ \\
4 & 6 & 3 & 6 & $273 / 110$ & 5 & 8 & 4 & 8 & $2907 / 650$ \\
5 & 2 & 4 & 2 & $3 / 10$ & & & & & \\
\hline
\end{tabular}




\section{Coupled system reduced matrix elements}

Angular reduced matrix elements (RMEs) for the coupled odd-mass system, as described in Sec. 3.2, are taulated in Tables D.1-D.6. The matrix elements are applicable to problems involving a $\gamma$-soft core coupled to a $j=3 / 2$ particle in general, not just to the $\mathrm{E}(5 \mid 4)$ case considered in this article. All reduced matrix elements are normalized according to the Wigner-Eckart theorem convention of Racah [54].

In Tables D.1 and D.2, certain redundant matrix element values have been suppressed for conciseness. In Table D.1, only the $\Delta \tau_{1}=1$ matrix elements within the $\tau_{1}=\tau+1 / 2$ family of states are tabulated, since those within the $\tau_{1}=\tau-1 / 2$ family are identical. In Table D.2, only the $\Delta \tau_{1}=0$ or 2 matrix elements within the $\tau_{1}=\tau+1 / 2$ family of states are tabulated, since those within the $\tau_{1}=\tau-1 / 2$ family are identical. Also, only the $\Delta \tau_{1}=1$ matrix elements with $\Delta \tau=0$ are tabulated, since those with $\Delta \tau=2$ are identical. 
Table D.1

Angular reduced matrix elements $\left\langle J^{\prime}\|\alpha\| J\right\rangle$ between Spin(5) coupled states, for $\tau_{1} \leq$ $5 / 2$. A square root sign is to be understood over the magnitude of each matrix element.

\begin{tabular}{|c|c|c|c|c|c|c|c|c|c|c|c|c|c|}
\hline$\tau$ & $\tau_{1}$ & $J$ & $\overrightarrow{\tau^{\prime}}$ & $\tau_{1}^{\prime}$ & $J^{\prime}$ & $\overline{\mathrm{RME}}$ & $\tau$ & $\tau_{1}$ & $J$ & $\tau^{\prime}$ & $\tau_{1}^{\prime}$ & $J^{\prime}$ & RME \\
\hline 1 & $1 / 2$ & $3 / 2$ & 0 & $1 / 2$ & $3 / 2$ & $4 / 5$ & 3 & $5 / 2$ & $9 / 2$ & 2 & $5 / 2$ & $7 / 2$ & $32 / 11907$ \\
\hline 2 & $3 / 2$ & $1 / 2$ & 1 & $3 / 2$ & $5 / 2$ & $6 / 35$ & 3 & $5 / 2$ & $9 / 2$ & 2 & $5 / 2$ & $9 / 2$ & $-125 / 2673$ \\
\hline 2 & $3 / 2$ & $5 / 2$ & 1 & $3 / 2$ & $1 / 2$ & $6 / 35$ & 3 & $5 / 2$ & $9 / 2$ & 2 & $5 / 2$ & $11 / 2$ & $-832 / 2673$ \\
\hline 2 & $3 / 2$ & $5 / 2$ & 1 & $3 / 2$ & $5 / 2$ & $-12 / 1715$ & 3 & $5 / 2$ & $11 / 2$ & 2 & $5 / 2$ & $7 / 2$ & $128 / 8505$ \\
\hline 2 & $3 / 2$ & $5 / 2$ & 1 & $3 / 2$ & $7 / 2$ & $-576 / 1715$ & 3 & $5 / 2$ & $11 / 2$ & 2 & $5 / 2$ & $9 / 2$ & $832 / 2673$ \\
\hline 2 & $3 / 2$ & $7 / 2$ & 1 & $3 / 2$ & $5 / 2$ & $576 / 1715$ & 3 & $5 / 2$ & $11 / 2$ & 2 & $5 / 2$ & $11 / 2$ & $364 / 1485$ \\
\hline 2 & $3 / 2$ & $7 / 2$ & 1 & $3 / 2$ & $7 / 2$ & $120 / 343$ & 2 & $3 / 2$ & $1 / 2$ & 1 & $1 / 2$ & $3 / 2$ & $2 / 5$ \\
\hline 3 & $5 / 2$ & $3 / 2$ & 2 & $5 / 2$ & $3 / 2$ & $-4 / 45$ & 2 & $3 / 2$ & $5 / 2$ & 1 & $1 / 2$ & $3 / 2$ & $6 / 5$ \\
\hline 3 & $5 / 2$ & $3 / 2$ & 2 & $5 / 2$ & $5 / 2$ & $-64 / 2205$ & 2 & $3 / 2$ & $7 / 2$ & 1 & $1 / 2$ & $3 / 2$ & $8 / 5$ \\
\hline 3 & $5 / 2$ & $3 / 2$ & 2 & $5 / 2$ & $7 / 2$ & $32 / 441$ & 3 & $5 / 2$ & $3 / 2$ & 2 & $3 / 2$ & $1 / 2$ & $2 / 5$ \\
\hline 3 & $5 / 2$ & $5 / 2$ & 2 & $5 / 2$ & $3 / 2$ & $64 / 2205$ & 3 & $5 / 2$ & $3 / 2$ & 2 & $3 / 2$ & $5 / 2$ & $-30 / 49$ \\
\hline 3 & $5 / 2$ & $5 / 2$ & 2 & $5 / 2$ & $5 / 2$ & $121 / 46305$ & 3 & $5 / 2$ & $3 / 2$ & 2 & $3 / 2$ & $7 / 2$ & $-32 / 245$ \\
\hline 3 & $5 / 2$ & $5 / 2$ & 2 & $5 / 2$ & $7 / 2$ & $-11552 / 83349$ & 3 & $5 / 2$ & $5 / 2$ & 2 & $3 / 2$ & $1 / 2$ & $-36 / 35$ \\
\hline 3 & $5 / 2$ & $5 / 2$ & 2 & $5 / 2$ & $9 / 2$ & $-1375 / 11907$ & 3 & $5 / 2$ & $5 / 2$ & 2 & $3 / 2$ & $5 / 2$ & $-90 / 343$ \\
\hline 3 & $5 / 2$ & $7 / 2$ & 2 & $5 / 2$ & $3 / 2$ & $32 / 441$ & 3 & $5 / 2$ & $5 / 2$ & 2 & $3 / 2$ & $7 / 2$ & $-726 / 1715$ \\
\hline 3 & $5 / 2$ & $7 / 2$ & 2 & $5 / 2$ & $5 / 2$ & $11552 / 83349$ & 3 & $5 / 2$ & $7 / 2$ & 2 & $3 / 2$ & $5 / 2$ & $400 / 343$ \\
\hline 3 & $5 / 2$ & $7 / 2$ & 2 & $5 / 2$ & $7 / 2$ & $63368 / 416745$ & 3 & $5 / 2$ & $7 / 2$ & 2 & $3 / 2$ & $7 / 2$ & $-384 / 343$ \\
\hline 3 & $5 / 2$ & $7 / 2$ & 2 & $5 / 2$ & $9 / 2$ & $-32 / 11907$ & 3 & $5 / 2$ & $9 / 2$ & 2 & $3 / 2$ & $5 / 2$ & $110 / 49$ \\
\hline 3 & $5 / 2$ & $7 / 2$ & 2 & $5 / 2$ & $11 / 2$ & $128 / 8505$ & 3 & $5 / 2$ & $9 / 2$ & 2 & $3 / 2$ & $7 / 2$ & $30 / 49$ \\
\hline 3 & $5 / 2$ & $9 / 2$ & 2 & $5 / 2$ & $5 / 2$ & $-1375 / 11907$ & 3 & $5 / 2$ & $11 / 2$ & 2 & $3 / 2$ & $7 / 2$ & $24 / 7$ \\
\hline
\end{tabular}

Table D.2

Angular reduced matrix elements $\left\langle J^{\prime}\left\|(\alpha \times \alpha)^{(2)}\right\| J\right\rangle$ between Spin(5) coupled states, for $\tau_{1} \leq 5 / 2$. A square root sign is to be understood over the magnitude of each matrix element.

\begin{tabular}{|c|c|c|c|c|c|c|c|c|c|c|c|c|c|}
\hline$\tau$ & $\tau_{1}$ & $J$ & $\tau^{\prime}$ & $\tau_{1}^{\prime}$ & $J^{\prime}$ & RME & $\tau$ & $\tau_{1}$ & $J$ & $\bar{\tau}^{\prime}$ & $\tau_{1}^{\prime}$ & $J^{\prime}$ & RME \\
\hline 1 & $1 / 2$ & $3 / 2$ & 1 & $1 / 2$ & $3 / 2$ & $\overline{0}$ & 3 & $5 / 2$ & $9 / 2$ & $\overline{3}$ & $5 / 2$ & $11 / 2$ & $416 / 2079$ \\
\hline 2 & $3 / 2$ & $1 / 2$ & 2 & $3 / 2$ & $5 / 2$ & $12 / 245$ & 3 & $5 / 2$ & $11 / 2$ & 3 & $5 / 2$ & $7 / 2$ & $-1024 / 6615$ \\
\hline 2 & $3 / 2$ & $5 / 2$ & 2 & $3 / 2$ & $1 / 2$ & $12 / 245$ & 3 & $5 / 2$ & $11 / 2$ & 3 & $5 / 2$ & $9 / 2$ & $-416 / 2079$ \\
\hline 2 & $3 / 2$ & $5 / 2$ & 2 & $3 / 2$ & $5 / 2$ & $120 / 2401$ & 3 & $5 / 2$ & $11 / 2$ & 3 & $5 / 2$ & $11 / 2$ & $1664 / 1485$ \\
\hline 2 & $3 / 2$ & $5 / 2$ & 2 & $3 / 2$ & $7 / 2$ & $2592 / 12005$ & 1 & $3 / 2$ & $1 / 2$ & 1 & $1 / 2$ & $3 / 2$ & $4 / 35$ \\
\hline 2 & $3 / 2$ & $7 / 2$ & 2 & $3 / 2$ & $5 / 2$ & $-2592 / 12005$ & 1 & $3 / 2$ & $5 / 2$ & 1 & $1 / 2$ & $3 / 2$ & $-60 / 343$ \\
\hline 2 & $3 / 2$ & $7 / 2$ & 2 & $3 / 2$ & $7 / 2$ & $960 / 2401$ & 1 & $3 / 2$ & $7 / 2$ & 1 & $1 / 2$ & $3 / 2$ & $64 / 1715$ \\
\hline 3 & $5 / 2$ & $3 / 2$ & 3 & $5 / 2$ & $3 / 2$ & 0 & 2 & $5 / 2$ & $3 / 2$ & 2 & $3 / 2$ & $1 / 2$ & $-4 / 315$ \\
\hline 3 & $5 / 2$ & $3 / 2$ & 3 & $5 / 2$ & $5 / 2$ & $-160 / 3087$ & 2 & $5 / 2$ & $3 / 2$ & 2 & $3 / 2$ & $5 / 2$ & $4 / 5145$ \\
\hline 3 & $5 / 2$ & $3 / 2$ & 3 & $5 / 2$ & $7 / 2$ & $-64 / 3087$ & 2 & $5 / 2$ & $3 / 2$ & 2 & $3 / 2$ & $7 / 2$ & $-1024 / 15435$ \\
\hline 3 & $5 / 2$ & $5 / 2$ & 3 & $5 / 2$ & $3 / 2$ & $160 / 3087$ & 2 & $5 / 2$ & $5 / 2$ & 2 & $3 / 2$ & $1 / 2$ & $-32 / 2205$ \\
\hline 3 & $5 / 2$ & $5 / 2$ & 3 & $5 / 2$ & $5 / 2$ & $9610 / 64827$ & 2 & $5 / 2$ & $5 / 2$ & 2 & $3 / 2$ & $5 / 2$ & $-15376 / 108045$ \\
\hline 3 & $5 / 2$ & $5 / 2$ & 3 & $5 / 2$ & $7 / 2$ & $-48 / 2401$ & 2 & $5 / 2$ & $5 / 2$ & 2 & $3 / 2$ & $7 / 2$ & $-5476 / 324135$ \\
\hline 3 & $5 / 2$ & $5 / 2$ & 3 & $5 / 2$ & $9 / 2$ & $-110 / 1029$ & 2 & $5 / 2$ & $7 / 2$ & 2 & $3 / 2$ & $5 / 2$ & $-32 / 194481$ \\
\hline 3 & $5 / 2$ & $7 / 2$ & 3 & $5 / 2$ & $3 / 2$ & $-64 / 3087$ & 2 & $5 / 2$ & $7 / 2$ & 2 & $3 / 2$ & $7 / 2$ & $256 / 7203$ \\
\hline 3 & $5 / 2$ & $7 / 2$ & 3 & $5 / 2$ & $5 / 2$ & $48 / 2401$ & 2 & $5 / 2$ & $9 / 2$ & 2 & $3 / 2$ & $5 / 2$ & $-880 / 27783$ \\
\hline 3 & $5 / 2$ & $7 / 2$ & 3 & $5 / 2$ & $7 / 2$ & $-87616 / 2917215$ & 2 & $5 / 2$ & $9 / 2$ & 2 & $3 / 2$ & $7 / 2$ & $-60 / 343$ \\
\hline 3 & $5 / 2$ & $7 / 2$ & 3 & $5 / 2$ & $9 / 2$ & $8464 / 83349$ & 2 & $5 / 2$ & $11 / 2$ & 2 & $3 / 2$ & $7 / 2$ & $64 / 1323$ \\
\hline 3 & $5 / 2$ & $7 / 2$ & 3 & $5 / 2$ & $11 / 2$ & $-1024 / 6615$ & 3 & $5 / 2$ & $3 / 2$ & 1 & $1 / 2$ & $3 / 2$ & $-8 / 35$ \\
\hline 3 & $5 / 2$ & $9 / 2$ & 3 & $5 / 2$ & $5 / 2$ & $-110 / 1029$ & 3 & $5 / 2$ & $5 / 2$ & 1 & $1 / 2$ & $3 / 2$ & $-288 / 1715$ \\
\hline 3 & $5 / 2$ & $9 / 2$ & 3 & $5 / 2$ & $7 / 2$ & $-8464 / 83349$ & 3 & $5 / 2$ & $7 / 2$ & 1 & $1 / 2$ & $3 / 2$ & $-144 / 343$ \\
\hline 3 & $5 / 2$ & $9 / 2$ & 3 & $5 / 2$ & $9 / 2$ & $1750 / 2673$ & & & & & & & \\
\hline
\end{tabular}


Table D.3

Angular reduced matrix elements $\left\langle J^{\prime}\left\|\left(a^{\dagger} \times \tilde{a}\right)^{(2)}\right\| J\right\rangle$ between Spin(5) coupled states, for $\tau_{1} \leq 5 / 2$. A square root sign is to be understood over the magnitude of each matrix element.

\begin{tabular}{|c|c|c|c|c|c|c|c|c|c|c|c|c|c|}
\hline$\tau$ & $\tau_{1}$ & $J$ & $\tau^{\prime}$ & $\tau_{1}^{\prime}$ & $J^{\prime}$ & RME & $\tau$ & $\tau_{1}$ & $J$ & $\tau^{\prime}$ & $\tau_{1}^{\prime}$ & $J^{\prime}$ & $\overline{\mathrm{RME}}$ \\
\hline 0 & $1 / 2$ & $3 / 2$ & 0 & $1 / 2$ & $3 / 2$ & -5 & 3 & $5 / 2$ & $3 / 2$ & 3 & $5 / \frac{1}{2}$ & $3 / 2$ & $-49 / 45$ \\
\hline 1 & $3 / 2$ & $1 / 2$ & 1 & $3 / 2$ & $5 / 2$ & $-21 / 10$ & 3 & $5 / 2$ & $3 / 2$ & 3 & $5 / 2$ & $5 / 2$ & $-16 / 45$ \\
\hline 1 & $3 / 2$ & $5 / 2$ & 1 & $3 / 2$ & $1 / 2$ & $-21 / 10$ & 3 & $5 / 2$ & $3 / 2$ & 3 & $5 / 2$ & $7 / 2$ & $8 / 9$ \\
\hline 1 & $3 / 2$ & $5 / 2$ & 1 & $3 / 2$ & $5 / 2$ & $3 / 35$ & 3 & $5 / 2$ & $5 / 2$ & 3 & $5 / 2$ & $3 / 2$ & $16 / 45$ \\
\hline 1 & $3 / 2$ & $5 / 2$ & 1 & $3 / 2$ & $7 / 2$ & $144 / 35$ & 3 & $5 / 2$ & $5 / 2$ & 3 & $5 / 2$ & $5 / 2$ & $121 / 3780$ \\
\hline 1 & $3 / 2$ & $7 / 2$ & 1 & $3 / 2$ & $5 / 2$ & $-144 / 35$ & 3 & $5 / 2$ & $5 / 2$ & 3 & $5 / 2$ & $7 / 2$ & $-2888 / 1701$ \\
\hline 1 & $3 / 2$ & $7 / 2$ & 1 & $3 / 2$ & $7 / 2$ & $-30 / 7$ & 3 & $5 / 2$ & $5 / 2$ & 3 & $5 / 2$ & $9 / 2$ & $-1375 / 972$ \\
\hline 2 & $5 / 2$ & $3 / 2$ & 2 & $5 / 2$ & $3 / 2$ & $9 / 5$ & 3 & $5 / 2$ & $7 / 2$ & 3 & $5 / 2$ & $3 / 2$ & $8 / 9$ \\
\hline 2 & $5 / 2$ & $3 / 2$ & 2 & $5 / 2$ & $5 / 2$ & $144 / 245$ & 3 & $5 / 2$ & $7 / 2$ & 3 & $5 / 2$ & $5 / 2$ & $2888 / 1701$ \\
\hline 2 & $5 / 2$ & $3 / 2$ & 2 & $5 / 2$ & $7 / 2$ & $-72 / 49$ & 3 & $5 / 2$ & $7 / 2$ & 3 & $5 / 2$ & $7 / 2$ & $15842 / 8505$ \\
\hline 2 & $5 / 2$ & $5 / 2$ & 2 & $5 / 2$ & $3 / 2$ & $-144 / 245$ & 3 & $5 / 2$ & $7 / 2$ & 3 & $5 / 2$ & $9 / 2$ & $-8 / 243$ \\
\hline 2 & $5 / 2$ & $5 / 2$ & 2 & $5 / 2$ & $5 / 2$ & $-363 / 6860$ & 3 & $5 / 2$ & $7 / 2$ & 3 & $5 / 2$ & $11 / 2$ & $224 / 1215$ \\
\hline 2 & $5 / 2$ & $5 / 2$ & 2 & $5 / 2$ & $7 / 2$ & $2888 / 1029$ & 3 & $5 / 2$ & $9 / 2$ & 3 & $5 / 2$ & $5 / 2$ & $-1375 / 972$ \\
\hline 2 & $5 / 2$ & $5 / 2$ & 2 & $5 / 2$ & $9 / 2$ & $1375 / 588$ & 3 & $5 / 2$ & $9 / 2$ & 3 & $5 / 2$ & $7 / 2$ & $8 / 243$ \\
\hline 2 & $5 / 2$ & $7 / 2$ & 2 & $5 / 2$ & $3 / 2$ & $-72 / 49$ & 3 & $5 / 2$ & $9 / 2$ & 3 & $5 / 2$ & $9 / 2$ & $-6125 / 10692$ \\
\hline 2 & $5 / 2$ & $7 / 2$ & 2 & $5 / 2$ & $5 / 2$ & $-2888 / 1029$ & 3 & $5 / 2$ & $9 / 2$ & 3 & $5 / 2$ & $11 / 2$ & $-10192 / 2673$ \\
\hline 2 & $5 / 2$ & $7 / 2$ & 2 & $5 / 2$ & $7 / 2$ & $-15842 / 5145$ & 3 & $5 / 2$ & $11 / 2$ & 3 & $5 / 2$ & $7 / 2$ & $224 / 1215$ \\
\hline 2 & $5 / 2$ & $7 / 2$ & 2 & $5 / 2$ & $9 / 2$ & $8 / 147$ & 3 & $5 / 2$ & $11 / 2$ & 3 & $5 / 2$ & $9 / 2$ & $10192 / 2673$ \\
\hline 2 & $5 / 2$ & $7 / 2$ & 2 & $5 / 2$ & $11 / 2$ & $-32 / 105$ & 3 & $5 / 2$ & $11 / 2$ & 3 & $5 / 2$ & $11 / 2$ & $4459 / 1485$ \\
\hline 2 & $5 / 2$ & $9 / 2$ & 2 & $5 / 2$ & $5 / 2$ & $1375 / 588$ & 1 & $3 / 2$ & $1 / 2$ & 1 & $1 / 2$ & $3 / 2$ & $-2 / 5$ \\
\hline 2 & $5 / 2$ & $9 / 2$ & 2 & $5 / 2$ & $7 / 2$ & $-8 / 147$ & 1 & $3 / 2$ & $5 / 2$ & 1 & $1 / 2$ & $3 / 2$ & $-6 / 5$ \\
\hline 2 & $5 / 2$ & $9 / 2$ & 2 & $5 / 2$ & $9 / 2$ & $125 / 132$ & 1 & $3 / 2$ & $7 / 2$ & 1 & $1 / 2$ & $3 / 2$ & $-8 / 5$ \\
\hline 2 & $5 / 2$ & $9 / 2$ & 2 & $5 / 2$ & $11 / 2$ & $208 / 33$ & 2 & $5 / 2$ & $3 / 2$ & 2 & $3 / 2$ & $1 / 2$ & $-2 / 5$ \\
\hline 2 & $5 / 2$ & $11 / 2$ & 2 & $5 / 2$ & $7 / 2$ & $-32 / 105$ & 2 & $5 / 2$ & $3 / 2$ & 2 & $3 / 2$ & $5 / 2$ & $30 / 49$ \\
\hline 2 & $5 / 2$ & $11 / 2$ & 2 & $5 / 2$ & $9 / 2$ & $-208 / 33$ & 2 & $5 / 2$ & $3 / 2$ & 2 & $3 / 2$ & $7 / 2$ & $32 / 245$ \\
\hline 2 & $5 / 2$ & $11 / 2$ & 2 & $5 / 2$ & $11 / 2$ & $-273 / 55$ & 2 & $5 / 2$ & $5 / 2$ & 2 & $3 / 2$ & $1 / 2$ & $36 / 35$ \\
\hline 1 & $1 / 2$ & $3 / 2$ & 1 & $1 / 2$ & $3 / 2$ & $9 / 5$ & 2 & $5 / 2$ & $5 / 2$ & 2 & $3 / 2$ & $5 / 2$ & $90 / 343$ \\
\hline 2 & $3 / 2$ & $1 / 2$ & 2 & $3 / 2$ & $5 / 2$ & $15 / 14$ & 2 & $5 / 2$ & $5 / 2$ & 2 & $3 / 2$ & $7 / 2$ & $726 / 1715$ \\
\hline 2 & $3 / 2$ & $5 / 2$ & 2 & $3 / 2$ & $1 / 2$ & $15 / 14$ & 2 & $5 / 2$ & $7 / 2$ & 2 & $3 / 2$ & $5 / 2$ & $-400 / 343$ \\
\hline 2 & $3 / 2$ & $5 / 2$ & 2 & $3 / 2$ & $5 / 2$ & $-15 / 343$ & 2 & $5 / 2$ & $7 / 2$ & 2 & $3 / 2$ & $7 / 2$ & $384 / 343$ \\
\hline 2 & $3 / 2$ & $5 / 2$ & 2 & $3 / 2$ & $7 / 2$ & $-720 / 343$ & 2 & $5 / 2$ & $9 / 2$ & 2 & $3 / 2$ & $5 / 2$ & $-110 / 49$ \\
\hline 2 & $3 / 2$ & $7 / 2$ & 2 & $3 / 2$ & $5 / 2$ & $720 / 343$ & 2 & $5 / 2$ & $9 / 2$ & 2 & $3 / 2$ & $7 / 2$ & $-30 / 49$ \\
\hline 2 & $3 / 2$ & $7 / 2$ & 2 & $3 / 2$ & $7 / 2$ & $750 / 343$ & 2 & $5 / 2$ & $11 / 2$ & 2 & $3 / 2$ & $7 / 2$ & $-24 / 7$ \\
\hline
\end{tabular}

Table D.4

Angular reduced matrix elements $\left\langle J^{\prime}\left\|i(\alpha \times \tilde{\pi})^{(1)}\right\| J\right\rangle$ between Spin(5) coupled states, for $\tau_{1} \leq 5 / 2$. For reduced matrix elements of $\hat{L}$, multiply by $\sqrt{10}$. A square root sign is to be understood over the magnitude of each matrix element.

\begin{tabular}{|c|c|c|c|c|c|c|c|c|c|c|c|c|c|}
\hline$\tau$ & $\tau_{1}$ & $J$ & $\tau^{\prime}$ & $\tau_{1}^{\prime}$ & $J^{\prime}$ & RME & $\tau$ & $\tau_{1}$ & $J$ & $\tau^{\prime}$ & $\tau_{1}^{\prime}$ & $J^{\prime}$ & RME \\
\hline 0 & $1 / 2$ & $3 / 2$ & 0 & $1 / 2$ & $3 / 2$ & 0 & 2 & $3 / 2$ & $7 / 2$ & 2 & $3 / 2$ & $7 / 2$ & $20736 / 1715$ \\
\hline 1 & $3 / 2$ & $1 / 2$ & 1 & $3 / 2$ & $1 / 2$ & $3 / 5$ & 3 & $5 / 2$ & $3 / 2$ & 3 & $5 / 2$ & $3 / 2$ & $128 / 75$ \\
\hline 1 & $3 / 2$ & $5 / 2$ & 1 & $3 / 2$ & $5 / 2$ & $363 / 175$ & 3 & $5 / 2$ & $3 / 2$ & 3 & $5 / 2$ & $5 / 2$ & $56 / 225$ \\
\hline 1 & $3 / 2$ & $5 / 2$ & 1 & $3 / 2$ & $7 / 2$ & $24 / 35$ & 3 & $5 / 2$ & $5 / 2$ & 3 & $5 / 2$ & $3 / 2$ & $-56 / 225$ \\
\hline 1 & $3 / 2$ & $7 / 2$ & 1 & $3 / 2$ & $5 / 2$ & $-24 / 35$ & 3 & $5 / 2$ & $5 / 2$ & 3 & $5 / 2$ & $5 / 2$ & $38809 / 4725$ \\
\hline 1 & $3 / 2$ & $7 / 2$ & 1 & $3 / 2$ & $7 / 2$ & $144 / 35$ & 3 & $5 / 2$ & $5 / 2$ & 3 & $5 / 2$ & $7 / 2$ & $4 / 63$ \\
\hline 2 & $5 / 2$ & $3 / 2$ & 2 & $5 / 2$ & $3 / 2$ & $24 / 25$ & 3 & $5 / 2$ & $7 / 2$ & 3 & $5 / 2$ & $5 / 2$ & $-4 / 63$ \\
\hline 2 & $5 / 2$ & $3 / 2$ & 2 & $5 / 2$ & $5 / 2$ & $72 / 175$ & 3 & $5 / 2$ & $7 / 2$ & 3 & $5 / 2$ & $7 / 2$ & $54080 / 5103$ \\
\hline 2 & $5 / 2$ & $5 / 2$ & 2 & $5 / 2$ & $3 / 2$ & $-72 / 175$ & 3 & $5 / 2$ & $7 / 2$ & 3 & $5 / 2$ & $9 / 2$ & $308 / 3645$ \\
\hline 2 & $5 / 2$ & $5 / 2$ & 2 & $5 / 2$ & $5 / 2$ & $48387 / 8575$ & 3 & $5 / 2$ & $9 / 2$ & 3 & $5 / 2$ & $7 / 2$ & $-308 / 3645$ \\
\hline 2 & $5 / 2$ & $5 / 2$ & 2 & $5 / 2$ & $7 / 2$ & $36 / 343$ & 3 & $5 / 2$ & $9 / 2$ & 3 & $5 / 2$ & $9 / 2$ & $235225 / 8019$ \\
\hline 2 & $5 / 2$ & $7 / 2$ & 2 & $5 / 2$ & $5 / 2$ & $-36 / 343$ & 3 & $5 / 2$ & $9 / 2$ & 3 & $5 / 2$ & $11 / 2$ & $392 / 495$ \\
\hline 2 & $5 / 2$ & $7 / 2$ & 2 & $5 / 2$ & $7 / 2$ & $71824 / 15435$ & 3 & $5 / 2$ & $11 / 2$ & 3 & $5 / 2$ & $9 / 2$ & $-392 / 495$ \\
\hline 2 & $5 / 2$ & $7 / 2$ & 2 & $5 / 2$ & $9 / 2$ & $44 / 315$ & 3 & $5 / 2$ & $11 / 2$ & 3 & $5 / 2$ & $11 / 2$ & $13000 / 297$ \\
\hline 2 & $5 / 2$ & $9 / 2$ & 2 & $5 / 2$ & $7 / 2$ & $-44 / 315$ & 1 & $3 / 2$ & $1 / 2$ & 1 & $1 / 2$ & $3 / 2$ & $3 / 5$ \\
\hline 2 & $5 / 2$ & $9 / 2$ & 2 & $5 / 2$ & $9 / 2$ & $1681 / 99$ & 1 & $3 / 2$ & $5 / 2$ & 1 & $1 / 2$ & $3 / 2$ & $-21 / 25$ \\
\hline 2 & $5 / 2$ & $9 / 2$ & 2 & $5 / 2$ & $11 / 2$ & $72 / 55$ & 2 & $5 / 2$ & $3 / 2$ & 2 & $3 / 2$ & $1 / 2$ & $3 / 5$ \\
\hline 2 & $5 / 2$ & $11 / 2$ & 2 & $5 / 2$ & $9 / 2$ & $-72 / 55$ & 2 & $5 / 2$ & $3 / 2$ & 2 & $3 / 2$ & $5 / 2$ & $3 / 7$ \\
\hline 2 & $5 / 2$ & $11 / 2$ & 2 & $5 / 2$ & $11 / 2$ & $1248 / 55$ & 2 & $5 / 2$ & $5 / 2$ & 2 & $3 / 2$ & $5 / 2$ & $-288 / 343$ \\
\hline 1 & $1 / 2$ & $3 / 2$ & 1 & $1 / 2$ & $3 / 2$ & $24 / 25$ & 2 & $5 / 2$ & $5 / 2$ & 2 & $3 / 2$ & $7 / 2$ & $-1521 / 1715$ \\
\hline 2 & $3 / 2$ & $1 / 2$ & 2 & $3 / 2$ & $1 / 2$ & $3 / 5$ & 2 & $5 / 2$ & $7 / 2$ & 2 & $3 / 2$ & $5 / 2$ & $-216 / 343$ \\
\hline 2 & $3 / 2$ & $5 / 2$ & 2 & $3 / 2$ & $5 / 2$ & $1875 / 343$ & 2 & $5 / 2$ & $7 / 2$ & 2 & $3 / 2$ & $7 / 2$ & $64 / 343$ \\
\hline 2 & $3 / 2$ & $5 / 2$ & 2 & $3 / 2$ & $7 / 2$ & $120 / 343$ & 2 & $5 / 2$ & $9 / 2$ & 2 & $3 / 2$ & $7 / 2$ & $-11 / 7$ \\
\hline 2 & $3 / 2$ & $7 / 2$ & 2 & $3 / 2$ & $5 / 2$ & $-120 / 343$ & & & & & & & \\
\hline
\end{tabular}


Table D.5

Angular reduced matrix elements $\left\langle J^{\prime}\left\|\left[\alpha \times i(\alpha \times \tilde{\pi})^{(1)}\right]^{(1)}\right\| J\right\rangle$ between Spin(5) coupled states, for $\tau_{1} \leq 5 / 2$. A square root sign is to be understood over the magnitude of each matrix element.

\begin{tabular}{|c|c|c|c|c|c|c|c|c|c|c|c|c|c|}
\hline$\tau$ & $\tau_{1}$ & $J$ & $\tau^{\prime}$ & $\tau_{1}^{\prime}$ & $J^{\prime}$ & RME & $\tau$ & $\tau_{1}$ & $J$ & $\tau^{\prime}$ & $\tau_{1}^{\prime}$ & $J^{\prime}$ & $\mathrm{RME}$ \\
\hline 1 & $1 / 2$ & $3 / 2$ & 0 & $1 / 2$ & $3 / 2$ & 0 & 1 & $3 / 2$ & $1 / 2$ & 0 & $1 / 2$ & $3 / 2$ & 0 \\
\hline 2 & $3 / 2$ & $1 / 2$ & 1 & $3 / 2$ & $1 / 2$ & $-3 / 50$ & 1 & $3 / 2$ & $5 / 2$ & 0 & $1 / 2$ & $3 / 2$ & 0 \\
\hline 2 & $3 / 2$ & $5 / 2$ & 1 & $3 / 2$ & $5 / 2$ & $363 / 3430$ & 2 & $5 / 2$ & $3 / 2$ & 1 & $3 / 2$ & $1 / 2$ & $-3 / 50$ \\
\hline 2 & $3 / 2$ & $5 / 2$ & 1 & $3 / 2$ & $7 / 2$ & $12 / 343$ & 2 & $5 / 2$ & $3 / 2$ & 1 & $3 / 2$ & $5 / 2$ & $21 / 250$ \\
\hline 2 & $3 / 2$ & $7 / 2$ & 1 & $3 / 2$ & $5 / 2$ & $48 / 8575$ & 2 & $5 / 2$ & $5 / 2$ & 1 & $3 / 2$ & $5 / 2$ & $4356 / 42875$ \\
\hline 2 & $3 / 2$ & $7 / 2$ & 1 & $3 / 2$ & $7 / 2$ & $-288 / 8575$ & 2 & $5 / 2$ & $5 / 2$ & 1 & $3 / 2$ & $7 / 2$ & $288 / 8575$ \\
\hline 3 & $5 / 2$ & $3 / 2$ & 2 & $5 / 2$ & $3 / 2$ & $16 / 375$ & 2 & $5 / 2$ & $7 / 2$ & 1 & $3 / 2$ & $5 / 2$ & $-108 / 1715$ \\
\hline 3 & $5 / 2$ & $3 / 2$ & 2 & $5 / 2$ & $5 / 2$ & $-36 / 875$ & 2 & $5 / 2$ & $7 / 2$ & 1 & $3 / 2$ & $7 / 2$ & $648 / 1715$ \\
\hline 3 & $5 / 2$ & $5 / 2$ & 2 & $5 / 2$ & $3 / 2$ & $112 / 1125$ & 2 & $5 / 2$ & $9 / 2$ & 1 & $3 / 2$ & $7 / 2$ & 0 \\
\hline 3 & $5 / 2$ & $5 / 2$ & 2 & $5 / 2$ & $5 / 2$ & $-146689 / 2315250$ & 2 & $3 / 2$ & $1 / 2$ & 1 & $1 / 2$ & $3 / 2$ & $-3 / 50$ \\
\hline 3 & $5 / 2$ & $5 / 2$ & 2 & $5 / 2$ & $7 / 2$ & $512 / 15435$ & 2 & $3 / 2$ & $5 / 2$ & 1 & $1 / 2$ & $3 / 2$ & $-3 / 70$ \\
\hline 3 & $5 / 2$ & $7 / 2$ & 2 & $5 / 2$ & $5 / 2$ & $-18 / 1715$ & 3 & $5 / 2$ & $3 / 2$ & 2 & $3 / 2$ & $1 / 2$ & $-8 / 75$ \\
\hline 3 & $5 / 2$ & $7 / 2$ & 2 & $5 / 2$ & $7 / 2$ & $40328 / 6251175$ & 3 & $5 / 2$ & $3 / 2$ & 2 & $3 / 2$ & $5 / 2$ & $8 / 105$ \\
\hline 3 & $5 / 2$ & $7 / 2$ & 2 & $5 / 2$ & $9 / 2$ & $-154 / 18225$ & 3 & $5 / 2$ & $5 / 2$ & 2 & $3 / 2$ & $5 / 2$ & $3844 / 15435$ \\
\hline 3 & $5 / 2$ & $9 / 2$ & 2 & $5 / 2$ & $7 / 2$ & $5632 / 127575$ & 3 & $5 / 2$ & $5 / 2$ & 2 & $3 / 2$ & $7 / 2$ & $36481 / 154350$ \\
\hline 3 & $5 / 2$ & $9 / 2$ & 2 & $5 / 2$ & $9 / 2$ & $16129 / 80190$ & 3 & $5 / 2$ & $7 / 2$ & 2 & $3 / 2$ & $5 / 2$ & $-1024 / 5145$ \\
\hline 3 & $5 / 2$ & $9 / 2$ & 2 & $5 / 2$ & $11 / 2$ & $36 / 275$ & 3 & $5 / 2$ & $7 / 2$ & 2 & $3 / 2$ & $7 / 2$ & $95048 / 138915$ \\
\hline 3 & $5 / 2$ & $11 / 2$ & 2 & $5 / 2$ & $9 / 2$ & $16 / 2475$ & 3 & $5 / 2$ & $9 / 2$ & 2 & $3 / 2$ & $7 / 2$ & $-55 / 1134$ \\
\hline 3 & $5 / 2$ & $11 / 2$ & 2 & $5 / 2$ & $11 / 2$ & $-832 / 7425$ & & & & & & & \\
\hline
\end{tabular}

Table D.6

Angular reduced matrix elements $\left\langle J^{\prime}\left\|\left(a^{\dagger} \times \tilde{a}\right)^{(1)}\right\| J\right\rangle$ between Spin(5) coupled states, for $\tau_{1} \leq 5 / 2$. For reduced matrix elements of $\hat{j}$, multiply by $-\sqrt{5}$. A square root sign is to be understood over the magnitude of each matrix element.

\begin{tabular}{|c|c|c|c|c|c|c|c|c|c|c|c|c|c|}
\hline$\tau$ & $\tau_{1}$ & $J$ & $\overline{\tau^{\prime}}$ & $\overline{\tau_{1}^{\prime}}$ & $J^{\prime}$ & RME & $\bar{\tau}$ & $\overline{\tau_{1}}$ & $J$ & $\overline{\tau^{\prime}}$ & $\tau_{1}^{\prime}$ & $J^{\prime}$ & RME \\
\hline 0 & $1 / 2$ & $3 / 2$ & 0 & $1 / 2$ & $3 / 2$ & -3 & 2 & $3 / 2$ & $7 / 2$ & 2 & $3 / 2$ & $7 / 2$ & $-18 / 1715$ \\
\hline 1 & $3 / 2$ & $1 / 2$ & 1 & $3 / 2$ & $1 / 2$ & $3 / 10$ & 3 & $5 / 2$ & $3 / 2$ & 3 & $5 / 2$ & $3 / 2$ & $1 / 75$ \\
\hline 1 & $3 / 2$ & $5 / 2$ & 1 & $3 / 2$ & $5 / 2$ & $-507 / 350$ & 3 & $5 / 2$ & $3 / 2$ & 3 & $5 / 2$ & $5 / 2$ & $112 / 225$ \\
\hline 1 & $3 / 2$ & $5 / 2$ & 1 & $3 / 2$ & $7 / 2$ & $48 / 35$ & 3 & $5 / 2$ & $5 / 2$ & 3 & $5 / 2$ & $3 / 2$ & $-112 / 225$ \\
\hline 1 & $3 / 2$ & $7 / 2$ & 1 & $3 / 2$ & $5 / 2$ & $-48 / 35$ & 3 & $5 / 2$ & $5 / 2$ & 3 & $5 / 2$ & $5 / 2$ & $6241 / 9450$ \\
\hline 1 & $3 / 2$ & $7 / 2$ & 1 & $3 / 2$ & $7 / 2$ & $-162 / 35$ & 3 & $5 / 2$ & $5 / 2$ & 3 & $5 / 2$ & $7 / 2$ & $8 / 63$ \\
\hline 2 & $5 / 2$ & $3 / 2$ & 2 & $5 / 2$ & $3 / 2$ & $-3 / 25$ & 3 & $5 / 2$ & $7 / 2$ & 3 & $5 / 2$ & $5 / 2$ & $-8 / 63$ \\
\hline 2 & $5 / 2$ & $3 / 2$ & 2 & $5 / 2$ & $5 / 2$ & $144 / 175$ & 3 & $5 / 2$ & $7 / 2$ & 3 & $5 / 2$ & $7 / 2$ & $-4418 / 25515$ \\
\hline 2 & $5 / 2$ & $5 / 2$ & 2 & $5 / 2$ & $3 / 2$ & $-144 / 175$ & 3 & $5 / 2$ & $7 / 2$ & 3 & $5 / 2$ & $9 / 2$ & $616 / 3645$ \\
\hline 2 & $5 / 2$ & $5 / 2$ & 2 & $5 / 2$ & $5 / 2$ & $243 / 17150$ & 3 & $5 / 2$ & $9 / 2$ & 3 & $5 / 2$ & $7 / 2$ & $-616 / 3645$ \\
\hline 2 & $5 / 2$ & $5 / 2$ & 2 & $5 / 2$ & $7 / 2$ & $72 / 343$ & 3 & $5 / 2$ & $9 / 2$ & 3 & $5 / 2$ & $9 / 2$ & $6241 / 16038$ \\
\hline 2 & $5 / 2$ & $7 / 2$ & 2 & $5 / 2$ & $5 / 2$ & $-72 / 343$ & 3 & $5 / 2$ & $9 / 2$ & 3 & $5 / 2$ & $11 / 2$ & $784 / 495$ \\
\hline 2 & $5 / 2$ & $7 / 2$ & 2 & $5 / 2$ & $7 / 2$ & $-59858 / 15435$ & 3 & $5 / 2$ & $11 / 2$ & 3 & $5 / 2$ & $9 / 2$ & $-784 / 495$ \\
\hline 2 & $5 / 2$ & $7 / 2$ & 2 & $5 / 2$ & $9 / 2$ & $88 / 315$ & 3 & $5 / 2$ & $11 / 2$ & 3 & $5 / 2$ & $11 / 2$ & $13 / 1485$ \\
\hline 2 & $5 / 2$ & $9 / 2$ & 2 & $5 / 2$ & $7 / 2$ & $-88 / 315$ & 1 & $3 / 2$ & $1 / 2$ & 1 & $1 / 2$ & $3 / 2$ & $6 / 5$ \\
\hline 2 & $5 / 2$ & $9 / 2$ & 2 & $5 / 2$ & $9 / 2$ & $-289 / 198$ & 1 & $3 / 2$ & $5 / 2$ & 1 & $1 / 2$ & $3 / 2$ & $-42 / 25$ \\
\hline 2 & $5 / 2$ & $9 / 2$ & 2 & $5 / 2$ & $11 / 2$ & $144 / 55$ & 2 & $5 / 2$ & $3 / 2$ & 2 & $3 / 2$ & $1 / 2$ & $6 / 5$ \\
\hline 2 & $5 / 2$ & $11 / 2$ & 2 & $5 / 2$ & $9 / 2$ & $-144 / 55$ & 2 & $5 / 2$ & $3 / 2$ & 2 & $3 / 2$ & $5 / 2$ & $6 / 7$ \\
\hline 2 & $5 / 2$ & $11 / 2$ & 2 & $5 / 2$ & $11 / 2$ & $-351 / 55$ & 2 & $5 / 2$ & $5 / 2$ & 2 & $3 / 2$ & $5 / 2$ & $-576 / 343$ \\
\hline 1 & $1 / 2$ & $3 / 2$ & 1 & $1 / 2$ & $3 / 2$ & $-3 / 25$ & 2 & $5 / 2$ & $5 / 2$ & 2 & $3 / 2$ & $7 / 2$ & $-3042 / 1715$ \\
\hline 2 & $3 / 2$ & $1 / 2$ & 2 & $3 / 2$ & $1 / 2$ & $3 / 10$ & 2 & $5 / 2$ & $7 / 2$ & 2 & $3 / 2$ & $5 / 2$ & $-432 / 343$ \\
\hline 2 & $3 / 2$ & $5 / 2$ & 2 & $3 / 2$ & $5 / 2$ & $3 / 686$ & 2 & $5 / 2$ & $7 / 2$ & 2 & $3 / 2$ & $7 / 2$ & $128 / 343$ \\
\hline 2 & $3 / 2$ & $5 / 2$ & 2 & $3 / 2$ & $7 / 2$ & $240 / 343$ & 2 & $5 / 2$ & $9 / 2$ & 2 & $3 / 2$ & $7 / 2$ & $-22 / 7$ \\
\hline 2 & $3 / 2$ & $7 / 2$ & 2 & $3 / 2$ & $5 / 2$ & $-240 / 343$ & & & & & & & \\
\hline
\end{tabular}




\section{References}

[1] F. Iachello, in Group Theoretical Methods in Physics, edited by W. Beiglböck, A. Bohm, and E. Takasugi (Springer-Verlag, Berlin, 1979), Lecture Notes in Physics Vol. 94, p. 420.

[2] F. Iachello, Lie Algebras and Applications, Lecture Notes in Physics Vol. 708 (Springer, Berlin, 2006).

[3] F. Iachello and A. Arima, The Interacting Boson Model (Cambridge University Press, Cambridge, 1987).

[4] A. Bohr, Mat. Fys. Medd. Dan. Vid. Selsk. 26 (14) (1952).

[5] A. Bohr and B. R. Mottelson, Nuclear Deformations, Nuclear Structure Vol. 2 (World Scientific, Singapore, 1998).

[6] J. M. Eisenberg and W. Greiner, Nuclear Models: Collective and Single-Particle Phenomena, 3rd ed., Nuclear Theory Vol. 1 (North-Holland, Amsterdam, 1987).

[7] J. N. Ginocchio and M. W. Kirson, Phys. Rev. Lett. 44 (1980) 1744.

[8] A. E. L. Dieperink, O. Scholten, and F. Iachello, Phys. Rev. Lett. 44 (1980) 1747 .

[9] A. Bohr and B. R. Mottelson, Physica Scripta 22 (1980) 468.

[10] O. Scholten, F. Iachello, and A. Arima, Ann. Phys. (N.Y.) 115 (1978) 325.

[11] R. F. Casten, M. Wilhelm, E. Radermacher, N. V. Zamfir, and P. von Brentano, Phys. Rev. C 57 (1998) R1553.

[12] D. H. Feng, R. Gilmore, and S. R. Deans, Phys. Rev. C 23 (1981) 1254.

[13] F. Iachello, Phys. Rev. Lett. 85 (2000) 3580.

[14] L. Wilets and M. Jean, Phys. Rev. 102 (1956) 788.

[15] D. J. Rowe, Nucl. Phys. A 735 (2004) 372.

[16] D. J. Rowe, P. S. Turner, and J. Repka, J. Math. Phys. 45 (2004) 2761.

[17] F. Iachello, Phys. Rev. Lett. 44 (1980) 772.

[18] F. Iachello and P. Van Isacker, The Interacting Boson-Fermion Model (Cambridge University Press, Cambridge, 1991).

[19] A. B. Balantekin, I. Bars, and F. Iachello, Phys. Rev. Lett. 47 (1981) 19.

[20] F. Iachello, Phys. Rev. Lett. 95 (2005) 052503.

[21] R. F. Casten and N. V. Zamfir, Phys. Rev. Lett. 85 (2000) 3584.

[22] J. M. Arias, Phys. Rev. C 63 (2001) 034308. 
[23] A. Frank, C. E. Alonso, and J. M. Arias, Phys. Rev. C 65 (2001) 014301.

[24] Jing-Ye Zhang, N. V. Zamfir, R. F. Casten, and M. A. Caprio, Phys. Rev. C 64 (2001) 017302.

[25] N. V. Zamfir, M. A. Caprio, R. F. Casten, C. J. Barton, C. W. Beausang, Z. Berant, D. S. Brenner, W. T. Chou, J. R. Cooper, A. A. Hecht, R. Krücken, H. Newman, J. R. Novak, N. Pietralla, A. Wolf, and K. E. Zyromski, Phys. Rev. C 65 (2002) 044325.

[26] Da-li Zhang and Yu-xin Liu, Phys. Rev. C 65 (2002) 057301.

[27] J. M. Arias, C. E. Alonso, A. Vitturi, J. E. García-Ramos, J. Dukelsky, and A. Frank, Phys. Rev. C 68 (2003) 041302(R).

[28] D. Bonatsos, D. Lenis, N. Minkov, P. P. Raychev, and P. A. Terziev, Phys. Rev. C 69 (2004) 044316.

[29] N. Mărginean, S. M. Lenzi, A. Gadea, E. Farnea, S. J. Freeman, D. R. Napoli, D. Bazzacco, S. Beghini, B. R. Behera, P. G. Bizzeti, A. Bizzeti-Sona, D. Bucurescu, R. Chapman, L. Corradi, A. N. Deacon, G. de Angelis, F. Della Vedova, E. Fioretto, M. Ionescu-Bujor, A. Iordachescu, Th. Kröll, A. Latina, X. Liang, S. Lunardi, G. Montagnoli, R. Mărginean, M. Nespolo, G. Pollarolo, C. Rusu, F. Scarlassara, J. F. Smith, K. Spohr, A. M. Stefanini, S. Szilner, M. Trotta, C. A. Ur, B. J. Varley, and W. Zhimin, Phys. Lett. B 633 (2006) 696.

[30] R. Fossion, D. Bonatsos, and G. A. Lalazissis, Phys. Rev. C 73 (2006) 044310.

[31] C. E. Alonso, J. M. Arias, L. Fortunato, and A. Vitturi, Phys. Rev. C 72 (2005) 061302(R).

[32] M. S. Fetea, R. B. Cakirli, R. F. Casten, D. D. Warner, E. A. McCutchan, D. A. Meyer, A. Heinz, H. Ai, G. Gürdal, J. Qian, and R. Winkler, Phys. Rev. C 73 (2006) 051301(R).

[33] A. de-Shalit and I. Talmi, Nuclear Shell Theory, Pure and Applied Physics No. 14 (Academic, New York, 1963).

[34] A. Arima and F. Iachello, Ann. Phys. (N.Y.) 99 (1976) 253.

[35] G. Rakavy, Nucl. Phys. 4 (1957) 289.

[36] D. R. Bès, Nucl. Phys. 10 (1959) 373.

[37] S. Flügge, Practical Quantum Mechanics I, Grundlehren der mathematischen Wissenschaften Vol. 177 (Springer-Verlag, Berlin, 1971).

[38] J. P. Elliott, J. A. Evans, and P. Park, Phys. Lett. B 169 (1986) 309.

[39] M. Abramowitz and I. A. Stegun, Handbook of Mathematical Functions (Dover, New York, 1965).

[40] I. S. Gradshteyn and I. M. Ryzhik, Table of Integrals, Series, and Products, 5th ed. (Academic Press, Boston, 1994). 
[41] N. Kemmer, D. L. Pursey, and S. A. Williams, J. Math. Phys. 9 (1968) 1224.

[42] A. Arima and F. Iachello, Ann. Phys. (N.Y.) 123 (1979) 468.

[43] The multiplicity index $\tilde{\nu}_{\triangle}$ used here (e.g., Ref. [3]) can easily be interconverted with the index $\alpha$ of Ref. [16]. In either case, a non-orthogonal basis is first constructed, of angular functions characterized by the number of zero-coupled triplets $\nu_{\triangle}$ of the quadrupole tensor $\alpha$, and Gram-Schmidt orthogonalization is then carried out. After orthogonalization, $\nu_{\triangle}$ is no longer a good quantum number. The multiplicity index $\tilde{\nu}_{\triangle}$ is obtained by simply retaining the original $\nu \triangle$ values as labels, while $\alpha$ is obtained by sequential renumbering $(\alpha=1,2, \ldots)$.

[44] F. Iachello, Contemp. Math. 160 (1994) 151.

[45] Jin-Quan Chen, Group Representation Theory for Physicists (World Scientific, Singapore, 1989).

[46] W. Miller, Jr., Lie Theory and Special Functions (Academic Press, New York, 1968), Chap. 3.

[47] B. G. Wybourne, Classical Groups for Physicists (Wiley, New York, 1974).

[48] S. Kais and R. D. Levine, Phys. Rev. A 34 (1986) 4615.

[49] J.-P. Antoine, J.-P. Gazeau, P. Monceau, J. R. Klauder, and K. A. Penson, J. Math. Phys. 42 (2001) 2349.

[50] R. Lemus and A. Frank, J. Phys. A 26 (2003) 4901.

[51] T. Otsuka, A. Arima, and F. Iachello, Nucl. Phys. A 309 (1978) 1.

[52] D. D. Warner and R. F. Casten, Phys. Rev. C 28 (1983) 1798.

[53] M. A. Caprio, Phys. Rev. C 72 (2005) 054323.

[54] A. R. Edmonds, Angular Momentum in Quantum Mechanics, 2nd ed., Investigations in Physics No. 4 (Princeton University Press, Princeton, New Jersey, 1960).

[55] V. Maruhn-Rezwani, W. Greiner, and J. A. Maruhn, Phys. Lett. B 57 (1975) 109.

[56] The present definition (2.27) differs from that of Ref. [6] by a phase factor $i$. This makes the matrix elements of $T^{(M 1)}$ real, for simplicity of notation and consistency with Ref. [3].

[57] J. L. Wood, E. F. Zganjar, C. de Coster, and K. Heyde, Nucl. Phys. A 651 (1999) 323.

[58] Y. Khazov, A. A. Rodionov, S. Sakharov, and B. Singh, Nucl. Data Sheets 104 (2005) 497.

[59] A. A. Sonzogni, Nucl. Data Sheets 103 (2004) 1.

[60] M. A. Caprio, Phys. Rev. C 65 (2002) 031304(R). 
[61] M. A. Caprio, Phys. Rev. C 69 (2004) 044307.

[62] M. A. Caprio, Ph.D. thesis, Yale University (2003), nucl-ex/0502004.

[63] B. H. Flowers, Proc. R. Soc. London A 212 (1952) 248.

[64] B. F. Bayman and L. Silverberg, Nucl. Phys. 16 (1960) 625.

[65] S. Kuyucak, Ph.D. thesis, Yale University (1982).

[66] F. Iachello and S. Kuyucak, Ann. Phys. (N.Y.) 136 (1981) 19.

[67] P. Van Isacker, J. Math. Phys. 28 (1987) 957.

[68] R. Bijker and V. K. B. Kota, Ann. Phys. (N.Y.) 156 (1984) 110.

[69] C. E. Alonso, J. M. Arias, and A. Vitturi (in preparation).

[70] A. B. Balantekin, O. Castaños, and M. Moshinsky, Phys. Lett. B 284 (1992) 1.

[71] F. Iachello, Phys. Rev. Lett. 87 (2001) 052502.

[72] R. M. Clark, A. O. Macchiavelli, L. Fortunato, and R. Krücken, Phys. Rev. Lett. 96 (2006) 032501.

[73] F. Iachello, F. Pérez-Bernal, and P. H. Vaccaro, Chem. Phys. Lett. 375 (2003) 309.

[74] F. Pérez-Bernal (in preparation). 